\title{
Wa Ve phenomena

\section{Discrete diffraction managed solitons: threshold phenomena and rapid decay for general nonlinearities}

Mi-Ran Choi, Dirk Hundertmark, Young-Ran Lee

CRC Preprint 2016/20, September 2016

\section{KARLSRUHE INSTITUTE OF TECHNOLOGY}

\section{CRC 1173}

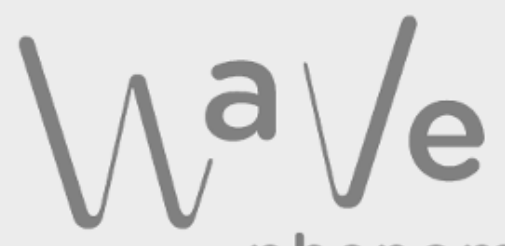

phenomena 


\section{Participating universities}

EBERHARD KARLS

UNIVERSITATT TUBINGEN

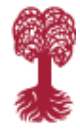

Funded by

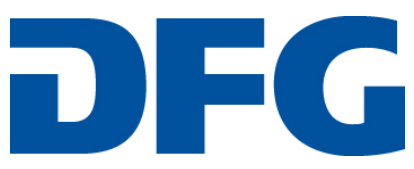

ISSN 2365-662X 


\title{
DISCRETE DIFFRACTION MANAGED SOLITONS: THRESHOLD PHENOMENA AND RAPID DECAY FOR GENERAL NONLINEARITIES
}

\author{
MI-RAN CHOI, DIRK HUNDERTMARK, YOUNG-RAN LEE
}

\begin{abstract}
We prove a threshold phenomenon for the existence/non-existence of energy minimizing solitary solutions of the diffraction management equation for strictly positive and zero average diffraction. Our methods allow for a large class of nonlinearities, they are, for example, allowed to change sign, and the weakest possible condition, it only has to be locally integrable, on the local diffraction profile. The solutions are found as minimizers of a nonlinear and nonlocal variational problem which is translation invariant. There exists a critical threshold $\lambda_{\text {cr }}$ such that minimizers for this variational problem exist if their power is bigger than $\lambda_{\text {cr }}$ and no minimizers exist with power less than the critical threshold. We also give simple criteria for the finiteness and strict positivity of the critical threshold. Our proof of existence of minimizers is rather direct and avoids the use of Lions' concentration compactness argument.

Furthermore, we give precise quantitative lower bounds on the exponential decay rate of the diffraction management solitons, which confirm the physical heuristic prediction for the asymptotic decay rate. Moreover, for ground state solutions, these bounds give a quantitative lower bound for the divergence of the exponential decay rate in the limit of vanishing average diffraction. For zero average diffraction, we prove quantitative bounds which show that the solitons decay much faster than exponentially. Our results considerably extend and strengthen the results of [15] and [16].
\end{abstract}

\section{Contents}

1. Introduction 2

2. Nonlinear estimates 7

2.1. Fractional linear estimates 7

2.2. Splitting the nonlocal nonlinearity 8

3. The existence proof 10

4. Threshold phenomena 16

5. Exponential decay for positive average diffraction 23

5.1. Some exponential decay 23

5.2. Boosting the decay rate 27

6. Super-exponential decay for zero average diffraction 29

6.1. Some super-exponential decay 30

6.2. Boosting the (super-exponential) decay rate 34

Appendix A. Some useful bounds 35

Appendix B. Boundedness, negativity, and strict subadditivity of the energy 40

Appendix C. The discrete IMS localization formula 45

Appendix D. The connection with nonlinear optics 46

$\begin{array}{ll}\text { References } & 48\end{array}$

Date: September 19, 2016, version general-diffraction-8-1.tex.

(C) 2016 by the authors. Faithful reproduction of this article, in its entirety, by any means is permitted for non-commercial purposes. 


\section{INTRODUCTION}

We study the existence and properties of solutions of the diffraction managed non-linear discrete Schrödinger equation

$$
\omega \varphi(x)=-d_{\mathrm{av}}(\Delta \varphi)(x)-\int_{\mathbb{R}} T_{r}^{-1}\left[P\left(T_{r} \varphi(x)\right)\right] \mu(d r),
$$

on $l^{2}(\mathbb{Z})$, where $\mu$ is a finite measure with compact support and $\omega$ a constant. Here, $\Delta f(x)=$ $f(x+1)-2 f(x)+f(x-1)$ is the discrete Laplacian on $\mathbb{Z}, T_{r}=e^{i r \Delta}$ is the solution operator of the free discrete Schrödinger equation in one dimension, the average diffraction $d_{\mathrm{av}}$ is either positive or zero, and $P$ is the nonlinear term. Previously, either only very simple pure power nonlinearities $P$ together with simple measures $\mu$, which correspond to piecewise constant local diffraction profiles $d_{0}$, or the specific third order nonlinearity $P(z)=|z|^{2} z, z \in \mathbb{C}$ and general probability measures $\mu$ have been studied, see the discussion in Appendix D. We will extend this to a large class of nonlinearities.

Discrete nonlinear dispersive equations such as the discrete nonlinear Schrödinger equation (1.1) arise in the context of nonlinear optics [4, 5, 11, 21, 28], the study of dynamics of biological molecules [9, 10], localized modes in anharmonic crystal in condensed matter physics, $[6,26]$. Here the discrete models arise as phenomenological models or as tight binding approximations, see, for example, [19, 22].

In the application to nonlinear optics, which is our main motivation for studying solutions of (1.1), $d_{\mathrm{av}}$ is the average diffraction along an array of waveguides and $\mu$ will be a probability measure with compact support related to the local periodic diffraction $d_{0}$ along the waveguide. Since we can treat arbitrary probability measures $\mu$ with compact support, our results hold for any local diffraction profile $d_{0}$ which is locally integrable. In particular, $\mu=\delta_{0}$, the Dirac mass at zero, is allowed, so our results include the well-known discrete NLS. We will discuss this more thoroughly in Appendix D.

To get the weak formulation of (1.1), let $\langle f, g\rangle:=\sum_{x \in \mathbb{Z}} \overline{f(x)} g(x)$ be the usual scalar product in $l^{2}(\mathbb{Z})$ and take the scalar product of $(1.1)$ with $h \in l^{2}(\mathbb{Z})$ to see that since $-\langle\Delta \varphi, h\rangle=\left\langle D_{+} \varphi, D_{+} h\right\rangle$, where the forward difference operator $D_{+}$is defined by

$$
\left(D_{+} f\right)(x):=f(x+1)-f(x)
$$

for any $x \in \mathbb{Z}$ and using the unicity of $T_{r}$ one has

$$
\left\langle\int_{\mathbb{R}} T_{r}^{-1}\left[P\left(T_{r} \varphi\right)\right] \mu(d r), h\right\rangle=\int_{\mathbb{R}}\left\langle P\left(T_{r} \varphi\right), T_{r} h\right\rangle \mu(d r)
$$

and therefore the weak formulation of (1.1) is given by

$$
\omega\langle\varphi, h\rangle=d_{\mathrm{av}}\left\langle D_{+} \varphi, D_{+} h\right\rangle-\int_{\mathbb{R}}\left\langle P\left(T_{r} \varphi\right), T_{r} h\right\rangle \mu(d r)
$$

for all $h \in l^{2}(\mathbb{Z})$.

The diffraction management equation (1.1), or better, its weak form (1.2), has a variational structure. We assume that $P$ is an odd nonlinearity of the form

$$
P(z)=p(|z|) z
$$

for $z \in \mathbb{C}$. To use this, let $V$ be a differentiable function with $V^{\prime}(a)=P(a)$ for $a \geq 0$, for example,

$$
V(a)=\int_{0}^{a} P(s) d s \quad \text { for } a \geq 0
$$


Then the constrained minimization problem associated with (1.1) is given by

$$
E_{\lambda}^{d_{\mathrm{av}}}:=\inf \left\{H(\varphi): \varphi \in l^{2}(\mathbb{Z}),\|\varphi\|_{l^{2}(\mathbb{Z})}^{2}=\lambda\right\},
$$

where $\lambda>0$ and the Hamiltonian, or the energy, takes the form

$$
H(\varphi):=\frac{d_{\mathrm{av}}}{2}\left\|D_{+} \varphi\right\|_{l^{2}(\mathbb{Z})}^{2}-N(\varphi)
$$

with the nonlocal nonlinear 'potential'

$$
N(\varphi):=\int_{\mathbb{R}} \sum_{x \in \mathbb{Z}} V\left(\left|T_{r} \varphi(x)\right|\right) \mu(d r) .
$$

It turns out that any minimizer of (1.5), that is, any $\varphi \in l^{2}(\mathbb{Z})$ with $\|\varphi\|_{l^{2}(\mathbb{Z})}^{2}=\lambda$ such that $E_{\lambda}^{d_{\mathrm{av}}}=H(\varphi)$, will be a solution of corresponding Euler-Lagrange equation (1.1). Thus we are led to study the minimization problem (1.5) and to investigate the properties of its solution. An obstacle for the existence proof is the invariance of the Hamiltonian under shifts so the variational problem is invariant under a non-compact group. Hence there is a potential loss of compactness, since minimizing sequences can easily converge weakly to zero.

While it is possible to formulate conditions directly on the nonlinearity $P$ in (1.1), we find it more convenient to use conditions on the nonlinear potential $V$ related to it by (1.4). Our main assumptions on the nonlinear potential $V: \mathbb{R}_{+} \rightarrow \mathbb{R}$ are

A1) $V$ is continuous on $\mathbb{R}_{+}=[0, \infty)$ and differentiable on $(0, \infty)$ with $V(0)=0$. There exist $2<\gamma_{1} \leq \gamma_{2}<\infty$ such that

$$
\left|V^{\prime}(a)\right| \lesssim a^{\gamma_{1}-1}+a^{\gamma_{2}-1} \quad \text { for all } a>0 .
$$

A2) $V$ is continuous on $\mathbb{R}_{+}$and differentiable on $(0, \infty)$ with $V(0)=0$. There exists $\gamma_{0}>2$ such that

$$
V^{\prime}(a) a \geq \gamma_{0} V(a) \quad \text { for all } a>0 .
$$

A3) There exists $a_{0}>0$ such that $V\left(a_{0}\right)>0$.

The three assumptions above are our main requirements on the nonlinear potential. They are enough to prove a threshold phenomenon: solutions exist at least for large enough power $\lambda=\|\varphi\|_{2}^{2}$. In order to guarantee the existence of solutions for arbitrarily small $\lambda$, we need to strengthen assumption A3 to

A4) If $d_{\mathrm{av}}>0$ we assume that there exist $\varepsilon>0$ and $2 \leq \kappa<6$ such that

$$
V(a) \gtrsim a^{\kappa} \quad \text { for all } 0<a \leq \varepsilon .
$$

If $d_{\mathrm{av}}=0$ we assume that $V(a)>0$ for all $0<a \leq \varepsilon$.

Remarks 1.1. (i) An integration shows that A1 implies

$$
|V(a)| \lesssim a^{\gamma_{1}}+a^{\gamma_{2}}
$$

Much more important for us is the fact that A1 allows us to control the nonlocal nonlinearity $N$ under splitting, see Lemma 2.7 and the discussion in section 2.2.

(ii) Examples of nonlinearities obeying assumptions A1 through A3 are given by

$$
V(a)=\sum_{j=1}^{J} c_{j} a^{s_{j}}
$$


with $c_{j} \geq 0,2<s_{j}<\infty$, and $J \in \mathbb{N}$, but our assumptions also allow nonlinear potentials which can become negative, for example,

$$
V(a)=-a^{4}+a^{6} \quad \text { for } a \geq 0
$$

is allowed. It certainly fulfillls A1. Since

$$
V^{\prime}(a) a=-4 a^{4}+6 a^{6}=4\left(-a^{4}+a^{6}\right)+2 a^{6} \geq 4 V(a),
$$

it also obeys A2. Moreover, $V\left(a_{0}\right)>0$ for all large enough $a_{0}$, so A3 holds.

If we did not assume A3, then the nonlinearities could also be strictly negative for all $a>0$, for example, $V(a)=-a^{4}-a^{6}$ obeys $\mathbf{A} 1$ and because of

$$
V^{\prime}(a) a=-4 a^{4}-6 a^{6}=6\left(-\frac{4}{6} a^{4}-a^{6}\right) \geq 6 V(a)
$$

also A2, but then the critical threshold $\lambda_{\text {cr }}$ given in Theorem 4.1 would be infinite. The threshold is finite if and only if, for some $f \in l^{2}(\mathbb{Z})$ we have $N(f)>0$, see part (iv) of Theorem 4.1 below.

Concerning the existence and nonexistence of solutions, we have

Theorem 1.2 (Threshold phenomenon for existence/non-existence). Assume that $V$ obeys assumptions $\boldsymbol{A} 1$ through $\boldsymbol{A} 3$ and that $d_{\mathrm{av}} \geq 0$.

(i) There exists a threshold $0 \leq \lambda_{\mathrm{cr}}<\infty$ such that $E_{\lambda}^{d_{\mathrm{av}}}=0$ for $0 \leq \lambda \leq \lambda_{\mathrm{cr}}$ and $-\infty<E_{\lambda}^{d_{\mathrm{av}}}<0$ for $\lambda>\lambda_{\mathrm{cr}}$.

(ii) If $d_{\mathrm{av}}>0$ and $0<\lambda<\lambda_{\mathrm{cr}}$, then no minimizer for the constrained minimization problem (1.5) exists. If $\gamma_{1} \geq 6$, then $\lambda_{\mathrm{cr}}>0$.

(iii) If $d_{\mathrm{av}} \geq 0$ and $\lambda>\lambda_{\mathrm{cr}}$, then any minimizing sequence for (1.5) is up to translations relatively compact in $l^{2}(\mathbb{Z})$, in particular, there exists a minimizer for (1.5). This minimizer is also a solution of the diffraction management equation (1.1) for some Lagrange multiplier $\omega<2 E_{\lambda}^{d_{\mathrm{av}}} / \lambda<0$.

(iv) If $V$ obeys, in addition, $\boldsymbol{A} 4$, then $\lambda_{\mathrm{cr}}=0$.

Remarks 1.3. (i) The proof of Theorem 1.2 is given at the end of Section 4. The precise definition of the threshold $\lambda_{\text {cr }}$ is given in Definition 4.8. As we will see in Theorem 3.1, minimizing sequences for (1.5) are relatively compact in $l^{2}(\mathbb{Z})$ modulo translations if and only if $E_{\lambda}^{d_{\mathrm{av}}}<0$. So when $\lambda=\lambda_{\text {cr }}$ minimizers might exist, but minimizing sequences do not have be be precompact modulo translations.

(ii) Using $h=\varphi$ in (1.2), it is clear that the Lagrange multipliers are

$$
\omega=\omega(\varphi)=\frac{d_{\mathrm{av}}\left\langle D_{+} \varphi, D_{+} \varphi\right\rangle-\int_{\mathbb{R}}\left\langle P\left(T_{r} \varphi\right), T_{r} \varphi\right\rangle \mu(d r)}{\langle\varphi, \varphi\rangle}
$$

and using assumption A2 this will yield a rather direct proof of $\omega(\varphi)<2 E_{\lambda}^{d_{\mathrm{av}}} / \lambda<0$ for all minimizers $\varphi$, see (3.15).

If $\varphi$ is a solution of (1.1), or rather of its weak version (1.2), one can ask how well it will be localized. As it turns out, the answer to this depends on whether $d_{\mathrm{av}}=0$ or $d_{\mathrm{av}}>0$. In an earlier paper [16], super-exponential decay of solutions for $d_{\mathrm{av}}=0$ was shown in the case that the nonlinearity is cubic, $P(a)=|a|^{2} a$ or $V(a)=\frac{1}{4}|a|^{4}$. The case of positive average diffraction was not studied.

There is a simple physical heuristic guess for decay rate of solutions of (1.2): Assume that $\varphi$ decays exponentially and make the ansatz $\varphi(x)=e^{-\nu x}$ for $x \gg 1$. Plugging this 
into (1.1) and hoping that, even despite possible nonlocal effects, the nonlinearity in (1.1) is of higher order than $e^{-\nu x}$, then

$$
\begin{aligned}
\omega e^{-\nu x} & =-d_{\mathrm{av}} \Delta\left(e^{-\nu \cdot}\right)(x)+o\left(e^{-\nu x}\right) \\
& =-d_{\mathrm{av}}\left(e^{-\nu(x+1)}-2 e^{-\nu x}+e^{-\nu(x-1)}\right)+o\left(e^{-\nu x}\right) \\
& =-2 d_{\mathrm{av}}(\cosh (\nu)-1) e^{-\nu x}+o\left(e^{-\nu x}\right) .
\end{aligned}
$$

Letting $x \rightarrow \infty$, one sees that this implies $\omega<0$ and $2 d_{\mathrm{av}}(\cosh (\nu)-1)=|\omega|$, or, with $\cosh ^{-1}$ the inverse function of $\cosh :[0, \infty) \rightarrow[1, \infty)$,

$$
\nu=\cosh ^{-1}\left(\frac{|\omega|}{2 d_{\mathrm{av}}}+1\right)
$$

which is a rather precise prediction for the exponential decay rate. A remarkable feature of it is that it predicts $\nu \rightarrow \infty$ if $d_{\mathrm{av}} \rightarrow 0$ as long as $\omega$ stays away from zero.

Of course, this all depends on in which sense the nonlocal nonlinear terms in (1.1) are really of lower exponential order. Nevertheless, this simple physical heuristic is not far from the truth, because of

Theorem 1.4 (Decay for positive average diffraction). Assume $d_{\mathrm{av}}>0$ and $V$ obeys assumption $\boldsymbol{A} 1$. Then any solution $\varphi$ of (1.1) with $\omega<0$ decays exponentially and the decay rate is given by the above heuristic in the sense that

$$
\nu_{*}(\varphi):=\sup \left\{\nu>0 \mid\left(x \mapsto e^{\nu|x|} \varphi(x)\right) \in l^{2}(\mathbb{Z})\right\} \geq \cosh ^{-1}\left(\frac{|\omega|}{2 d_{\mathrm{av}}}+1\right) .
$$

Remark 1.5. As we will see in Theorem 4.1 below, the ground state solutions, that is, the ones with minimal energy, are solutions with $\omega<2 E_{\lambda}^{d_{\mathrm{av}}} / \lambda<0$ for all $d_{\mathrm{av}}>0$. At the moment, we cannot rule out that there are solutions of (1.1) for which $\nu_{*}>\cosh ^{-1}\left(\frac{|\omega|}{2 d_{\mathrm{av}}}+1\right)$.

Given the lower bound on the exponential decay rate given in (1.11), one expects that $\nu_{*}\left(\varphi_{d_{\mathrm{av}}}\right) \rightarrow \infty$ as $d_{\mathrm{av}} \rightarrow 0$, as long as the corresponding Lagrange multipliers $\omega=\omega\left(\varphi_{d_{\mathrm{av}}}\right)$ stay away from zero. In general, this might not be the case, but it is true for ground state solutions.

Corollary 1.6. Let $\lambda>0, d_{\mathrm{av}}>0$, and $\mathcal{M}_{\lambda}^{d_{\mathrm{av}}}$ the set of minimizers of the constrained minimization problem (1.5). Then for fixed $\lambda>0$ and any choice $\varphi_{d_{\mathrm{av}}} \in \mathcal{M}_{\lambda}^{d_{\mathrm{av}}}$ the exponential decay rates diverge in the limit of small average dispersion. More precisely, we have the lower bound

$$
\liminf _{d_{\mathrm{av}} \rightarrow 0} \frac{\nu_{*}\left(\varphi_{d_{\mathrm{av}}}\right)}{\cosh ^{-1}\left(\frac{\left|E_{\lambda}^{0}\right|-\delta}{\lambda d_{\mathrm{av}}}+1\right)} \geq 1
$$

for any $0<\delta<\left|E_{\lambda}^{0}\right|$, so the exponential decay rate $\nu_{*}\left(\varphi_{d_{\mathrm{av}}}\right)$ diverges at least logarithmically as $d_{\mathrm{av}} \rightarrow 0$.

Proof. This is, in fact, a simple consequence of the lower bound (1.11), the negativity of $E_{\lambda}^{d_{\mathrm{av}}}$, guaranteed by Theorem 3.1, its monotonicity ${ }^{1}$ in $d_{\mathrm{av}} \geq 0$, and the bound on the Lagrange multipliers from Theorem 3.1, which imply that for all $\delta>0$ one has $\left|\omega\left(\varphi_{d_{\mathrm{av}}}\right)\right| \lambda \geq$ $2\left|E_{\lambda}^{d_{\mathrm{av}}}\right| \geq 2\left(\left|E_{\lambda}^{0}\right|-\delta\right)$ for all small enough $d_{\mathrm{av}}>0$.

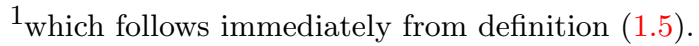


Given that the exponential decay rate of the ground states for average diffraction $d_{\mathrm{av}}>0$ diverges as $d_{\mathrm{av}} \rightarrow 0$, one can ask how fast solutions of (1.2) decay when $d_{\mathrm{av}}=0$. This was done in [16] for the special fourth order nonlinearity $V(a) \sim a^{4}$, but it holds in much greater generality.

Theorem 1.7 (Super-exponential decay for zero average diffraction). Assume $d_{\mathrm{av}}=0$ and $V$ obeys assumption $\boldsymbol{A} 1$. Then any solution $\varphi$ of (1.1) with $\omega \neq 0$ decays superexponentially, more precisely,

$$
\nu_{* *}(\varphi):=\sup \left\{\nu>0 \mid\left(x \mapsto(|x|+1)^{\nu(|x|+1)} \varphi(x)\right) \in l^{2}(\mathbb{Z})\right\} \geq \frac{2 \gamma_{1}-3}{2\left(\gamma_{1}-1\right)} .
$$

Remark 1.8. For $\gamma_{1}=4$, this yields the lower bound $\nu_{* *}(\varphi) \geq 5 / 6$ which is much better than the lower bound $\nu_{* *}(\varphi) \geq 1 / 4$ proven in [16].

Our paper is organized as follows: In Section 2 we develop the main tools needed for the existence proof. This includes new fractional linear bounds on the building blocks from Definition 2.4, which are needed to control the nonlocal nonlinearity $N(f)$ under splitting. That minimizing sequences for (1.5) are precompact modulo translations, that is, there exist suitable translations such that the translated minimizing sequence has a strongly convergent subsequence, if and only if $E_{\lambda}^{d_{\mathrm{av}}}<0$ is the content of Theorem 3.1. Our proof in Section 3 is based on non-splitting bounds for minimizing sequences given in Propositions 3.2 and 3.4, which together with a simple characterization of strong convergence in $l^{2}(\mathbb{Z})$ given in Lemma 3.8 imply precompactness of minimizing sequences modulo translations once $E_{\lambda}^{d_{\mathrm{av}}}<0$. This is similar, at least in spirit, to our companion paper [7] for the continuous case.

The threshold phenomenon is then studied in Section 4 and the proof of Theorem 1.2 is given at the end of this section. It turns out that Assumptions $\mathbf{A} 1$ and $\mathbf{A} 2$ are enough to yield a threshold phenomenon, see Theorem 4.1, but it could happen that $\lambda_{\text {cr }}$ is infinite, in which case no minimizers of 1.5 exist for any $\lambda>0$. Assumption A3 is used only to guarantee the finiteness of the threshold and $\mathbf{A} 4$ guarantees that the threshold is zero.

Unlike the continuous case we are able to prove strong lower bounds on the exponential decay rate for positive average diffraction, which confirm the physical heuristic, and strong lower bounds on the super-exponential decay rate for vanishing average diffraction, which improve earlier bounds given in [16]. These bounds are established in a two-step process: First we prove some (super-) exponential decay, see Proposition 5.1 in Section 5.1, respectively Proposition 6.2 in Section 6.1, and then give arguments which allow us to boost the decay rate, see Proposition 5.11 in Section 5.2, respectively Proposition 6.5 in Section 6.2. These results are based on several intermediate results, in particular, we need suitable a-priori bounds on exponentially twisted versions of the building blocks from Definition 5.3 for the derivative of the nonlinearity $N$.

In Appendix A, we gather some useful bounds for the space time norms of solutions of the free discrete Schrödinger equation on $l^{2}(\mathbb{Z})$. These estimates have analogous results on $l^{2}\left(\mathbb{Z}^{d}\right)$, similar to the discussion in [16], for example, but we give them only for $l^{2}(\mathbb{Z})$ for brevity. Lemma A.1 looks somewhat technical, at first, but is at the heart of most of our results in this work.

In Appendix B, we give the somewhat technical proof of negativity and subadditvity of the ground state energy $E_{\lambda}^{d_{\mathrm{av}}}$ from (1.5). The proof of subadditivity is similar to the continuous case and given for the convenience of the reader, it also immediately yields strict subadditivity once $E_{\lambda}^{d_{\mathrm{av}}}<0$. That Assumption A4 implies $E_{\lambda}^{d_{\mathrm{av}}}<0$ for any $\lambda>0$ and all $d_{\mathrm{av}} \geq 0$ turns out to be very much different from the continuous case where Gaussians form a convenient set of initial conditions, since on $l^{2}(\mathbb{Z})$ there is no simple family of initial 
conditions for which one can explicitly compute the time evolution under the free discrete Schrödinger evolution.

Appendix C discusses a discrete version of the well-known ${ }^{2}$ IMS localization formula, which is needed for strictly positive average diffraction. Finally, in Appendix D, we give for the convenience of the reader a short discussion on how the highly nonlocal diffraction management equation (1.1) arises in the study of solitary solutions of diffraction managed waveguides arrays.

\section{NonlineAR ESTIMATES}

2.1. Fractional linear estimates. First, we gather some bounds which will be used in the proofs of Proposition 3.2 and Proposition 5.1, which are the basis for the proofs of

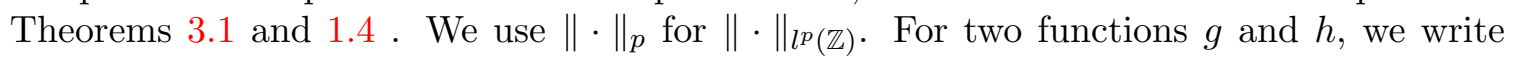
$g \lesssim h$ if there exists a constant $C>0$ such that $g \leq C h$.

The space $L^{p}(\mathbb{Z} \times \mathbb{R}, d x \mu(d r))$ consists of all space-time functions with finite norm

$$
\|f\|_{L^{p}(\mathbb{Z} \times \mathbb{R}, d x \mu(d r))}:= \begin{cases}\left(\int_{\mathbb{R}} \sum_{x \in \mathbb{Z}}|f(x, r)|^{p} \mu(d r)\right)^{1 / p} & \text { if } 1 \leq p<\infty, \\ \operatorname{esssup}_{r \in \operatorname{supp} \mu}\|f(\cdot, r)\|_{\infty} & \text { if } p=\infty\end{cases}
$$

where the essential supremum is with respect to the measure $\mu$, that is, modulo sets of $\mu$-measure zero.

A simple but useful bound is given in

Lemma 2.1. Let $1 \leq q \leq p \leq \infty$ and $f \in l^{q}(\mathbb{Z})$. Then

$$
\left\|T_{r} f\right\|_{L^{p}(\mathbb{Z} \times \mathbb{R}, d x \mu(d r))} \lesssim\|f\|_{q}
$$

where the implicit constant depends only on $q, p, \mu(\mathbb{R})$ and, if $q \neq 2$, also on $\operatorname{supp} \mu$.

Proof. Since $\|g\|_{p} \leq\|g\|_{q}$ for all $1 \leq q \leq p \leq \infty$, we get for $p<\infty$

$$
\int_{\mathbb{R}} \sum_{x \in \mathbb{Z}}\left|T_{r} f\right|^{p} \mu(d r)=\int_{\mathbb{R}}\left\|T_{r} f\right\|_{p}^{p} \mu(d r) \leq \int_{\mathbb{R}}\left\|T_{r} f\right\|_{q}^{p} \mu(d r) \leq \mu(\mathbb{R}) e^{4 B p|1-2 / q|}\|f\|_{q}^{p} .
$$

where we used (A.2) and chose $B>0$ such that $\operatorname{supp} \mu \subset[-B, B]$. If $p=\infty$,

$$
\left\|T_{r} f\right\|_{L^{\infty}(\mathbb{Z} \times \mathbb{R}, d x \mu(d r))} \leq \sup _{r \in[-B, B]}\left\|T_{r} f\right\|_{\infty} \leq \sup _{r \in[-B, B]}\left\|T_{r} f\right\|_{q} \leq e^{4 B|1-2 / q|}\|f\|_{q}
$$

Lemma 2.2 (Bilinear estimate). Let $1 \leq p \leq \infty, f_{1}, f_{2} \in l^{2}(\mathbb{Z})$, set $s=\operatorname{dist}\left(\operatorname{supp} f_{1}, \operatorname{supp} f_{2}\right)$, the distance of their supports, and $B>0$ such that $\operatorname{supp} \mu \subset[-B, B]$. Then

$$
\left\|T_{r} f_{1} T_{r} f_{2}\right\|_{L^{p}(\mathbb{Z} \times \mathbb{R}, d x \mu(d r))} \lesssim \min \left(1, \frac{8 e^{16 B}(4 B)^{\left\lceil\frac{s}{2}\right\rceil}}{\left\lceil\frac{s}{2}\right\rceil !}\right)\left\|f_{1}\right\|_{2}\left\|f_{2}\right\|_{2},
$$

where we used $\lceil s\rceil:=\min \{n \in \mathbb{Z} \mid s \leq n\}$ and the implicit constant depends only on $\mu(\mathbb{R})$ and $p$.

Proof. The proof of (2.1) is based on the strong bilinear estimate from Lemma A.1 in the appendix, which strengthens and simplifies the strong bilinear bound from [15, 16]. Choosing $B$ large enough so that $\operatorname{supp} \mu \subset[-B, B]$ and using (A.5) we get

$$
\left\|T_{r} f_{1} T_{r} f_{2}\right\|_{L^{p}(\mathbb{Z} \times \mathbb{R}, d x \mu(d r))}=\left(\int_{\mathbb{R}}\left\|T_{r} f_{1} T_{r} f_{2}\right\|_{p}^{p} \mu(d r)\right)^{1 / p} \leq \mu(\mathbb{R})^{1 / p} \sup _{r \in[-B, B]}\left\|T_{r} f_{1} T_{r} f_{2}\right\|_{p}
$$

\footnotetext{
${ }^{2}$ See, for example, Section 3.1 of [8] and references therein.
} 


$$
\lesssim \min \left(1, \frac{8 e^{16 B}(4 B)^{\left\lceil\frac{s}{2}\right\rceil}}{\left\lceil\frac{s}{2}\right\rceil !}\right)\left\|f_{1}\right\|_{2}\left\|f_{2}\right\|_{2}
$$

which proves (2.1).

Remark 2.3. Since $n ! \geq e^{n \ln n-n}$ and $(4 B)^{\left\lceil\frac{s}{2}\right\rceil} \lesssim e^{\frac{s}{2} \ln (4 B)}$, Lemma 2.2 implies the bounds

$$
\left\|T_{r} f_{1} T_{r} f_{2}\right\|_{L^{p}(\mathbb{Z} \times \mathbb{R}, d x \mu(d r))} \lesssim \min \left(1, s^{-\alpha s}\right)\left\|f_{1}\right\|_{2}\left\|f_{2}\right\|_{2}
$$

for all $0<\alpha<\frac{1}{2}$ and all $1 \leq p \leq \infty$. Here, if $s=0$, we set $0^{-\alpha 0}:=\lim _{s \rightarrow 0+} s^{-\alpha s}=1$.

The following will be the building blocks for our bounds on the nonlocal nonlinearity, their definition is motivated by the splitting of the nonlinear potential in Lemma 2.7.

Definition 2.4. For any $2 \leq \gamma<\infty$, let

$$
M_{\mu}^{\gamma}\left(f_{1}, f_{2}\right):=\int_{\mathbb{R}} \sum_{x \in \mathbb{Z}}\left|T_{r} f_{1}(x)\right|\left|T_{r} f_{2}(x)\right|\left(\left|T_{r} f_{1}(x)\right|+\left|T_{r} f_{2}(x)\right|\right)^{\gamma-2} \mu(d r) .
$$

Proposition 2.5. Let $s=\operatorname{dist}\left(\operatorname{supp} f_{1}, \operatorname{supp} f_{2}\right), 2 \leq \gamma<\infty$, and $0<\alpha<\frac{1}{2}$, then

$$
M_{\mu}^{\gamma}\left(f_{1}, f_{2}\right) \lesssim \min \left(1, s^{-\alpha s}\right)\left\|f_{1}\right\|_{2}\left\|f_{2}\right\|_{2}\left(\left\|f_{1}\right\|_{2}+\left\|f_{2}\right\|_{2}\right)^{\gamma-2}
$$

where the implicit constant depends only on $\operatorname{supp} \mu, \mu(\mathbb{R}), \gamma$, and $\alpha$.

Proof. Taking a supremum out of the integral we get

$$
\begin{aligned}
M_{\mu}^{\gamma}\left(f_{1}, f_{2}\right) & =\int_{\mathbb{R}} \sum_{x \in \mathbb{Z}}\left|T_{r} f_{1}(x) T_{r} f_{2}(x)\right|\left(\left|T_{r} f_{1}(x)\right|+\left|T_{r} f_{2}(x)\right|\right)^{\gamma-2} \mu(d r) \\
& \leq\left\|T_{r} f_{1} T_{r} f_{2}\right\|_{L^{1}(\mathbb{Z} \times \mathbb{R}, d x \mu(d r))}\left(\sup _{r \in \mathbb{R}}\left\|T_{r} f_{1}\right\|_{\infty}+\sup _{r \in \mathbb{R}}\left\|T_{r} f_{2}\right\|_{\infty}\right)^{\gamma-2} .
\end{aligned}
$$

Applying $\left\|T_{r} f\right\|_{\infty} \leq\left\|T_{r} f\right\|_{2}=\|f\|_{2}$ and (2.2) for the first factor yields (2.3).

\subsection{Splitting the nonlocal nonlinearity. Recall}

$$
N(f):=\int_{\mathbb{R}} \sum_{x \in \mathbb{Z}} V\left(\left|T_{r} f(x)\right|\right) \mu(d r) .
$$

The inequality (1.9) and Lemma 2.1 immediatley yield

Proposition 2.6 (Boundedness). Let $2 \leq \gamma_{1} \leq \gamma_{2}<\infty$. Then for all $f \in l^{2}(\mathbb{Z})$

$$
N(f) \lesssim\|f\|_{2}^{\gamma_{1}}+\|f\|_{2}^{\gamma_{2}}
$$

where the implicit constant depends only on $\mu(\mathbb{R})$.

Since $N(f)$ is highly nonlocal in $f$, it is difficult to control $N(f)$, when $f$ splits into $f=$ $f_{1}+f_{2}$ where $f_{1}$ and $f_{2}$ have widely separated supports. The following simple observation helps at this stage and is at the heart of all our estimates.

Lemma 2.7. Assume that $V$ obyes A1. Then

$$
\mid V(|z+w|)-V(|z|)-V(|w|))\left|\lesssim\left((|z|+|w|)^{\gamma_{1}-2}+(|z|+|w|)^{\gamma_{2}-2}\right)\right| z|| w \mid
$$

for all $z, w \in \mathbb{C}$. 
Proof. Since $V(0)=0$, we have $V(|z+w|)-V(|z|)-V(|w|)=0$ if at least one of $z$ and $w$ equals zero. So assume $z, w \neq 0$ in the following. Then

$$
\begin{aligned}
V(|z+w|)-V(|z|)-V(|w|)= & {\left[\frac{1}{|z|+|w|} V(|z+w|)-\frac{1}{|z|} V(|z|)\right]|z| } \\
& +\left[\frac{1}{|z|+|w|} V(|z+w|)-\frac{1}{|w|} V(|w|)\right]|w| .
\end{aligned}
$$

Moreover,

$$
\frac{1}{|z|+|w|} V(|z+w|)-\frac{1}{|z|} V(|z|)=\frac{1}{|z|+|w|}(V(|z+w|)-V(|z|))-\frac{|w|}{(|z|+|w|)|z|} V(|z|)
$$

Let $c=\min (|z|,|z+w|)$ and $d=\max (|z|,|z+w|) \leq|z|+|w|$. Then $d-c=|| z+w|-| z|| \leq|w|$ and using A1, we have

$$
\begin{aligned}
|V(|z+w|)-V(|z|)| & \leq \int_{c}^{d}\left|V^{\prime}(a)\right| d a \lesssim\left(d^{\gamma_{1}-1}+d^{\gamma_{2}-1}\right)(d-c) \\
& \leq\left((|z|+|w|)^{\gamma_{1}-1}+(|z|+|w|)^{\gamma_{2}-1}\right)|w|
\end{aligned}
$$

Since $V(0)=0$, A1 also implies

$$
|V(|z|)| \lesssim\left(|z|^{\gamma_{1}-1}+|z|^{\gamma_{2}-1}\right)|z|
$$

Using the two inequalities above in (2.6) shows

$$
\left|\frac{1}{|z|+|w|} V(|z+w|)-\frac{1}{|z|} V(|z|)\right| \lesssim\left((|z|+|w|)^{\gamma_{1}-2}+(|z|+|w|)^{\gamma_{2}-2}\right)|w|
$$

and a similar inequality holds when we interchange $z$ and $w$. Hence (2.5) implies (2.4).

The following is our main tool to control the nonlocal nonlinearity.

Proposition 2.8 (Splitting). Let $f_{1}, f_{2} \in l^{2}(\mathbb{Z})$ and $s=\operatorname{dist}\left(\operatorname{supp} f_{1}, \operatorname{supp} f_{2}\right)$. Then for all $2 \leq \gamma_{1} \leq \gamma_{2}<\infty$

$$
\left|N\left(f_{1}+f_{2}\right)-N\left(f_{1}\right)-N\left(f_{2}\right)\right| \lesssim \min \left(1, s^{-\alpha s}\right)\left\|f_{1}\right\|_{2}\left\|f_{2}\right\|_{2}\left(1+\left\|f_{1}\right\|_{2}^{\gamma_{2}-2}+\left\|f_{2}\right\|_{2}^{\gamma_{2}-2}\right)
$$

for all $0<\alpha<\frac{1}{2}$.

Proof. Because of Lemma 2.7 we have

$$
\begin{aligned}
\mid N\left(f_{1}+f_{2}\right) & -N\left(f_{1}\right)-N\left(f_{2}\right) \mid \\
& \leq \int_{\mathbb{R}} \sum_{x \in \mathbb{Z}}\left|V\left(\left|T_{r} f_{1}(x)+T_{r} f_{2}(x)\right|\right)-V\left(\left|T_{r} f_{1}(x)\right|\right)-V\left(\left|T_{r} f_{2}(x)\right|\right)\right| \mu(d r) \\
& \lesssim M_{\mu}^{\gamma_{1}}\left(f_{1}, f_{2}\right)+M_{\mu}^{\gamma_{2}}\left(f_{1}, f_{2}\right) .
\end{aligned}
$$

So (2.7) follows from (2.3), noting also that

$$
(a+b)^{\gamma_{1}-2}+(a+b)^{\gamma_{2}-2} \lesssim 1+a^{\gamma_{2}-2}+b^{\gamma_{2}-2},
$$

for all $a, b \geq 0$. 


\section{THE EXISTENCE PROOF}

In this section we will give the proof of

Theorem 3.1. Let $\lambda>0$ and assume that $V$ obeys $A 1$ and A2. Then every minimizing sequence for the constrained variational problem (1.5) is precompact modulo translations if and only if $E_{\lambda}^{d_{\mathrm{av}}}<0$. In particular, if $E_{\lambda}^{d_{\mathrm{av}}}<0$, then minimizers of (1.5) exist and these miniminzers are solutions of the diffraction management equation (1.1) for some Lagrange multiplier $\omega<2 E_{\lambda}^{d_{\text {av }}} / \lambda<0$.

Key for our proof of Theorem 3.1 is the following proposition, which will help to eliminate a possible splitting of minimizing sequences when $E_{\lambda}^{d_{\mathrm{av}}}$ is strictly negative. In the following we will assume that the nonlinear potential fulfills assumptions $\mathbf{A} 1$ and $\mathbf{A 2}$. For $s \in \mathbb{R}$, we let $s_{+}:=\max (s, 0)$.

Proposition 3.2. Assume that $V$ obeys $A 1$ and A2. Then there exists a universal constant $C>0$ such that for any $\lambda>0, f \in l^{2}(\mathbb{Z})$ with $\|f\|_{2}^{2}=\lambda$ and $0<\delta<\frac{\lambda}{2}$, and $a, b \in \mathbb{Z}$ with

$$
\sum_{x \leq a}|f(x)|^{2} \geq \delta \text { and } \sum_{x \geq b}|f(x)|^{2} \geq \delta
$$

we have

$$
H(f) \geq\left[1-\left(2^{\frac{\gamma_{0}}{2}}-2\right)\left(\frac{\delta}{\lambda}\right)^{\frac{\gamma_{0}}{2}}\right] E_{\lambda}^{d_{\mathrm{av}}}-C\left(\lambda+\lambda^{\gamma_{2} / 2}\right)\left((b-a+1)_{+}^{1 / 2}-1\right)_{+}^{-1 / 2} .
$$

Remark 3.3. Note that $E_{\lambda}^{d_{\mathrm{av}}} \leq 0$ for all $\lambda>0$ (see Proposition B.2 in the appendix). As soon as $E_{\lambda}^{d_{\mathrm{av}}}<0$ we have

$$
\left[1-\left(2^{\frac{\gamma_{0}}{2}}-2\right)\left(\frac{\delta}{\lambda}\right)^{\frac{\gamma_{0}}{2}}\right] E_{\lambda}^{d_{\mathrm{av}}}>E_{\lambda}^{d_{\mathrm{av}}} .
$$

Therefore if $E_{\lambda}^{d_{\text {av }}}<0$, taking a mininimizing sequence $f_{n}$ with $\left\|f_{n}\right\|_{2}^{2}=\lambda>0$ and $H\left(f_{n}\right) \rightarrow E_{\lambda}^{d_{\mathrm{av}}}$, and taking any $a_{n}$ and $b_{n}$ according to (3.1), the bound (3.2) shows

$$
\limsup _{n \rightarrow \infty}\left(b_{n}-a_{n}\right)<\infty
$$

since $\lim _{s \rightarrow \infty}\left((s+1)_{+}^{1 / 2}-1\right)_{+}^{-1 / 2}=0$. Thus Proposition 3.2 implies that the regions where a minimizing sequence $f_{n}$ has $\delta$-fat tails do not separate too much as soon as the energy $E_{\lambda}^{d_{\mathrm{av}}}$ is strictly negative. This is the key to our proof of compactness modulo translations for minimizing sequences.

Proof. First, let us consider $d_{\mathrm{av}}>0$, that is, strictly positive average diffraction. If $b \leq a$, (3.2) trivially holds since the right hand side of (3.2) equals minus infinity. So assume $b-a \geq 1$. Let $a^{\prime}$ and $b^{\prime}$ be arbitrary integers satisfying $a \leq a^{\prime}<b^{\prime} \leq b$ and $l:=b^{\prime}-a^{\prime}$. We will choose suitable $a^{\prime}$ and $b^{\prime}$ at the end of the proof.

The lower bound on $\langle f,-\Delta f\rangle$ is based on a discrete version of the well-known IMS localization formula, see Lemma C.1 in the appendix. Take any smooth cutoff functions $\tilde{\chi}_{j}, j=-1,0,1$, with

1) $\tilde{\chi}_{j} \geq 0$ for $j=-1,0,1$.

2) $\operatorname{supp} \tilde{\chi}_{-1} \subset\left(-\infty,-\frac{1}{4}\right]$ with $\tilde{\chi}_{-1}>0$ on $\left(-\infty,-\frac{3}{8}\right]$, supp $\tilde{\chi}_{1} \subset\left[\frac{1}{4}, \infty\right)$ with $\tilde{\chi}_{1}>0$ on $\left[\frac{3}{8}, \infty\right)$, and $\operatorname{supp} \tilde{\chi}_{0} \subset\left[-\frac{1}{2}, \frac{1}{2}\right]$ with $\tilde{\chi}_{0}>0$ on $\left[-\frac{3}{8}, \frac{3}{8}\right]$. 
and set

$$
\chi_{j}:=\frac{\tilde{\chi}_{j}}{\sqrt{\sum_{l=-1}^{1} \tilde{\chi}_{l}^{2}}} \text { for } j=-1,0,1 .
$$

Then, since the denominator is always strictly positive and, by construction, $\sum_{j} \chi_{j}^{2}=1$, this gives a smooth partition of unity where $\chi_{j}$ has the same support as $\widetilde{\chi_{j}}$, and $\chi_{0}=1$ on $\left[-\frac{1}{4}, \frac{1}{4}\right], \chi_{-1}=1$ on $\left(-\infty,-\frac{1}{2}\right]$, and $\chi_{1}=1$ on $\left[\frac{1}{2}, \infty\right)$. Finally, define $\xi_{j}: \mathbb{Z} \rightarrow \mathbb{R}$ by

$$
\xi_{j}(x)=\chi_{j}\left(\frac{x-\frac{1}{2}\left(a^{\prime}+b^{\prime}\right)}{b^{\prime}-a^{\prime}}\right) \text { for } j=-1,0,1 .
$$

Then $\sum_{j=-1}^{1} \xi_{j}^{2}=1$ and $\xi_{-1}=1$ on $\left[-\infty, a^{\prime}\right], \xi_{1}=1$ on $\left[b^{\prime}, \infty\right)$ and the supports of $\xi_{-1}$ and $\xi_{1}$ have distance at least $l / 2$, where $l=b^{\prime}-a^{\prime}$. Furthermore, the forward and backward differences $D_{ \pm} f(x)= \pm(f(x \pm 1)-f(x))$ satisfy

$$
\begin{aligned}
\left|D_{ \pm} \xi_{j}(x)\right| & =\left|\xi_{j}(x \pm 1)-\xi_{j}(x)\right|=\left|\chi_{j}\left(\frac{x-\frac{1}{2}\left(a^{\prime}+b^{\prime}\right)}{b^{\prime}-a^{\prime}} \pm \frac{1}{b^{\prime}-a^{\prime}}\right)-\chi_{j}\left(\frac{x-\frac{1}{2}\left(a^{\prime}+b^{\prime}\right)}{b^{\prime}-a^{\prime}}\right)\right| \\
& =\frac{1}{b^{\prime}-a^{\prime}}\left|\chi_{j}^{\prime}(\zeta)\right|
\end{aligned}
$$

for some $\zeta \in \mathbb{R}$. Therefore, since $\chi_{j}^{\prime}$ is bounded, we see that

$$
\left\|D_{ \pm} \xi_{j}\right\|_{\infty}^{2} \leq \frac{C}{3\left(b^{\prime}-a^{\prime}\right)^{2}}=\frac{C}{3 l^{2}}
$$

where $C=3 \max _{j}\left\|\chi_{j}^{\prime}\right\|_{L^{\infty}(\mathbb{R})}^{2}$. Using (3.3) in (C.3), we get

$$
\begin{aligned}
\left\|D_{+} f\right\|_{2}^{2} & =\langle f,-\Delta f\rangle \geq \sum_{j=-1}^{1}\left\|D_{+}\left(\xi_{j} f\right)\right\|_{2}^{2}-\frac{\|f\|_{2}^{2}}{2} \sum_{j=-1}^{1}\left(\left\|D_{+} \xi_{j}\right\|_{\infty}^{2}+\left\|D_{-} \xi_{j}\right\|_{\infty}^{2}\right) \\
& \geq\left\|D_{+}\left(\xi_{-1} f\right)\right\|_{2}^{2}+\left\|D_{+}\left(\xi_{1} f\right)\right\|_{2}^{2}-\frac{C\|f\|_{2}^{2}}{l^{2}} .
\end{aligned}
$$

We set $f_{j}:=\xi_{j} f, j=-1,1$ and define $f_{0}:=f-f_{-1}-f_{1}=\left(1-\xi_{-1}-\xi_{1}\right) f$. Obviously, $\left\|f_{j}\right\|_{2} \leq\|f\|_{2}$ for $j=-1,0,1$. Moreover, because of (3.1) and $a^{\prime} \geq a, b^{\prime} \leq b$, we also have

$$
\left\|f_{j}\right\|_{2}^{2} \geq \delta \quad \text { for } j=-1,1 \text {. }
$$

Set $h:=f_{-1}+f_{1}$. Then $f=h+f_{0}$ and Proposition 2.8 shows

$$
N(f)-N(h)-N\left(f_{0}\right) \lesssim\left\|f_{0}\right\|_{2}\|h\|_{2}\left(1+\left\|f_{0}\right\|_{2}^{\gamma_{2}-2}+\|h\|_{2}^{\gamma_{2}-2}\right) .
$$

Using Proposition 2.6, we have

$$
N\left(f_{0}\right) \lesssim\left\|f_{0}\right\|_{2}^{\gamma_{1}}+\left\|f_{0}\right\|_{2}^{\gamma_{2}} \lesssim\left\|f_{0}\right\|_{2}^{2}\left(1+\left\|f_{0}\right\|_{2}^{\gamma_{2}-2}\right)
$$

and combining the above two bounds we arrive at

$$
N(f)-N(h) \lesssim\left\|f_{0}\right\|_{2}\|f\|_{2}\left(1+\|f\|_{2}^{\gamma_{2}-2}\right)
$$

where we used $\left\|f_{0}\right\|_{2},\|h\|_{2} \leq\|f\|_{2}$.

Since the supports of $f_{-1}$ and $f_{1}$ have distance at least $l / 2=\left(b^{\prime}-a^{\prime}\right) / 2$, we can again use Proposition 2.8 with $\alpha=\frac{1}{4}$ to split $N(h)$ as

$$
\begin{aligned}
N(h)-N\left(f_{-1}\right)-N\left(f_{1}\right) & \lesssim(l / 2)^{-l / 8}\left\|f_{-1}\right\|_{2}\left\|f_{1}\right\|_{2}\left(1+\left\|f_{-1}\right\|_{2}^{\gamma_{2}-2}+\left\|f_{1}\right\|_{2}^{\gamma_{2}-2}\right) \\
& \lesssim(l / 2)^{-l / 8}\|f\|_{2}^{2}\left(1+\|f\|_{2}^{\gamma_{2}-2}\right) .
\end{aligned}
$$


Combining (3.5) and (3.6), we get

$$
N(f)-N\left(f_{-1}\right)-N\left(f_{1}\right) \lesssim\left(\left\|f_{0}\right\|_{2}\|f\|_{2}+(l / 2)^{-l / 8}\|f\|_{2}^{2}\right)\left(1+\|f\|_{2}^{\gamma_{2}-2}\right),
$$

which together with (3.4) yields

$$
H(f)-H\left(f_{-1}\right)-H\left(f_{1}\right) \gtrsim-\left[\frac{\|f\|_{2}^{2}}{l^{2}}+\left(\left\|f_{0}\right\|_{2}\|f\|_{2}+(l / 2)^{-l / 8}\|f\|_{2}^{2}\right)\left(1+\|f\|_{2}^{\gamma_{2}-2}\right)\right] .
$$

Once we have such a bound on the splitting of the energy, we use a reasoning similar to the one in [15]: By definition of $f_{0}$, we have $\left\|f_{0}\right\|_{2}^{2} \leq \sum_{x=a^{\prime}+1}^{b^{\prime}-1}|f(x)|^{2}$. To choose $a^{\prime}$ and $b^{\prime}$, set $I_{\eta}:=\{\eta+1, \eta+2, \ldots, \eta+l-1\}$ when $l \geq 2, I_{\eta}:=\emptyset$ when $l=1$, and note that, since the number of integers in $[a, b-l]$ is $b-a-l+1$,

$$
\begin{aligned}
(b-a-l+1) \min _{a \leq \eta \leq b-l} \sum_{x \in I_{\eta}}|f(x)|^{2} & \leq \sum_{\eta=a}^{b-l} \sum_{x \in I_{\eta}}|f(x)|^{2} \leq \sum_{x=a+1}^{b-1} \sum_{\eta=x-l+1}^{x-1}|f(x)|^{2} \\
& \leq(l-1)\|f\|_{2}^{2} .
\end{aligned}
$$

Hence there exists $\eta^{\prime}$ with $a \leq \eta^{\prime} \leq b-l$ and

$$
\sum_{x=\eta^{\prime}+1}^{\eta^{\prime}+l-1}|f(x)|^{2} \leq \frac{l-1}{b-a-l+1}\|f\|_{2}^{2} .
$$

With $a^{\prime}=\eta^{\prime}$ and $b^{\prime}=\eta^{\prime}+l$ we therefore have

$$
\left\|f_{0}\right\|_{2}^{2} \leq \frac{l-1}{b-a-l+1}\|f\|_{2}^{2}
$$

Plugging this into (3.7) yields

$$
\begin{aligned}
& H(f)-H\left(f_{-1}\right)-H\left(f_{1}\right) \\
& \gtrsim-\left[\frac{\|f\|_{2}^{2}}{l^{2}}+\left(\left(\frac{l-1}{b-a-l+1}\right)^{1 / 2}\|f\|_{2}^{2}+(l / 2)^{-l / 8}\|f\|_{2}^{2}\right)\left(1+\|f\|_{2}^{\gamma_{2}-2}\right)\right] \\
& \geq-\|f\|_{2}^{2}\left(1+\|f\|_{2}^{\gamma_{2}-2}\right)\left[\frac{1}{l^{2}}+\left(\frac{l-1}{b-a-l+1}\right)^{1 / 2}+(l / 2)^{-l / 8}\right] .
\end{aligned}
$$

Since $\|f\|_{2}^{2}=\lambda,\left\|f_{j}\right\|_{2}^{2} \geq \delta, j=-1,1$ and $\left\|f_{-1}\right\|_{2}^{2}+\left\|f_{1}\right\|_{2}^{2} \leq \lambda$, Proposition B.2 shows

$$
H\left(f_{-1}\right)+H\left(f_{1}\right) \geq\left[1-\left(2^{\frac{\gamma_{0}}{2}}-2\right)\left(\frac{\delta}{\lambda}\right)^{\frac{\gamma_{0}}{2}}\right] E_{\lambda}^{d_{\mathrm{av}}}
$$

and inequality (3.8) yields

$$
\begin{aligned}
H(f)- & {\left[1-\left(2^{\frac{\gamma_{0}}{2}}-2\right)\left(\frac{\delta}{\lambda}\right)^{\frac{\gamma_{0}}{2}}\right] E_{\lambda}^{d_{\mathrm{av}}} \gtrsim } \\
& -\left(\lambda+\lambda^{\gamma_{2} / 2}\right)\left[\frac{1}{l^{2}}+\left(\frac{l-1}{b-a-l+1}\right)^{1 / 2}+(l / 2)^{-l / 8}\right]
\end{aligned}
$$

for any $1 \leq l \leq b-a$. 
Finally, we choose $l \in \mathbb{N}$ with $l \leq(b-a+1)^{1 / 2}<l+1$. Note that this is allowed, since when $b-a=1$, we have $l=1$, and when $b-a \geq 2$, then $1 \leq l \leq(b-a+1)^{1 / 2} \leq b-a$. With this choice of $l$ we have

$$
\begin{gathered}
\frac{1}{l^{2}} \leq \frac{1}{l^{1 / 2}} \leq\left((b-a+1)^{1 / 2}-1\right)^{-1 / 2} \\
\left(\frac{l-1}{b-a-l+1}\right)^{1 / 2} \leq\left(\frac{(b-a+1)^{1 / 2}-1}{b-a-(b-a+1)^{1 / 2}+1}\right)^{1 / 2} \leq\left((b-a+1)^{1 / 2}-1\right)^{-1 / 2}
\end{gathered}
$$

and

$$
(l / 2)^{-l / 8} \lesssim l^{-1 / 2} \leq\left((b-a+1)^{1 / 2}-1\right)^{-1 / 2} .
$$

Therefore, (3.9) yields (3.2).

If $d_{\mathrm{av}}=0$, we do not have the term $\frac{1}{l^{2}}$ in (3.9) and get the same estimate (3.2).

An immediate consequence of Proposition 3.2 is

Proposition 3.4 (Tightness). Assume that $E_{\lambda}^{d_{\mathrm{av}}}<0$. Let $\left(f_{n}\right)_{n \in \mathbb{N}} \subset l^{2}(\mathbb{Z})$ be a minimizing sequence for the variational problem (1.1) with $\lambda=\left\|f_{n}\right\|_{2}^{2}>0$. Then there exist shifts $\xi_{n}$ such that

$$
\lim _{R \rightarrow \infty} \sup _{n \in \mathbb{N}} \sum_{\left|x-\xi_{n}\right|>R}\left|f_{n}(x)\right|^{2}=0
$$

Proof. Since the function $s \mapsto(\sqrt{s+1}-1)^{-1 / 2}$ is decreasing on $(0, \infty)$ and goes to zero at infinity, Proposition 3.2 has the same consequences as [15, Proposition 2.4] replacing [15, inequality (2.29)] by (3.2).

To prove Theorem 3.1, we need two more results on the continuity and differentiablity of the non-linear functional $N(f)$. The proof mimics the one in [7] for the continuous case and is therefore omitted.

Lemma 3.5. The functional $N: l^{2}(\mathbb{Z}) \rightarrow \mathbb{R}$ given by

$$
f \mapsto N(f)=\int_{\mathbb{R}} \sum_{x \in \mathbb{Z}} V\left(\left|T_{r} f(x)\right|\right) \mu(d r)
$$

is locally Lipshitz continuous on $l^{2}(\mathbb{Z})$.

Lemma 3.6. For any $f \in l^{2}(\mathbb{Z})$, the functional $N$ as above is continuously differentiable with derivative

$$
l^{2}(\mathbb{Z}) \ni h \mapsto D N(f)[h]=\operatorname{Re} \int_{\mathbb{R}}\left\langle\left[V^{\prime}\left(\left|T_{r} f\right|\right) \operatorname{sgn}\left(T_{r} f\right)\right], T_{r} h\right\rangle \mu(d r),
$$

where $\operatorname{sgn}(z):=\frac{z}{|z|}$ if $z \neq 0$ and $\operatorname{sgn}(0):=0$. In particular, the nonlinear Hamiltonian given in (1.6) is continuously differentiable with derivative

$$
l^{2}(\mathbb{Z}) \ni h \mapsto D H(f)[h]=d_{\mathrm{av}} \operatorname{Re}\left\langle D_{+} f, D_{+} h\right\rangle-\operatorname{Re} \int_{\mathbb{R}}\left\langle\left[V^{\prime}\left(\left|T_{r} f\right|\right) \operatorname{sgn}\left(T_{r} f\right)\right], T_{r} h\right\rangle \mu(d r),
$$

Remark 3.7. Recall that we assume that the nonlinearity $P$ is odd, so it is of the form $P(a)=p(|a|) a$ for $a \in \mathbb{R}$. If $V^{\prime}(a)=P(a)$ for all $a \geq 0$, then $V^{\prime}(|z|) \operatorname{sgn}(z)=p(|z|) z=P(z)$ for all $z \in \mathbb{C}$, and therefore

$$
D N(f)[h]=\operatorname{Re} \int_{\mathbb{R}}\left\langle P\left(T_{r} f\right), T_{r} h\right\rangle \mu(d r)
$$




$$
=\operatorname{Re}\left\langle\int_{\mathbb{R}} T_{r}^{-1}\left[P\left(T_{r} f\right)\right] \mu(d r), h\right\rangle
$$

is, modulo the real part, the weak form of the nonlinearity in the diffraction management equation (1.2).

It remains to prove Theorem 3.1. A last step in our existence proof of minimizers of the variational problems $(1.5)$ is the following characterization of strong convergence in $l^{2}(\mathbb{Z})$.

Lemma 3.8 (Lemma A.1 in [15]). A sequence $\left(f_{n}\right)_{n \in \mathbb{N}} \subset l^{2}(\mathbb{Z})$ is strongly converging to $f$ in $l^{2}(\mathbb{Z})$ if and only if it is weakly convergent to $f$ and the sequence is tight, i.e.,

$$
\lim _{L \rightarrow \infty} \limsup _{n \rightarrow \infty} \sum_{|x|>L}\left|f_{n}(x)\right|^{2}=0
$$

Sketch of the proof: Let $P_{l} f:=\mathbf{1}_{[-l, l]} f$ and note that the range of $P_{l}$ is finite dimensional, in fact, $2 l+1$ dimensional. Thus, if $f_{n}$ converges weakly to $f$, then $\lim _{n \rightarrow \infty}\left\|P_{l}\left(f-f_{n}\right)\right\|_{2}=0$. Since

$$
\left\|f-f_{n}\right\|_{2} \leq\left\|P_{l}\left(f-f_{n}\right)\right\|_{2}+\left\|\left(1-P_{l}\right)\left(f-f_{n}\right)\right\|_{2}
$$

we see that for all $l \in \mathbb{N}$

$$
\limsup _{n \rightarrow \infty}\left\|f-f_{n}\right\|_{2} \leq \limsup _{n \rightarrow \infty}\left\|\left(1-P_{l}\right)\left(f-f_{n}\right)\right\|_{2} \leq\left\|\left(1-P_{l}\right) f\right\|_{2}+\limsup _{n \rightarrow \infty}\left\|\left(1-P_{l}\right) f_{n}\right\|_{2} .
$$

As $l \rightarrow \infty$, the first term goes to zero since $f \in l^{2}(\mathbb{Z})$ and the second goes to zero because of (3.10). So $f_{n}$ converges to $f$ in norm.

Conversely, if $f_{n}$ converges to $f$ in norm, then it is easy to see that it converges to $f$ weakly and (3.10) holds.

Now we can come to the

Proof of Theorem 3.1. We know from Lemma B.1 and B.5 that $-\infty<E_{\lambda}^{d_{\mathrm{av}}} \leq 0$. Assume that $E_{\lambda}^{d_{\mathrm{av}}}=0$ for some $\lambda>0$. Define the sequence $\left(f_{n}\right)_{n}$ by

$$
f_{n}(x):=c_{n} \mathbf{1}_{[-n, n]}(x)
$$

with $c_{n}=\left(\frac{\lambda}{2 n+1}\right)^{1 / 2}$. Then $\left\|f_{n}\right\|_{2}^{2}=\lambda$. Note that $f_{n}$ converges weakly to zero and that any shift of $f_{n}$ also converges weakly to zero. So the sequence $\left(f_{n}\right)_{n}$ is not precompact in $l^{2}(\mathbb{Z})$ modulo translations. Moreover, we have

$$
\left\|D_{+} f_{n}\right\|_{2}^{2}=2 c_{n}^{2} \rightarrow 0 \quad \text { as } n \rightarrow \infty
$$

and, because of (1.9),

$$
\left|N\left(f_{n}\right)\right| \lesssim \int\left(\left\|T_{r} f_{n}\right\|_{\gamma_{1}}^{\gamma_{1}}+\left\|T_{r} f_{n}\right\|_{\gamma_{2}}^{\gamma_{2}}\right) \mu(d r) \lesssim\left\|f_{n}\right\|_{\gamma_{1}}^{\gamma_{1}}+\left\|f_{n}\right\|_{\gamma_{2}}^{\gamma_{2}}
$$

where we also used the bound (A.2) from Lemma A.1. Since for any $\gamma>2$

$$
\left\|f_{n}\right\|_{\gamma}^{\gamma}=\left(\frac{\lambda}{2 n+1}\right)^{\gamma / 2}(2 n+1) \rightarrow 0
$$

as $n \rightarrow \infty$, we have $N\left(f_{n}\right) \rightarrow 0$ as $n \rightarrow \infty$. Thus $f_{n}$ is a minimizing sequence for (1.5) which is not precompact modulo translations. By contrapositive, this shows that if every minimizing sequence is precompact modulo translations, then $E_{\lambda}^{d_{\mathrm{av}}}<0$. 
Conversely, assume that $E_{\lambda}^{d_{\mathrm{av}}}<0$ and let $\left(f_{n}\right)_{n \in \mathbb{N}} \subset l^{2}(\mathbb{Z})$ be a minimizing sequence of the variational problem (1.1). First, applying Proposition 3.4, we see that there exist shifts $\left\{\xi_{n}\right\}$ such that for any $\epsilon>0$ there exists an $R_{\epsilon}>0$ for which

$$
\sum_{\left|x-\xi_{n}\right|>R_{\epsilon}}\left|f_{n}(x)\right|^{2} \leq \epsilon \text { for any } n \in \mathbb{N} .
$$

Define the shifted sequence $\left(\tilde{f}_{n}\right)_{n}$ by $\tilde{f}_{n}(x):=f_{n}\left(x-\xi_{n}\right)$ for $x \in \mathbb{Z}$. It is also a minimizing sequence, due to the invariance of the Hamiltonian $H$ given in (1.6) under shifts.

Noting that $\left(\left\|\tilde{f}_{n}\right\|_{2}\right)$ is bounded as it is a minimizing sequence, we can see there exists a subsequence, also denoted by $\left(\tilde{f}_{n}\right)_{n \in \mathbb{N}}$, which converges weakly to some $\varphi$ in $l^{2}(\mathbb{Z})$. Due to (3.11) the shifted sequence $\left(\tilde{f}_{n}\right)_{n \in \mathbb{N}}$ is tight in the sense of Lemma 3.8, hence by Lemma 3.8 it converges strongly in $l^{2}(\mathbb{Z})$ and $\|\varphi\|_{2}^{2}=\lim _{n \rightarrow \infty}\left\|\tilde{f}_{n}\right\|_{2}^{2}=\lambda$. Thus the minimizing sequence $\left(f_{n}\right)_{n}$ is precompact modulo tranlations.

Moreover, it follows from Lemma 3.5 that $H(\varphi)=\lim _{n \rightarrow \infty} H\left(g_{n}\right)=E_{\lambda}$ which finishes the proof of existence of a minimizer for the constraint variational problem (1.5).

Now we prove that any minimizer is a solution of the associated Euler-Lagrange equation (1.2) for some Lagrange multiplier $\omega \in \mathbb{R}$. This is standard in the calculus of variations, for the convenience of the reader, we will give the argument. Let $\varphi$ be a minimizer for (1.5) and $h \in l^{2}(\mathbb{Z})$ arbitrary. Furthermore define

$$
\begin{aligned}
& G(t, s):=\langle\varphi+t h+s \varphi, \varphi+t h+s \varphi\rangle \\
& F(t, s):=H(\varphi+t h+s \varphi),
\end{aligned}
$$

then a short calculation gives

$$
\nabla G(t, s)=\left(\begin{array}{c}
\partial_{t} G(t, s) \\
\partial_{s} G(t, s)
\end{array}\right)=2\left(\begin{array}{c}
\operatorname{Re}\langle\varphi+t h+s \varphi, \varphi\rangle \\
\operatorname{Re}\langle\varphi+t h+s \varphi, h\rangle
\end{array}\right)
$$

and

$$
\nabla F(t, s)=\left(\begin{array}{c}
\partial_{t} F(t, s) \\
\partial_{s} F(t, s)
\end{array}\right)=\left(\begin{array}{c}
D H(\varphi+t h+s \varphi)[\varphi] \\
D H(\varphi+t h+s \varphi)[h]
\end{array}\right)
$$

where $D H$ is the derivative of the nonlinear Hamiltonian,

$$
\begin{aligned}
D H(\varphi)[h] & =d_{\mathrm{av}} \operatorname{Re}\langle-\Delta \varphi, h\rangle-D N(\varphi)[h] \\
& =d_{\mathrm{av}} \operatorname{Re}\left\langle D_{+} \varphi, D_{+} h\right\rangle-\operatorname{Re} \int_{\mathbb{R}}\left\langle V^{\prime}\left(\left|T_{r} \varphi\right|\right) \operatorname{sgn}\left(T_{r} \varphi\right), T_{r} h\right\rangle \mu(d r) \\
& =d_{\mathrm{av}} \operatorname{Re}\left\langle D_{+} \varphi, D_{+} h\right\rangle-\operatorname{Re} \int_{\mathbb{R}}\left\langle P\left(T_{r} \varphi\right), T_{r} h\right\rangle \mu(d r)
\end{aligned}
$$

where we used Remark 3.7 for the last equality.

We have $\partial_{t} G(0,0)=\langle\varphi, \varphi\rangle=\lambda>0$, hence by the implicit function theorem, there exists $\delta>0$ and a differentiable function $g:(-\delta, \delta) \rightarrow \mathbb{R}$ with $g(0)=0$ such that $G(g(s), s)=$ $G(0,0)=\lambda$ for all $|s|<\delta$. Thus, since $\varphi$ is a minimizer of the constrained minimization problem (1.5), the function

$$
(-\delta, \delta) \ni s \mapsto \tilde{F}(s):=F(g(s), s)
$$

has a local minimum at $s=0$ and together with the chain rule this implies

$$
0=\left.\partial_{s} \tilde{F}(s)\right|_{s=0}=\partial_{t} F(0,0) g^{\prime}(0)+\partial_{s} F(0,0)=D H(\varphi)[\varphi] g^{\prime}(0)+D H(\varphi)[h]
$$

Moreover, since $G(g(s), s)$ is constant, we also have

$$
0=\partial_{t} G(0,0) g^{\prime}(0)+\partial_{s} G(0,0)=2 \lambda g^{\prime}(0)+2 \operatorname{Re}\langle\varphi, h\rangle
$$


solving for $g^{\prime}(0)$ and plugging it back into (3.12) yields

$$
\omega \operatorname{Re}\langle\varphi, h\rangle=d_{\mathrm{av}} \operatorname{Re}\left\langle D_{+} \varphi, D_{+} h\right\rangle-\operatorname{Re} \int_{\mathbb{R}}\left\langle V^{\prime}\left(\left|T_{r} \varphi\right|\right) \operatorname{sgn}\left(T_{r} \varphi\right), T_{r} h\right\rangle \mu(d r)
$$

with the Lagrange multiplier

$$
\omega=\omega(\varphi):=\frac{D H(\varphi)[\varphi]}{\lambda} \in \mathbb{R} .
$$

Replacing $h$ by $-i h$ in (3.13) yields

$$
\omega \operatorname{Im}\langle\varphi, h\rangle=d_{\mathrm{av}} \operatorname{Im}\left\langle D_{+} \varphi, D_{+} h\right\rangle-\operatorname{Im} \int_{\mathbb{R}}\left\langle V^{\prime}\left(\left|T_{r} \varphi\right|\right) \operatorname{sgn}\left(T_{r} \varphi\right), T_{r} h\right\rangle \mu(d r)
$$

and together with (3.13) this proves (1.2).

It remains to prove that $\omega<2 E_{\lambda}^{d_{\mathrm{av}}}$. Recall that assumption A2 states that

$$
V^{\prime}(a) a \geq \gamma_{0} V(a) \text { for all } a>0 \text {. }
$$

Thus

$$
D N(\varphi)[\varphi]=\int_{\mathbb{R}} \sum_{x \in \mathbb{Z}} V^{\prime}\left(\left|T_{r} \varphi(x)\right|\right)\left|T_{r} \varphi(x)\right| \mu(d r) \geq \gamma_{0} \int_{\mathbb{R}} \sum_{x \in \mathbb{Z}} V\left(\left|T_{r} \varphi(x)\right|\right) \mu(d r)=\gamma_{0} N(\varphi)
$$

and since $E_{\lambda}^{d_{\mathrm{av}}}<0$, we must have $N(\varphi)>0$ for any minimizer $\varphi$, so (3.14) gives

$$
\begin{aligned}
\omega(\varphi) \lambda=D H(\varphi)[\varphi] & =d_{\mathrm{av}}\left\langle D_{+} \varphi, D_{+} \varphi\right\rangle-D N(\varphi)[\varphi] \leq d_{\mathrm{av}}\left\langle D_{+} \varphi, D_{+} \varphi\right\rangle-\gamma_{0} N(\varphi) \\
& =2 H(\varphi)-\left(\gamma_{0}-2\right) N(\varphi)<2 H(\varphi)=2 E_{\lambda}^{d_{\mathrm{av}}}<0
\end{aligned}
$$

for all $\varphi$ in the ground state set $\mathcal{M}_{\lambda}^{d_{\mathrm{av}}}$.

\section{Threshold PHENOMENA}

As we showed in the previous section, assumptions A1 and A2 guarantee the existence of minimizers for arbitrary $\lambda>0$ and $d_{\mathrm{av}} \geq 0$ as soon as the ground state energy $E_{\lambda}^{d_{\mathrm{av}}}$ is strictly negative. In this section we will prove a threshold phenomenon: There exists $0 \leq \lambda_{\text {cr }} \leq \infty$ such that solutions exist if the power $\lambda=\|f\|_{2}^{2}>\lambda_{\text {cr }}$. Furthermore $\lambda_{\text {cr }}<\infty$ under assumption A3.

For pure power law nonlinearities and the model case $d_{0}=\mathbf{1}_{[0,1)}-\mathbf{1}_{[1,2]}$ for the diffraction profile, this had been partly investigated earlier in [20] for the diffraction management equation and for pure power nonlinearities in [29] for the discrete nonlinear Schrödinger equation. We are not aware of any work which investigates threshold phenomena for general nonlinearities obeying only A1 and A2.

In the following we will always assume, without explicitly mentioning it every time, that $\mu$ is a finite measure on $\mathbb{R}$ with compact support, that is, there exists $0<B<\infty$ such that $\operatorname{supp} \mu \subset[-B, B]$. Our main result in this section is

Theorem 4.1 (Threshold phenomenon). Assume that $V$ obeys $A 1$ and A2. Then

(i) For any average diffraction $d_{\mathrm{av}} \geq 0$ and any $\lambda>0$ we have $E_{\lambda}^{d_{\mathrm{av}}} \leq 0$, the map $\lambda \mapsto E_{\lambda}^{d_{\mathrm{av}}}$ is decreasing on $(0, \infty)$, and there exists a critical threshold $0 \leq \lambda_{\mathrm{cr}}\left(d_{\mathrm{av}}\right) \leq \infty$ such that for $0<\lambda<\lambda_{\mathrm{cr}}\left(d_{\mathrm{av}}\right)$ we have $E_{\lambda}^{d_{\mathrm{av}}}=0$ and for $\lambda>\lambda_{\mathrm{cr}}\left(d_{\mathrm{av}}\right)$ we have $-\infty<E_{\lambda}^{d_{\mathrm{av}}}<0$.

(ii) If $\lambda>\lambda_{\mathrm{cr}}$, then minimizers of (1.5) exist and any minimizing sequence is, up to translations, precompact in $l^{2}(\mathbb{Z})$ and thus has a subsequence which converges, up to translations, to a minimizer.

(iii) If $0<\lambda<\lambda_{\mathrm{cr}}\left(d_{\mathrm{av}}\right)$ and $d_{\mathrm{av}}>0$, then no minimizers of the variational problem (1.5) 
exist.

(iv) $\lambda_{\mathrm{cr}}\left(d_{\mathrm{av}}\right)<\infty$ for all $d_{\mathrm{av}} \geq 0$ if and only if there exists $f \in l^{2}(\mathbb{Z})$ such that $N(f)>0$.

(v) If in assumption $\boldsymbol{A} 1$ we have $\gamma_{1} \geq 6$, then $\lambda_{c r}\left(d_{\mathrm{av}}\right)>0$ for all $d_{\mathrm{av}}>0$.

Remark 4.2. The precise definition of $\lambda_{\mathrm{cr}}\left(d_{\mathrm{av}}\right)$ is given below in Definition 4.8. When $\lambda>\lambda_{\text {cr }}\left(d_{\text {av }}\right)$ we have $E_{\lambda}^{d_{\mathrm{av}}}<0$ and Theorem 3.1 shows that any minimizing sequence is precompact modulo translations, that minimizers exist and that these minimizers yield solutions of (1.1) for some Lagrange multiplier $\omega<2 E_{\lambda}^{d_{\mathrm{av}}} / \lambda<0$.

Since $E_{\lambda}^{d_{\mathrm{av}}}=0$ when $0<\lambda<\lambda_{\mathrm{cr}}$, Theorem 3.1 also shows that there are minimizing sequences which are not precompact modulo translations in this case. Nevertheless, it could be that minimizers still exist. At least when $d_{\mathrm{av}}>0$, Theorem 4.1 shows that this cannot be the case. At the moment, we need $d_{\mathrm{av}}>0$ to conclude nonexistence of minimizers when $0<\lambda<\lambda_{\mathrm{cr}}$.

We give the proof of Theorem 4.1 at the end of this section after some preparations. Recall

$$
H(f)=\frac{d_{\mathrm{av}}}{2}\left\|D_{+} f\right\|_{2}^{2}-N(f)
$$

and

$$
E_{\lambda}^{d_{\mathrm{av}}}=\inf \left\{H(f): f \in l^{2}(\mathbb{Z}),\|f\|_{2}^{2}=\lambda\right\} .
$$

Given $f \in l^{2}(\mathbb{Z})$ with $\lambda=\|f\|_{2}^{2}>0$, write it as $f=\sqrt{\lambda} h$ then $h \in l^{2}(\mathbb{Z})$ with $\|h\|_{2}=1$ and

$$
H(f)=\frac{d_{\mathrm{av}}}{2}\left\|D_{+} f\right\|_{2}^{2}-N(f)=\left\|D_{+} f\right\|_{2}^{2}\left(\frac{d_{\mathrm{av}}}{2}-\frac{N(\sqrt{\lambda} h)}{\lambda\left\|D_{+} h\right\|_{2}^{2}}\right) .
$$

In the case of vanishing average diffraction, we can still write

$$
H(f)=-N(f)=-\left\|D_{+} f\right\|_{2}^{2}\left(\frac{N(\sqrt{\lambda} h)}{\lambda\left\|D_{+} h\right\|_{2}^{2}}\right),
$$

so defining ${ }^{3}$

$$
R(\lambda, h):=\frac{N(\sqrt{\lambda} h)}{\lambda\left\|D_{+} h\right\|_{2}^{2}}
$$

and

$$
R(\lambda):=\sup _{\|h\|_{2}=1} R(\lambda, h)=\sup _{\|f\|_{2}^{2}=\lambda} \frac{N(f)}{\left\|D_{+} f\right\|_{2}^{2}}
$$

we see that the following holds

Lemma 4.3. For any $d_{\mathrm{av}} \geq 0$ and $\lambda>0$ one has $E_{\lambda}^{d_{\mathrm{av}}}<0$ if and only if $R(\lambda, h)>\frac{d_{\mathrm{av}}}{2}$ for some $h \in l^{2}(\mathbb{Z})$ with $\|h\|_{2}=1$ and this is the case if and only if $R(\lambda)>\frac{d_{\mathrm{av}}}{2}$.

The function $R$ defined above has very interesting properties, which make $R$ ideal for the study of the threshold phenomenon. First we give a simple Lemma, which is at the heart of our study of $R$.

Lemma 4.4. Assume that $V$ obeys assumption A2. Then for any $\lambda_{2} \geq \lambda_{1}>0$ one has

$$
R\left(\lambda_{2}\right) \geq\left(\frac{\lambda_{2}}{\lambda_{1}}\right)^{\frac{\gamma_{0}-2}{2}} R\left(\lambda_{1}\right) .
$$

\footnotetext{
${ }^{3}$ Note that the kernel of $D_{+}$on $l^{2}(\mathbb{Z})$ is trivial, so $R(\lambda, h)$ is well defined for any $h \neq 0$.
} 
Remark 4.5. For a pure power law nonlinearity, given by $V(a)=c a^{\gamma}$ for some $\gamma>2$ and $c>0$, one can explicitly calculate

$$
R(\lambda)=\sup _{\|h\|_{2}=1} \frac{N(\sqrt{\lambda} h)}{\lambda\left\|D_{+} h\right\|_{2}^{2}}=\lambda^{\frac{\gamma-2}{2}} R_{0} \quad \text { with } R_{0}=\sup _{\|h\|_{2}=1} \frac{N(h)}{\left\|D_{+} h\right\|_{2}^{2}} \in(0, \infty] .
$$

Thus inequality (4.3) is very natural. Using the bound (4.17) below one sees that

$$
R_{0} \leq \sup _{\|h\|_{2}=1} c \int_{\mathbb{R}}\left\|T_{r} h\right\|_{2}^{\gamma-2} \mu(d r)=c \mu(\mathbb{R})<\infty
$$

for all $\gamma \geq 6$ since $\left\|D_{+} T_{r} h\right\|_{2}^{2}=\left\langle T_{r} h,-\Delta T_{r} h\right\rangle=\langle h,-\Delta h\rangle=\left\|D_{+} h\right\|_{2}^{2}$, using that $\Delta$ and $T_{r}$ commute. To see that $R_{0}=\infty$ if $2<\gamma<6$ is a little bit trickier. If $2<\gamma<6$, then Lemma B.5 shows $E_{\lambda}^{d_{\mathrm{av}}}<0$ for all $d_{\mathrm{av}} \geq 0$ and all $\lambda>0$. So with Lemma 4.3 for $\lambda=1$ this gives $R_{0}=R(1)>d_{\mathrm{av}} / 2$ for all $d_{\mathrm{av}} \geq 0$. Thus $R_{0}=\infty$ in this case.

Proof of Lemma 4.4. Fix $h \in l^{2}(\mathbb{Z}) \backslash\{0\}$ and define

$$
A(s):=s^{-2} N(s h)
$$

for $s>0$. Because of Lemma 3.6, $A$ is differentiable with derivative

$$
A^{\prime}(s)=s^{-3}(D N(s h)[s h]-2 N(s h))
$$

where

$$
\begin{aligned}
D N(s h)[s h]-2 N(s h) & =\int_{\mathbb{R}} \sum_{x \in \mathbb{Z}}\left[V^{\prime}\left(\left|T_{r}(s h)(x)\right|\right)\left|T_{r}(s h)(x)\right|-2 V\left(\left|T_{r}(s h)(x)\right|\right)\right] \mu(d r) \\
& \geq\left(\gamma_{0}-2\right) N(s h)
\end{aligned}
$$

where the lower bound follows from assumption A2. Thus we arrive at the first order differential inequality

$$
A^{\prime}(s) \geq \frac{\gamma_{0}-2}{s} A(s)
$$

for all $s>0$. Using the integrating factor $s^{2-\gamma_{0}}$, one sees that this implies $\frac{d}{d s}\left(s^{2-\gamma_{0}} A(s)\right) \geq 0$ and thus

$$
s^{2-\gamma_{0}} A(s) \geq s_{0}^{2-\gamma_{0}} A\left(s_{0}\right)
$$

for all $0<s_{0} \leq s$. Since $R(\lambda, h)=A(\sqrt{\lambda}) /\left\|D_{+} h\right\|_{2}^{2}$, this proves

$$
R\left(\lambda_{2}, h\right) \geq\left(\frac{\lambda_{2}}{\lambda_{1}}\right)^{\frac{\gamma_{0}-2}{2}} R\left(\lambda_{1}, h\right) .
$$

for all $0<\lambda_{1} \leq \lambda_{2}$ and taking the supremum over all $h \in l^{2}(\mathbb{Z})$ with $\|h\|_{2}=1$ gives (4.3).

Corollary 4.6 (Properties of R). Assume that $V$ obeys Assumption A2.

(i) For any $0 \leq a \leq \infty$

there exist $\lambda_{0}>0$ with $R\left(\lambda_{0}\right) \geq a \Rightarrow R(\lambda) \geq a$ for all $\lambda \geq \lambda_{0}$

there exist $\lambda_{0}>0$ with $R\left(\lambda_{0}\right) \leq a \Rightarrow R(\lambda) \leq a$ for all $0<\lambda \leq \lambda_{0}$

Moreover, for any $0<a<\infty$

there exist $\lambda_{0}>0$ with $R\left(\lambda_{0}\right) \geq a \Rightarrow R(\lambda)>a$ for all $\lambda>\lambda_{0}$

there exist $\lambda_{0}>0$ with $R\left(\lambda_{0}\right) \leq a \Rightarrow R(\lambda)<a$ for all $0<\lambda<\lambda_{0}$. 
Furthermore, we have the equivalences

$$
\begin{array}{lll}
\text { there exists } \lambda>0 \text { with } R(\lambda)>0 & \Leftrightarrow & \lim _{\lambda \rightarrow \infty} R(\lambda)=\infty \\
\text { there exists } \lambda>0 \text { with } R(\lambda)<\infty & \Leftrightarrow & \lim _{\sup _{\lambda \rightarrow 0+}} R(\lambda) \leq 0 .
\end{array}
$$

(ii) Define the set $A_{0}:=\{\lambda>0: R(\lambda)>0\}$, then it is either empty or an unbounded interval. Moreover, the map $R$ is increasing on $A_{0}$ and it is strictly increasing where it is finite.

Remarks 4.7. (i) Even though Lemma B.1 shows that under Assumption A1 the energy is negative, this is not enough to conclude that $R(\lambda) \geq 0$ for all $\lambda>0$, in general.

(ii) All the conclusions of Corollary 4.6 are trivially true if $V(a)=c a^{\gamma}$ is a pure power law for some $\gamma>2$ and $c>0$, since in this case $R(\lambda)=R_{0} \lambda^{(\gamma-2) / 2}$ as in Remark 4.5.

(iii) The first equivalence in (4.7) shows that we have the dichotomy that either $R(\lambda) \leq 0$ for all $\lambda>0$, or $\lim _{\lambda \rightarrow \infty} R(\lambda)=\infty$.

Similarly, the second equivalence in (4.7) shows the dichotomy that either $R(\lambda)=\infty$ for all $\lambda>0$ or $\lim \sup _{\lambda \rightarrow 0+} R(\lambda) \leq 0$.

Proof of Corollary 4.6. (i) The implications of (4.5) and (4.6) follow directly from Lemma 4.4. If $\lambda>\lambda_{0}$, then choosing $\lambda_{1}=\lambda_{0}$ and $\lambda_{2}=\lambda$ in (4.3) shows

$$
R(\lambda) \geq\left(\frac{\lambda}{\lambda_{0}}\right)^{\frac{\gamma_{0}-2}{2}} R\left(\lambda_{0}\right) .
$$

Let $0<a<\infty$. If $R\left(\lambda_{0}\right)=\infty$ we also have $R(\lambda)=\infty$ for all $\lambda \geq \lambda_{0}$. If $R\left(\lambda_{0}\right)=\infty$, then (4.8) shows $R(\lambda)=\infty>a$. If $a \leq R\left(\lambda_{0}\right)<\infty$, then necessarily $R\left(\lambda_{0}\right)>0$, hence $\left(\frac{\lambda}{\lambda_{0}}\right)^{\frac{\gamma_{0}-2}{2}} R\left(\lambda_{0}\right)>R\left(\lambda_{0}\right)$ and (4.8) again gives $R(\lambda)>a$. So the first implication of (4.6) is true. Hence also the first implication of (4.5) is true when $a$ is strictly positive and finite, but when $a=\infty$ or $a=0$, the first implication of (4.5) immediately follows from (4.8). This finishes the proof of the first implications in (4.5) and (4.6).

Now let $0<\lambda<\lambda_{0}$. Choosing $\lambda_{1}=\lambda$ and $\lambda_{2}=\lambda_{0}$ in (4.3) gives the upper bound

$$
R(\lambda) \leq\left(\frac{\lambda}{\lambda_{0}}\right)^{\frac{\gamma_{0}-2}{2}} R\left(\lambda_{0}\right),
$$

If $0<a<\infty$ and $R\left(\lambda_{0}\right)=0$, then (4.9) shows $R(\lambda) \leq 0<a$. If $0<R\left(\lambda_{0}\right) \leq a$, then $\left(\frac{\lambda}{\lambda_{0}}\right)^{\frac{\gamma_{0}-2}{2}} R\left(\lambda_{0}\right)<R\left(\lambda_{0}\right)$, so (4.9) again yields $R(\lambda)<a$. This proves the second implication in (4.6). The second implication of (4.5) when $a=\infty$ or $a=0$ immediately follows from (4.9). This finishes the proof of (4.5) and (4.6).

For the proof of (4.7) assume first that $\lim _{\lambda \rightarrow \infty} R(\lambda)=\infty$. Then, of course, there exists $\lambda>0$ with $R(\lambda)>0$. On the other hand, if there exists $\lambda_{0}>0$ such that $R\left(\lambda_{0}\right)>0$, then the lower bound (4.8) gives $\liminf _{\lambda \rightarrow \infty} R(\lambda)=\infty$, so the first equivalence in (4.7) is true.

We can argue similarly for the second equivalence. Certainly $\lim \sup _{\lambda \rightarrow 0+} R(\lambda) \leq 0$ implies that there exists $\lambda>0$ such that $R(\lambda)<\infty$. Conversely, if $R\left(\lambda_{0}\right)<\infty$ for some $\lambda_{0}$, then (4.9) yields $\lim \sup _{\lambda \rightarrow 0+} R(\lambda) \leq 0$. This finishes (4.7).

(ii) Note that if $\lambda_{0} \in A_{0}$, then (4.6) yields $\lambda \in A_{0}$ for all $\lambda>\lambda_{0}$, so

$$
A_{0}=\bigcup_{R(\lambda)>0}[\lambda, \infty)
$$


is either empty or an unbounded interval. Moreover, the first implication of (4.5) shows that $R$ is increasing on $A_{0}$ and the first implication of (4.6) shows that it is strictly increasing where it is finite.

Now we come to our definition of the threshold.

Definition 4.8 (Threshold). For $d_{\mathrm{av}} \geq 0$ we let

$$
\lambda_{\mathrm{cr}}:=\lambda_{\mathrm{cr}}\left(d_{\mathrm{av}}\right):=\inf \left\{\lambda>0: R(\lambda)>\frac{d_{\mathrm{av}}}{2}\right\} .
$$

For the properties of the threshold, we note

Proposition 4.9 (Properties of the threshold). Assume that $V$ obeys A2.

(i) The map $d_{\mathrm{av}} \mapsto \lambda_{\mathrm{cr}}\left(d_{\mathrm{av}}\right)$ is increasing on $[0, \infty)$ and $0 \leq \lambda_{\mathrm{cr}}\left(d_{\mathrm{av}}\right) \leq \infty$ for every $d_{\mathrm{av}} \geq 0$.

(ii) If $d_{\mathrm{av}} \geq 0$ then $R(\lambda)>\frac{d_{\mathrm{av}}}{2}$ for all $\lambda>\lambda_{\mathrm{cr}}\left(d_{\mathrm{av}}\right)$ and $R(\lambda) \leq \frac{d_{\mathrm{av}}}{2}$ for all $0<\lambda<\lambda_{\mathrm{cr}}\left(d_{\mathrm{av}}\right)$. Furthermore, if $d_{\mathrm{av}}>0$ then $R(\lambda)<\frac{d_{\mathrm{av}}}{2}$ for all $0<\lambda<\lambda_{\mathrm{cr}}\left(d_{\mathrm{av}}\right)$.

(iii) We have the equivalences

$$
\begin{aligned}
\lambda_{\mathrm{cr}}\left(d_{\mathrm{av}}\right)<\infty \text { for all } d_{\mathrm{av}} \geq 0 & \Leftrightarrow \lambda_{\mathrm{cr}}\left(d_{\mathrm{av}}\right)<\infty \text { for some } d_{\mathrm{av}} \geq 0 \\
& \Leftrightarrow R(\lambda)>0 \text { for some } \lambda>0, \\
& \Leftrightarrow \lim _{\lambda \rightarrow \infty} R(\lambda)=\infty
\end{aligned}
$$

and

$$
\begin{aligned}
\lambda_{\mathrm{cr}}\left(d_{\mathrm{av}}\right)>0 \text { for all } d_{\mathrm{av}}>0 & \Leftrightarrow \lambda_{\mathrm{cr}}\left(d_{\mathrm{av}}\right)>0 \text { for some } d_{\mathrm{av}} \geq 0 . \\
& \Leftrightarrow \lim \sup _{\lambda \rightarrow 0+} R(\lambda) \leq 0
\end{aligned}
$$

For zero average diffraction we have

$$
\begin{aligned}
& \lambda_{\mathrm{cr}}(0)=0 \quad \Leftrightarrow \quad R(\lambda)>0 \text { for all } \lambda>0 \\
& \lambda_{\mathrm{cr}}(0)>0 \quad \Leftrightarrow \quad R(\lambda) \leq 0 \text { for some } \lambda>0 .
\end{aligned}
$$

Remark 4.10. A moments reflection shows that $R(\lambda)>0$ for some $\lambda>0$ if and only if there exists $f \in l^{2}(\mathbb{Z})$ with $\|f\|_{2}^{2}=\lambda$ and $N(f)>0$. So by (4.10) one sees that the critical threshold $\lambda_{\text {cr }}\left(d_{\mathrm{av}}\right)$ is finite for all $d_{\mathrm{av}} \geq 0$ if and only if $N(f)>0$ for some $f \in l^{2}(\mathbb{Z})$.

Before we prove the proposition we state and prove a corollary, which gives quantitive bounds on the threshold. We do not need these bounds in the following, but the proof is easy and the bounds are very natural, as the example of a pure power nonlinearity shows.

Corollary 4.11 (Quantitative bounds on $\lambda_{\mathrm{cr}}$ ). Assume that $V$ obeys A2. If there exist $\lambda_{0}$ and $0<R_{0}<\infty$ such that $R_{0} \geq R\left(\lambda_{0}\right)$ then we have the lower bound

$$
\lambda_{0}\left(\min \left(\frac{d_{\mathrm{av}}}{2 R_{0}}, 1\right)\right)^{\frac{2}{\gamma_{0}-2}} \leq \lambda_{\mathrm{cr}}\left(d_{\mathrm{av}}\right) \quad \text { for all } d_{\mathrm{av}}>0
$$

and if there exist $\lambda_{0}$ and $0<R_{0}<\infty$ such that $R_{0} \leq R\left(\lambda_{0}\right)$ then we have the upper bound

$$
\lambda_{\mathrm{cr}}\left(d_{\mathrm{av}}\right) \leq \lambda_{0}\left(\max \left(\frac{d_{\mathrm{av}}}{2 R_{0}}, 1\right)\right)^{\frac{2}{\gamma_{0}-2}} \quad \text { for all } d_{\mathrm{av}}>0 .
$$

Remark 4.12. If $V(a)=c a^{\gamma}$ is a pure power law for some $\gamma>2$ and $c>0$, then by Remark 4.5 we have $R(\lambda)=R_{0} \lambda^{(\gamma-2) / 2}$ for some $0<R_{0} \leq \infty$. In this case one can easily calculates

$$
\lambda_{\mathrm{cr}}\left(d_{\mathrm{av}}\right)=\left(\frac{d_{\mathrm{av}}}{2 R_{0}}\right)^{\frac{2}{\gamma-2}}
$$


and with this example in mind one sees that the bounds of Corollary 4.11 and the claims of Proposition 4.9 are very natural.

Proof. Since the proofs of (4.13) and (4.14) are very analogous, we give only the proof of (4.13). Assume that there exist $\lambda_{0}$ and $0<R_{0}<\infty$ with $R\left(\lambda_{0}\right) \leq R_{0}$. By (4.5) with $a=R_{0}$ we see that $R(\lambda) \leq R_{0}$ for all $0<\lambda \leq \lambda_{0}$, so $\left(\lambda_{0}, \infty\right) \supset\left\{\lambda>0: R(\lambda)>R_{0}\right\}$. This shows $\lambda_{\text {cr }}\left(2 R_{0}\right) \geq \lambda_{0}$ for $d_{\mathrm{av}}=2 R_{0}$ and using the monotonicity in $d_{\mathrm{av}}$ from Proposition (i) we also have $\lambda_{\mathrm{cr}}\left(d_{\mathrm{av}}\right) \geq \lambda_{\mathrm{cr}}\left(2 R_{0}\right) \geq \lambda_{0}$ for all $d_{\mathrm{av}} \geq 2 R_{0}$.

Now let $0<d_{\mathrm{av}}<2 R_{0}$ and write $\lambda_{\mathrm{cr}}$ for $\lambda_{\mathrm{cr}}\left(d_{\mathrm{av}}\right)$. Either we have $\lambda_{\mathrm{cr}} \geq \lambda_{0}$, then (4.13) trivially holds, or $0 \leq \lambda_{c r}<\lambda_{0}$. In the last case set $\lambda_{2}=\lambda_{0}$ and $0<\lambda_{1}=\lambda_{\text {cr }}+\delta<\lambda_{0}$ for all small enough $\delta>0$, then Proposition (ii) shows $R\left(\lambda_{c r}+\delta\right)>\frac{d_{\mathrm{av}}}{2}$ which together with (4.3) gives

$$
R_{0} \geq R\left(\lambda_{0}\right) \geq\left(\frac{\lambda_{0}}{\lambda_{\mathrm{cr}}+\delta}\right)^{\frac{\gamma_{0}-2}{2}} R\left(\lambda_{\mathrm{cr}}+\delta\right)>\left(\frac{\lambda_{0}}{\lambda_{\mathrm{cr}}+\delta}\right)^{\frac{\gamma_{0}-2}{2}} \frac{d_{\mathrm{av}}}{2}
$$

for all small enough $\delta>0$. This proves the lower bound (4.13) and similarly one proves the upper bound (4.14).

Proof of Proposition 4.9. First some preparations. For $d_{\mathrm{av}} \geq 0$, define the set

$$
A_{d_{\mathrm{av}}}:=\left\{\lambda>0: R(\lambda)>\frac{d_{\mathrm{av}}}{2}\right\}
$$

so that $\lambda_{\mathrm{cr}}\left(d_{\mathrm{av}}\right)=\inf A_{d_{\mathrm{av}}}$. Arguing as in the proof of Corollary 4.6(ii) we see that if $\lambda_{0} \in A_{d_{\mathrm{av}}}$, then $\lambda \in A_{d_{\mathrm{av}}}$ for all $\lambda \geq \lambda_{0}$. Hence

$$
A_{d_{\mathrm{av}}}=\bigcup_{R\left(\lambda_{0}\right)>\frac{d_{\mathrm{av}}}{2}}\left[\lambda_{0}, \infty\right)
$$

and so the set $A_{d_{\mathrm{av}}}$ is either empty, or an interval that is bounded from below by 0 but unbounded from above, and they are nested, in the sense that if $0 \leq d_{\mathrm{av}, 1} \leq d_{\mathrm{av}, 2}$ then $A_{d_{\mathrm{av}, 2}} \subset A_{d_{\mathrm{av}, 1}}$. In addition, $A_{d_{\mathrm{av}}}$ is empty if and only if the threshold $\lambda_{\mathrm{cr}}\left(d_{\mathrm{av}}\right)=\infty, A_{d_{\mathrm{av}}}$ is not empty if and only if $0 \leq \lambda_{\mathrm{cr}}\left(d_{\mathrm{av}}\right)<\infty$, and $A_{d_{\mathrm{av}}}=(0, \infty)$ if and only if $\lambda_{\mathrm{cr}}\left(d_{\mathrm{av}}\right)=0$.

(i) Since, by the above $A_{d_{\mathrm{av}, 2}} \subset A_{d_{\mathrm{av}, 1}}$ for $0 \leq d_{\mathrm{av}, 1} \leq d_{\mathrm{av}, 2}$, we immediately see $\lambda_{\mathrm{cr}}\left(d_{\mathrm{av}, 1}\right) \leq \lambda_{\mathrm{cr}}\left(d_{\mathrm{av}, 2}\right)$.

(ii) First let $d_{\mathrm{av}} \geq 0$. Certainly, if $R(\lambda)>\frac{d_{\mathrm{av}}}{2}$ then $\lambda \geq \lambda_{\mathrm{cr}}\left(d_{\mathrm{av}}\right)$, so, by contrapositive, if $0<\lambda<\lambda_{\mathrm{cr}}\left(d_{\mathrm{av}}\right)$ then $R(\lambda) \leq \frac{d_{\mathrm{av}}}{2}$. Moreover, if $\lambda>\lambda_{\mathrm{cr}}\left(d_{\mathrm{av}}\right)$, then (4.15) shows $\lambda \in A_{d_{\mathrm{av}}}$, so $R(\lambda)>\frac{d_{\mathrm{av}}}{2}$. This proves the first claim.

Now let $d_{\mathrm{av}}>0$ and $0<\lambda<\lambda_{\text {cr }}\left(d_{\mathrm{av}}\right)$. Then, by the first claim, we already know $R(\lambda) \leq \frac{d_{\mathrm{av}}}{2}$. Moreover, if we suppose that $R(\lambda)=\frac{d_{\mathrm{av}}}{2}$ then (4.6) yields a contradiction to the fact that $\lambda_{\mathrm{cr}}\left(d_{\mathrm{av}}\right)$ is a lower bound for $A_{d_{\mathrm{av}}}$. Thus $R(\lambda)<\frac{d_{\mathrm{av}}}{2}$ and this proves the second claim.

(iii) We certainly have that $\lambda_{\mathrm{cr}}\left(d_{\mathrm{av}}\right)<\infty$ for all $d_{\mathrm{av}} \geq 0$ implies $\lambda_{\mathrm{cr}}\left(d_{\mathrm{av}}\right)<\infty$ for some $d_{\mathrm{av}} \geq 0$. Next, if there exists $d_{\mathrm{av}} \geq 0$ such that $\lambda_{\mathrm{cr}}\left(d_{\mathrm{av}}\right)<\infty$ then $A_{d_{\mathrm{av}}}$ is not empty, hence $R(\lambda)>\frac{d_{\mathrm{av}}}{2} \geq 0$ for some $\lambda>0$. Thirdly, if there exists $\lambda>0$ such that $R(\lambda)>0$ then, (4.7) gives $\lim _{\lambda \rightarrow \infty} R(\lambda)=\infty$. Lastly, if $\lim _{\lambda \rightarrow \infty} R(\lambda)=\infty$, then, for every $d_{\text {av }} \geq 0$, we have $R(\lambda)>\frac{d_{\mathrm{av}}}{2}$ for all large enough $\lambda$ which shows that $A_{d_{\mathrm{av}}}$ is not empty, hence $\lambda_{\mathrm{cr}}\left(d_{\mathrm{av}}\right)<\infty$ for all $d_{\mathrm{av}} \geq 0$. This finishes the proof of (4.10).

For the proof of (4.11) we note that certainly $\lambda_{\mathrm{cr}}\left(d_{\mathrm{av}}\right)>0$ for all $d_{\mathrm{av}}>0$ implies $\lambda_{\mathrm{cr}}\left(d_{\mathrm{av}}\right)>0$ for some $d_{\mathrm{av}} \geq 0$. Next, if there exists $d_{\mathrm{av}} \geq 0$ such that $\lambda_{\mathrm{cr}}\left(d_{\mathrm{av}}\right)>0$ then $R(\lambda) \leq \frac{d_{\mathrm{av}}}{2}<\infty$ for some $\lambda>0$ and so, by $(4.7), \lim _{\sup _{\lambda \rightarrow 0+}} R(\lambda) \leq 0$. Lastly, if 
$\lim \sup _{\lambda \rightarrow 0+} R(\lambda) \leq 0$ then, for every $d_{\mathrm{av}}>0$, we have $R(\lambda) \leq \frac{d_{\mathrm{av}}}{2}$ for all small enough $\lambda>0$, and hence $\lambda_{\mathrm{cr}}\left(d_{\mathrm{av}}\right)>0$ for every $d_{\mathrm{av}}>0$. This finishes the proof of (4.11).

For the proof of (4.12) recall that $\lambda_{\text {cr }}(0)=0$ if and only if $A_{0}=(0, \infty)$, which is the case if and only if $R(\lambda)>0$ for any $\lambda>0$. Moreover, $\lambda_{\mathrm{cr}}(0)>0$ if and only if $A_{0} \neq(0, \infty)$, that is, if and only if there exists $\lambda>0$ with $R(\lambda) \leq 0$.

Now we can give the

Proof of Theorem 4.1. (i)+(ii) Fix $d_{\mathrm{av}} \geq 0$. It follows from Lemma B.1 and Proposition B.2 that $-\infty<E_{\lambda}^{d_{\mathrm{av}}} \leq 0$, for every $d_{\mathrm{av}} \geq 0$ and $\lambda>0$, and the map $\lambda \mapsto E_{\lambda}^{d_{\mathrm{av}}}$ is decreasing on $(0, \infty)$. Proposition 4.9 gives the existence of a critical threshold $0 \leq \lambda_{\mathrm{cr}}=\lambda_{\mathrm{cr}}\left(d_{\mathrm{av}}\right) \leq \infty$ such that if $\lambda>\lambda_{\mathrm{cr}}$, we have $R(\lambda)>\frac{d_{\mathrm{av}}}{2}$. In this case, Lemma 4.3 shows that $E_{\lambda}^{d_{\mathrm{av}}}<0$. Moreover, if $0<\lambda<\lambda_{\mathrm{cr}}\left(d_{\mathrm{av}}\right)$, then Proposition 4.9 and Lemma 4.3 also show that $E_{\lambda}^{d_{\mathrm{av}}} \geq 0$ and so $E_{\lambda}^{d_{\mathrm{av}}}=0$ for all $0<\lambda<\lambda_{\mathrm{cr}}\left(d_{\mathrm{av}}\right)$. This proves the first part of Theorem 4.1 and Theorem 3.1 yields the claims of its second part.

(iii) Let $d_{\mathrm{av}}>0$ and $0<\lambda<\lambda_{\mathrm{cr}}$. If $f \in l^{2}(\mathbb{Z})$ with $\|f\|_{2}^{2}=\lambda>0$ is a minimizer, then using (4.1) gives

$$
0=E_{\lambda}^{d_{\mathrm{av}}}=H(f) \geq\left\|D_{+} f\right\|^{2}\left(\frac{d_{\mathrm{av}}}{2}-R(\lambda)\right) .
$$

By Proposition 4.9(ii), we have $R(\lambda)<\frac{d_{\mathrm{av}}}{2}$, so the inequality (4.16) implies $\left\|D_{+} f\right\|_{2}^{2} \leq 0$, that is, $\left\|D_{+} f\right\|_{2}^{2}=0$. Since the kernel of $D_{+}$is trivial this shows $f=0$, which contradicts $\|f\|_{2}^{2}=\lambda>0$, so no minimizers can exist in this case.

(iv) By Remark 4.10 we have $\lambda_{\text {cr }}<\infty$ if and only if there exist $f \in l^{2}(\mathbb{Z})$ with $N(f)>0$.

(v) We have to show that if $\gamma_{1} \geq 6$, then $\lambda_{\mathrm{cr}}\left(d_{\mathrm{av}}\right)>0$ for all $d_{\mathrm{av}}>0$. For this we use the inequality

$$
\|f\|_{\gamma}^{\gamma} \leq\|f\|_{2}^{\gamma-2}\left\|D_{+} f\right\|_{2}^{2}
$$

which holds for all $f \in l^{2}(\mathbb{Z})$ and all $\gamma \geq 6$. Assuming (4.17) for the moment, one can argue as follows: From (4.17) we have, under assumptions A1 with $\gamma_{1} \geq 6$,

$$
\begin{aligned}
|N(f)| & \leq \int_{\mathbb{R}}\left\|V\left(\left|T_{r} f\right|\right)\right\|_{1} \mu(d r) \lesssim \int_{\mathbb{R}}\left(\left\|T_{r} f\right\|_{2}^{\gamma_{1}-2}+\left\|T_{r} f\right\|_{2}^{\gamma_{2}-2}\right)\left\|D_{+} T_{r} f\right\|_{2}^{2} \mu(d r) \\
& \lesssim\left(\|f\|_{2}^{\gamma_{1}-2}+\|f\|_{2}^{\gamma_{2}-2}\right)\left\|D_{+} f\right\|_{2}^{2} .
\end{aligned}
$$

So

$$
|R(\lambda)|=\sup _{\|f\|_{2}^{2}=\lambda} \frac{|N(f)|}{\left\|D_{+} f\right\|_{2}^{2}} \lesssim \lambda^{\frac{\gamma_{1}-2}{2}}+\lambda^{\frac{\gamma_{2}-2}{2}}<\infty
$$

which directly shows $\lim _{\lambda \rightarrow 0+} R(\lambda)=0$ and then (4.11) gives $\lambda_{\mathrm{cr}}\left(d_{\mathrm{av}}\right)>0$ for all $d_{\mathrm{av}}>0$.

It remains to prove (4.17). For this we recall

$$
\|f\|_{\infty}^{2} \leq\|f\|_{2}\left\|D_{+} f\right\|_{2}
$$

for any $f \in l^{2}(\mathbb{Z})$. Indeed, to see this let $x \in \mathbb{Z}$, then

$$
|f(x)|^{2}=\sum_{l \leq x}\left(|f(l)|^{2}-|f(l-1)|^{2}\right)=\sum_{l \leq x}(|f(l)|+|f(l-1)|)(|f(l)|-|f(l-1)|)
$$


and similarly,

$$
|f(x)|^{2}=-\sum_{l>x}\left(|f(l)|^{2}-|f(l-1)|^{2}\right)=-\sum_{l>x}(|f(l)|+|f(l-1)|)(|f(l)|-|f(l-1)|) .
$$

Adding this two inequalities and using Cauchy-Schwarz gives

$$
\begin{aligned}
|f(x)|^{2} & \leq \frac{1}{2} \sum_{l \in \mathbb{Z}}(|f(l)|+|f(l-1)|)|| f(l)|-| f(l-1)|| \\
& \leq \frac{1}{2} \sum_{l \in \mathbb{Z}}(|f(l)|+|f(l-1)|)|f(l)-f(l-1)| \\
& \leq\left(\sum_{l \in \mathbb{Z}}|f(l)|^{2}\right)^{1 / 2}\left(\sum_{l \in \mathbb{Z}}|f(l)-f(l-1)|^{2}\right)^{1 / 2}=\|f\|_{2}\left\|D_{+} f\right\|_{2}
\end{aligned}
$$

which, since it holds for all $x \in \mathbb{Z}$, gives (4.18). From (4.18), we see that for $\gamma>4$,

$$
\|f\|_{\gamma}^{\gamma} \leq\|f\|_{\gamma-4}^{\gamma-4}\|f\|_{\infty}^{4} \leq\|f\|_{\gamma-4}^{\gamma-4}\|f\|_{2}^{2}\left\|D_{+} f\right\|_{2}^{2}
$$

from which we immediately get (4.17) as soon as $\gamma \geq 6$, since in this case $\|f\|_{\gamma-4} \leq\|f\|_{2}$, by the monotonicity properties of $l^{p}(\mathbb{Z})$ norms.

Finally, we come to the

Proof of Theorem 1.2. Assume that $V$ obeys A1 through A3. Except for the finiteness of the threshold $\lambda_{\mathrm{cr}}$, all claims of Theorem 1.2 follow immediately from Theorem 4.1. In addition, Theorem 4.1 shows that the threshold is finite if and only if there exists $f \in l^{2}(\mathbb{Z})$ with

$$
N(f)=\int_{\mathbb{R}} \sum_{x \in \mathbb{Z}} V\left(\left|T_{r} f(x)\right|\right) \mu(d r)>0 .
$$

Lemma B.4 shows that under assumptions A2 and A3 we have $\lim _{a \rightarrow \infty} V(a)=\infty$ and thus $N(f)$ should be large, in particular positive, if $f$ is 'large'. Since $V$ can be negative and due to the nonlocal nature of $N$, this is not obvious, however. Moreover, in the discrete setting there are no nice initial conditions for which one can calculate the time evolution $T_{r} f$ and then also $N(f)$ explicitly, so the proof turns out to be a bit technical. It is deferred to Lemma B.6, where we show that under conditions A1, A2, and A3, there exists a simple family $f_{l} \in l^{2}(\mathbb{Z})$ with $\lim _{l \rightarrow \infty} N\left(f_{l}\right)=\infty$.

If, in addition, we assume $\mathbf{A} 4$, then Lemma B.5 shows $E_{\lambda}^{d_{\mathrm{av}}}<0$ for all $\lambda>0$ and all $d_{\mathrm{av}} \geq 0$. So in this case we have $\lambda_{c r}\left(d_{\mathrm{av}}\right)=0$ for all $d_{\mathrm{av}} \geq 0$.

\section{Exponential DECAY FOR POSITIVE AVERAGE DIFFRACTION}

In this section, we will give the proof of Theorem 1.4. Our strategy for its proof is to first prove some exponential decay and then to boost this to get it up to what the physical heuristic argument in the introduction predicts.

5.1. Some exponential decay. The main goal in this section is to prove

Proposition 5.1. Assume that $V$ obeys the assumption A1. Then any solution $\varphi$ of (1.1) with $\omega<0$ decays exponentially, i.e., there exists $\nu>0$ such that

$$
x \mapsto e^{\nu|x|} \varphi(x) \in l^{2}(\mathbb{Z}) .
$$


To prepare for its proof, define the cutoff function $\chi(s):=\min \left(1,(|s|-1)_{+}\right)$, i.e., $\chi(s)=0$ if $|s| \leq 1, \chi(s)=1$ for $|s| \geq 2$, and linearly interpolating in between. Furthermore, define the functions of $s$ on $\mathbb{R}$

$$
\chi_{\tau}(s):=\chi(s / \tau), \quad F_{\nu, \varepsilon}(s):=\frac{\nu|s|}{1+\varepsilon|s|}, \quad \text { and } \quad \xi_{\nu, \varepsilon, \tau}:=e^{F_{\nu, \varepsilon}} \chi_{\tau},
$$

for any $\tau>0, \nu, \varepsilon \geq 0$.

It is clearly enough to prove that $\xi_{\nu, 0, \tau} \varphi \in l^{2}(\mathbb{Z})$ for some $\nu>0$ and $\tau \geq 1$ for any solution of (1.1) with $\omega<0$. Choosing $g=\xi^{2} \varphi$ in (1.2) and using Lemma 3.6 on has

$$
\begin{aligned}
\omega\|\xi \varphi\|_{2}^{2} & =\operatorname{Re}\left(\omega\left\langle\xi^{2} \varphi, \varphi\right\rangle\right)=D H(\varphi)\left[\xi^{2} \varphi\right]=d_{\mathrm{av}} \operatorname{Re}\left(\left\langle\xi^{2} \varphi,-\Delta \varphi\right\rangle\right)-\operatorname{Re}\left(D N(\varphi)\left[\xi^{2} \varphi\right]\right) \\
& \geq-\frac{d_{\mathrm{av}}}{2}\left\langle\varphi,\left(\left|D_{+} \xi\right|^{2}+\left|D_{-} \xi\right|^{2}\right) \varphi\right\rangle-\operatorname{Re} D N(\varphi)\left[\xi^{2} \varphi\right]
\end{aligned}
$$

where we used the lower bound (C.2) from Lemma C.1 and $\langle\xi \varphi,-\Delta(\xi \varphi)\rangle \geq 0$. Thus for $\omega<0$ we have

$$
\|\xi \varphi\|_{2}^{2} \leq \frac{d_{\mathrm{av}}}{2|\omega|}\left\langle\varphi,\left(\left|D_{+} \xi\right|^{2}+\left|D_{-} \xi\right|^{2}\right) \varphi\right\rangle+\frac{1}{|\omega|} \operatorname{Re} D N(\varphi)\left[\xi^{2} \varphi\right]
$$

which is our starting point for the proof of Proposition 5.1. To use it, the following two Lemmata are helpful.

Lemma 5.2. For all $\nu, \varepsilon \geq 0$ and $\tau>0$ we have

$$
\left|D_{+} \xi_{\nu, \varepsilon, \tau}\right|^{2}+\left|D_{-} \xi_{\nu, \varepsilon, \tau}\right|^{2} \leq \frac{4 e^{2 \nu \tau}}{\tau^{2}}+4\left(e^{\nu}-1\right)^{2} \xi_{\nu, \varepsilon, \tau}^{2}
$$

In particular, for the choice $\nu:=\tau^{-1}$ one has

$$
\left\langle\varphi,\left(\left|D_{+} \xi_{\nu, \varepsilon, \tau}\right|^{2}+\left|D_{-} \xi_{\nu, \varepsilon, \tau}\right|^{2}\right) \varphi\right\rangle \leq \frac{4 e^{2}}{\tau^{2}}\|\varphi\|_{2}^{2}+4\left(e^{\tau^{-1}}-1\right)^{2}\left\|\xi_{\nu, \varepsilon, \tau} \varphi\right\|_{2}^{2}
$$

Proof. Clearly, (5.6) follows from (5.5), so it is enough to prove the first claim. Denoting $\xi=\xi_{\nu, \varepsilon, \tau}$ and $F=F_{\nu, \varepsilon}$, we have

$$
D_{+} \xi(x)=e^{F(x+1)} D_{+} \chi_{\tau}(x)+D_{+} e^{F}(x) \chi_{\tau}(x)
$$

Thus for $x \geq 0$

$$
\begin{aligned}
\left|D_{+} \xi(x)\right|^{2} & \leq 2 e^{2 F(x+1)}\left|D_{+} \chi_{\tau}(x)\right|^{2}+2\left|e^{F(x+1)}-e^{F(x)}\right|^{2} \chi_{\tau}(x)^{2} \\
& \leq 2 e^{2 F(x+1)} \frac{1}{\tau^{2}} \mathbf{1}_{[\tau, 2 \tau-1]}(x)+2\left|e^{F(x+1)-F(x)}-1\right|^{2} \xi(x)^{2} \\
& \leq \frac{2 e^{2 F(2 \tau)}}{\tau^{2}}+2\left(e^{\nu}-1\right)^{2} \xi(x)^{2}
\end{aligned}
$$

where we used $D_{+} \chi_{\tau}(x) \leq \frac{1}{\tau} \mathbf{1}_{[\tau, 2 \tau-1]}(x)$ for $x \geq 0$ in the second inequality, the monotonicity of $F$ and the fact that $F$ obeys the triangle inequality, that is, $F\left(s_{1}+s_{2}\right) \leq F\left(s_{1}\right)+F\left(s_{2}\right)$ for all $s_{1}, s_{2} \in \mathbb{R}$ and hence, by the reverse triangle inequality, also $F(x+1)-F(x) \leq$ $|F(x+1)-F(x)| \leq F(1) \leq \nu$, in the third inequality.

Now let $x \geq 1$. Then

$$
D_{-} \xi(x)=D_{-} e^{F}(x) \chi_{\tau}(x)+e^{F(x-1)} D_{-} \chi_{\tau}(x),
$$


so arguing similarly as above,

$$
\begin{aligned}
\left|D_{-} \xi(x)\right|^{2} & \leq 2\left|D_{-} e^{F}(x) \chi_{\tau}(x)\right|^{2}+2\left|e^{F(x-1)} D_{-} \chi_{\tau}(x)\right|^{2} \\
& \leq 2\left|1-e^{-|F(x)-F(x-1)|}\right|^{2} \xi(x)^{2}+2 e^{2 F(x-1)} \frac{1}{\tau^{2}} \mathbf{1}_{[\tau+1,2 \tau]}(x) \\
& \leq 2\left(e^{\nu}-1\right)^{2} \xi(x)^{2}+\frac{2 e^{2 F(2 \tau)}}{\tau^{2}}
\end{aligned}
$$

for all $x \geq 1$. Using that $\xi$ is symmetric, and hence $D_{+} \xi(-x)=-D_{-} \xi(x)$ holds, the bound (5.7) shows

$$
\left|D_{-} \xi(x)\right|^{2} \leq \frac{2 e^{2 F(2 \tau)}}{\tau^{2}}+2\left(e^{\nu}-1\right)^{2} \xi(x)^{2}
$$

for $x \leq 0$ and the bound (5.8) shows

$$
\left|D_{+} \xi(x)\right|^{2} \leq \frac{2 e^{2 F(2 \tau)}}{\tau^{2}}+2\left(e^{\nu}-1\right)^{2} \xi(x)^{2}
$$

for $x \leq-1$. This proves (5.5).

From (5.4) it is clear that we also have to control $D N(\varphi)\left[\xi^{2} \varphi\right]$. From Lemma 3.6, we get a simple bound

$$
\left|D N\left(f_{2}\right)\left[f_{1}\right]\right| \leq \int_{\mathbb{R}}\left\|T_{r} f_{1} V^{\prime}\left(\left|T_{r} f_{2}\right|\right)\right\|_{1} \mu(d r)
$$

since $\left|\left\langle h_{1}, h_{2}\right\rangle\right| \leq\left\|h_{1} h_{2}\right\|_{1}$. From our assumptions on $V$ one sees

$$
\left|V^{\prime}(a)\right| \lesssim a^{\gamma_{1}-1}+a^{\gamma_{2}-1}
$$

for all $a \in \mathbb{R}_{+}$and therefore

$$
\left|D N\left(f_{2}\right)\left[f_{1}\right]\right| \lesssim L_{\mu}^{\gamma_{1}}\left(f_{1}, f_{2}\right)+L_{\mu}^{\gamma_{2}}\left(f_{1}, f_{2}\right)
$$

where we used

Definition 5.3. For $\gamma \geq 2$ and $\mu$ a finite measure on $\mathbb{R}$ with compact support, let

$$
L_{\mu}^{\gamma}\left(f_{1}, f_{2}\right):=\int_{\mathbb{R}}\left\|T_{r} f_{1}\left|T_{r} f_{2}\right|^{\gamma-1}\right\|_{1} \mu(d r)
$$

A simple bound for the derivative of the nonlocal nonlinearity is given by

Lemma 5.4. Assume that $\mu$ is a finite measure with compact support and $V$ obeys assumption $A$ 1. Then for any $f_{1}, f_{2} \in l^{2}(\mathbb{Z})$

$$
\left|D N\left(f_{2}\right)\left[f_{1}\right]\right| \lesssim\left\|f_{1}\right\|_{2}\left(\left\|f_{2}\right\|_{2}^{\gamma_{1}-1}+\left\|f_{2}\right\|_{2}^{\gamma_{2}-1}\right)
$$

where the implicit constant depends only on $\mu(\mathbb{R})$ and $\operatorname{supp} \mu$ (and the constants in (5.10)). Proof. This follows simply from (5.10)

$$
\left\|T_{r} f_{1}\left|T_{r} f_{2}\right|^{\gamma-1}\right\|_{1} \leq\left\|T_{r} f_{1} T_{r} f_{2}\right\|_{1}\left\|T_{r} f_{2}\right\|_{\infty}^{\gamma-2}
$$

the bound $\left\|T_{r} f_{2}\right\|_{\infty} \leq\left\|T_{r} f_{2}\right\|_{2}=\left\|f_{2}\right\|_{2}$, by unitarity of $T_{r}$, the bound (A.5) and the assumption that $\mu$ is a finite measure with compact support.

We will need a version of Lemma 5.4 which is 'exponentially twisted', 
Lemma 5.5. For $x \in \mathbb{R}$. Then for all $\gamma \geq 2$, all finite measures $\mu$ with compact support, and all $0<\alpha<\frac{1}{2}$

$$
L_{\mu}^{\gamma}\left(e^{F_{\nu, \varepsilon}} h_{1}, e^{-F_{\nu, \varepsilon}} h_{2}\right) \lesssim \min \left(1, s^{-\alpha s}\right)\left\|h_{1}\right\|_{2}\left\|h_{2}\right\|_{2}\left\|e^{-F_{\nu, \varepsilon}} h_{2}\right\|_{2}^{\gamma-2}
$$

where $s:=\operatorname{dist}\left(\operatorname{supp} h_{1}, \operatorname{supp} h_{2}\right) \geq 0$ and the implicit constant is independent of $\varepsilon>0$ and depends increasingly on $\nu \geq 0, \mu(\mathbb{R})$, and the support of $\mu$ and $\alpha$.

Remark 5.6. In the equation above, we set $0^{-\alpha 0}:=\lim _{s \rightarrow 0} s^{-\alpha s}=1$, when $s=0$.

Proof. Let $B>0$ such that $\operatorname{supp} \mu \subset[-B, B]$. Then

$$
L_{\mu}^{\gamma}\left(e^{F_{\nu, \varepsilon}} h_{1}, e^{-F_{\nu, \varepsilon}} h_{2}\right) \leq \mu(\mathbb{R}) \sup _{|r| \leq B}\left\|T_{r}\left(e^{F_{\nu, \varepsilon}} h_{1}\right)\left|T_{r}\left(e^{-F_{\nu, \varepsilon}} h_{2}\right)\right|^{\gamma-1}\right\|_{1}
$$

Fix $r \in \mathbb{R}$, then

$$
\left\|T_{r}\left(e^{F_{\nu, \varepsilon}} h_{1}\right)\left|T_{r}\left(e^{-F_{\nu, \varepsilon}} h_{2}\right)\right|^{\gamma-1}\right\|_{1} \leq\left\|T_{r}\left(e^{F_{\nu, \varepsilon}} h_{1}\right) T_{r}\left(e^{-F_{\nu, \varepsilon}} h_{2}\right)\right\|_{1}\left\|T_{r}\left(e^{-F_{\nu, \varepsilon}} h_{2}\right)\right\|_{\infty}^{\gamma-2}
$$

The first factor is bounded by (A.6) and for the second factor we simply note

$$
\left\|T_{r}\left(e^{-F_{\nu, \varepsilon}} h_{2}\right)\right\|_{\infty} \leq\left\|T_{r}\left(e^{-F_{\nu, \varepsilon}} h_{2}\right)\right\|_{2}=\left\|e^{-F_{\nu, \varepsilon}} h_{2}\right\|_{2}
$$

since $T_{r}$ is unitary on $l^{2}(\mathbb{Z})$. Since $\frac{2\left(4 B e^{\nu}\right)^{\left\lceil\frac{s}{2}\right\rceil}}{\left\lceil\frac{s}{2}\right\rceil !} \lesssim s^{-\alpha s}$ for any fixed $B, \nu>0,0<\alpha<\frac{1}{2}$, and all bounded $\nu$, this finishes the proof.

A useful consequence of this is

Corollary 5.7. Assume that $V$ obeys assumption A1. Then for the choice $\nu=\tau^{-1}$

$$
\left|D N(\varphi)\left[\xi_{\nu, \varepsilon, \tau}^{2} \varphi\right]\right| \lesssim o(1)\left(\left\|\xi_{\nu, \varepsilon, \tau} \varphi\right\|_{2}^{2}+\left\|\xi_{\nu, \varepsilon, \tau} \varphi\right\|_{2}\right)
$$

where the implicit constant depends only on $\gamma_{1}, \gamma_{2}, \mu(\mathbb{R})$, the support of $\mu$, $\|\varphi\|_{2}$ and $o(1)$ denotes a term which, for fixed $\varphi \in l^{2}(\mathbb{Z})$, goes to zero uniformly in $\varepsilon>0$ as $\tau \rightarrow \infty$.

Proof. Set $\xi=\xi_{\nu, \varepsilon, \tau}$ and $F=F_{\nu, \varepsilon}$. Because of (5.10), we need to control $L_{\mu}^{\gamma}\left(\xi^{2} \varphi, \varphi\right)$ for $\gamma=\gamma_{1}$ and $\gamma=\gamma_{2}$. Let $\varphi_{\tau}:=\chi_{\tau} \varphi$ and $h_{\tau}:=e^{F} \varphi_{\tau}$ and split $h:=e^{F} \varphi$ into $h_{\tau}$ and $h_{\leq \tau}:=\left(1-\chi_{\tau}\right) h$. Then $h=h_{\tau}+h_{\leq \tau}$ and since $|a+b|^{\gamma-1} \lesssim|a|^{\gamma-1}+|b|^{\gamma-1}$, we have

$$
\begin{aligned}
L_{\mu}^{\gamma}\left(\xi^{2} \varphi, \varphi\right) & =L_{\mu}^{\gamma}\left(e^{F} \chi_{\tau} h_{\tau}, e^{-F} h\right) \\
& \lesssim L_{\mu}^{\gamma}\left(e^{F} \chi_{\tau} h_{\tau}, e^{-F} h_{\tau}\right)+L_{\mu}^{\gamma}\left(e^{F} \chi_{\tau} h_{\tau}, e^{-F} h_{\leq \tau}\right)
\end{aligned}
$$

Lemma 5.5 yields

$$
L_{\mu}^{\gamma}\left(e^{F} \chi_{\tau} h_{\tau}, e^{-F} h_{\tau}\right) \lesssim\left\|h_{\tau}\right\|_{2}^{2}\left\|\varphi_{\tau}\right\|_{2}^{\gamma-2}
$$

since $\left\|\chi_{\tau} h_{\tau}\right\|_{2} \leq\left\|h_{\tau}\right\|_{2}$. Splitting $h_{\leq \tau}=h_{\ll \tau}+h_{\sim \tau}$, where $h_{\ll \tau}:=\mathbf{1}_{[-\tau / 2, \tau / 2]}(x) h_{\tau}$ and $h_{\sim \tau}:=h_{\tau}-h_{\ll \tau}$ we also have

$$
\begin{aligned}
L_{\mu}^{\gamma}\left(e^{F} \chi_{\tau} h_{\tau}, e^{-F} h_{\leq \tau}\right) & \lesssim L_{\mu}^{\gamma}\left(e^{F} \chi_{\tau} h_{\tau}, e^{-F} h_{\ll \tau}\right)+L_{\mu}^{\gamma}\left(e^{F} \chi_{\tau} h_{\tau}, e^{-F} h_{\sim \tau}\right) \\
& \leq(\tau / 2)^{-\tau / 8}\left\|h_{\tau}\right\|_{2}\left\|h_{\ll \tau}\right\|_{2}\left\|\varphi_{\ll \tau}\right\|_{2}^{\gamma-2}+\left\|h_{\tau}\right\|_{2}\left\|h_{\sim \tau}\right\|_{2}\left\|\varphi_{\sim \tau}\right\|_{2}^{\gamma-2} \\
& \leq(\tau / 2)^{-\tau / 8} e^{1 / 2}\left\|h_{\tau}\right\|_{2}\|\varphi\|_{2}^{\gamma-1}+e^{2}\left\|h_{\tau}\right\|_{2}\left\|\varphi_{\sim \tau}\right\|_{2}^{\gamma-1}
\end{aligned}
$$

because of Lemma 5.5, since $h_{\ll \tau}$ and $h_{\tau}$ have supports separated by at least $\tau / 2$ and $\left\|h_{\ll \tau}\right\|_{2} \leq e^{\nu \tau / 2}\left\|\varphi_{\ll \tau}\right\|_{2} \leq e^{1 / 2}\|\varphi\|_{2}$ and $\left\|h_{\sim \tau}\right\|_{2} \leq e^{2 \nu \tau}\left\|\varphi_{\sim \tau}\right\|_{2} \leq e^{2}\left\|\varphi_{\sim \tau}\right\|_{2}$. Together, the above bounds show

$$
L_{\mu}^{\gamma}\left(\xi^{2} \varphi, \varphi\right) \lesssim\left(\left\|\varphi_{\tau}\right\|^{\gamma-2}+(\tau / 2)^{-\tau / 8}\|\varphi\|_{2}^{\gamma-1}+\left\|\varphi_{\sim \tau}\right\|_{2}^{\gamma-1}\right)\left(\left\|h_{\tau}\right\|_{2}^{2}+\left\|h_{\tau}\right\|_{2}\right) .
$$

Since, for fixed $\varphi \in l^{2}(\mathbb{Z})$, the term $\left\|\varphi_{\tau}\right\|_{2}^{\gamma-2}+(\tau / 2)^{-\tau / 8}\|\varphi\|_{2}^{\gamma-1}+\left\|\varphi_{\sim \tau}\right\|_{2}^{\gamma-1}$ goes to zero as $\tau \rightarrow \infty$, this finishes the proof of the corollary. 
Now we can give the

Proof of Proposition 5.1. Let $\varphi$ be a solution of (1.1) with $\omega<0$ and $d_{\mathrm{av}}>0$. Then with $\chi_{\tau}, F_{\nu, \varepsilon}$, and $\xi_{\nu, \varepsilon, \tau}$ as before together with the choice $\nu=\tau^{-1}$, the inequality (5.4) and Lemma 5.2 and Corollary 5.7 show

$$
\left\|\xi_{\nu, \varepsilon, \tau} \varphi\right\|_{2}^{2} \leq o_{1}(1)\left(\left\|\xi_{\nu, \varepsilon, \tau} \varphi\right\|_{2}^{2}+\left\|\xi_{\nu, \varepsilon, \tau} \varphi\right\|_{2}\right)+o_{2}(1)
$$

where $o_{1}(1)$ and $o_{2}(1)$ denote terms which, for fixed $\varphi \in l^{2}(\mathbb{Z})$ and $\nu=\tau^{-1}$, go to zero as $\tau \rightarrow \infty$ uniformly in $\varepsilon>0$. Choosing $\tau$ so large that $o_{1}(1) \leq \frac{1}{2}$, the bound (5.13) gives

$$
\left\|\xi_{\nu, \varepsilon, \tau} \varphi\right\|_{2}^{2}-\left\|\xi_{\nu, \varepsilon, \tau} \varphi\right\|_{2} \lesssim 1
$$

as long as $\nu=\tau^{-1}$ and $\tau$ is large enough. Clearly, (5.14) shows that $\left\|\xi_{\nu, \varepsilon, \tau} \varphi\right\|_{2}$ stays bounded as $\varepsilon \rightarrow 0$, so

$$
\left\|e^{F_{\nu, 0}} \varphi_{\tau}\right\|_{2}=\lim _{\varepsilon \rightarrow 0}\left\|e^{F_{\nu, \varepsilon}} \varphi_{\tau}\right\|_{2}<\infty
$$

as long as $\nu=\tau^{-1}$ and $\tau$ is large enough.

5.2. Boosting the decay rate. Given Proposition 5.1 we know that a solution $\varphi$ of (1.1) with $\omega<0$ and $d_{\mathrm{av}}>0$ has some exponential decay, that is, for some $\nu>0$ we have $e^{\nu|\cdot|} \varphi(\cdot) \in l^{2}(\mathbb{Z})$. The goal in this section is to boost this to prove the lower bound (1.11) on the exponential decay rate from Theorem 1.4. For this we need a refinement of (5.4) and of Lemma 5.2. Looking at the proof of (5.4), we need to refine the error in the IMS localization formula. This is the context of

Lemma 5.8. Let $F: \mathbb{Z} \rightarrow \mathbb{R}$ be bounded. Then for all $\varphi \in l^{2}(\mathbb{Z})$

$$
\operatorname{Re}\left(\left\langle e^{2 F} \varphi,-\Delta \varphi\right\rangle\right) \geq-\left\langle e^{F} \varphi,\left(\cosh \left(D_{+} F\right)+\cosh \left(D_{-} F\right)-2\right) e^{F} \varphi\right\rangle
$$

Proof. Using the formula (C.1) for $\xi=e^{F}$ one sees

$$
\begin{aligned}
\operatorname{Re}\left(\left\langle e^{2 F} \varphi,-\Delta \varphi\right\rangle\right) & =\left\langle e^{F} \varphi,-\Delta\left(e^{F} \varphi\right)\right\rangle-\sum_{x \in \mathbb{Z}}\left|D_{+} e^{F}(x)\right|^{2} \operatorname{Re}(\overline{\varphi(x)} \varphi(x+1)) \\
& \geq-\sum_{x \in \mathbb{Z}}\left|D_{+} e^{F}(x)\right|^{2} \operatorname{Re}(\overline{\varphi(x)} \varphi(x+1))
\end{aligned}
$$

since $\left\langle e^{F} \varphi,-\Delta\left(e^{F} \varphi\right)\right\rangle \geq 0$. A simple calculation shows

$$
\left|D_{+} e^{F}(x)\right|^{2}=2(\cosh (F(x+1)-F(x))-1) e^{F(x)} e^{F(x+1)} \text {. }
$$

Thus

$$
\begin{aligned}
\sum_{x \in \mathbb{Z}}\left|D_{+} e^{F}(x)\right|^{2} \operatorname{Re}(\overline{\varphi(x)} \varphi(x+1)) \\
\quad=\sum_{x \in \mathbb{Z}}\left(\cosh \left(D_{+} F(x)\right)-1\right) 2 \operatorname{Re}\left(e^{F(x)} \overline{\varphi(x)} e^{F(x+1)} \varphi(x+1)\right) \\
\leq \sum_{x \in \mathbb{Z}}\left(\cosh \left(D_{+} F(x)\right)-1\right)\left(\left|e^{F(x)} \varphi(x)\right|^{2}+\left|e^{F(x+1)} \varphi(x+1)\right|^{2}\right) \\
\quad=\left\langle e^{F} \varphi,\left(\cosh \left(D_{+} F\right)+\cosh \left(D_{-} F\right)-2\right) e^{F} \varphi\right\rangle
\end{aligned}
$$


Since cosh is even and increasing on $\mathbb{R}_{+}$and

$$
\left|D_{ \pm} F_{\nu, \varepsilon}(x)\right|=\left|F_{\nu, \varepsilon}(x \pm 1)-F_{\nu, \varepsilon}(x)\right| \leq F_{\nu, \varepsilon}(1) \leq \nu
$$

Lemma 5.8 gives for $F=F_{\nu, \varepsilon}$ and any solution $\varphi$ of (1.1) with $\omega<0$ and $d_{\mathrm{av}}>0$ the bound

$$
\begin{aligned}
\omega\left\|e^{F} \varphi\right\|_{2}^{2} & =\operatorname{Re}\left(\omega\left\langle e^{2 F} \varphi, \varphi\right\rangle\right)=d_{\mathrm{av}} \operatorname{Re}\left(\left\langle e^{2 F} \varphi,-\Delta \varphi\right\rangle\right)-\operatorname{Re}\left(D N(\varphi)\left[e^{2 F} \varphi\right]\right) \\
& \geq-d_{\mathrm{av}}\left\langle e^{F} \varphi, 2(\cosh (\nu)-1) e^{F} \varphi\right\rangle-\left|D N(\varphi)\left[e^{2 F} \varphi\right]\right|
\end{aligned}
$$

In other words, since $\omega<0$, we have the bound

$$
\left(|\omega|-2 d_{\mathrm{av}}(\cosh (\nu)-1)\right)\left\|e^{F_{\nu, \varepsilon}} \varphi\right\|_{2}^{2} \leq\left|D N(\varphi)\left[e^{2 F_{\nu, \varepsilon}} \varphi\right]\right|
$$

which will help to control $\left\|e^{F_{\nu, \varepsilon}} \varphi\right\|$ as long as $|\omega|>2 d_{\mathrm{av}}(\cosh (\nu)-1)$. To control the right hand side of (5.16), we note

Lemma 5.9. Assume that $V$ obeys the assumption $A 1$ and $\mu$ is a finite measure with compact support. Then, if $e^{\nu_{0}|\cdot|} \varphi(\cdot) \in l^{2}(\mathbb{Z})$ for some $\nu_{0}>0$ we have

$$
\limsup _{\varepsilon \rightarrow 0}\left|D N(\varphi)\left[e^{2 F_{\nu, \varepsilon}} \varphi\right]\right|<\infty
$$

for all $0<\nu \leq \frac{\gamma_{1}}{2} \nu_{0}$.

Remark 5.10. One might hope that even despite the nonlocal nature of $D N$ one could have

$$
\left.\left|D N(\varphi)\left[e^{F} \psi\right]\right| \lesssim \mid D N\left(e^{F} \varphi\right) \psi\right] \mid
$$

Setting $\psi_{\varepsilon}:=e^{F_{\nu_{0}, \varepsilon}} \varphi$ and using $2 F_{\nu, \varepsilon}=F_{2\left(\nu-\nu_{0}\right), \varepsilon}+2 F_{\nu_{0}, \varepsilon}$, one could conclude from this

$$
\left|D N(\varphi)\left[e^{2 F_{\nu, \varepsilon}} \varphi\right]\right|=\left|D N(\varphi)\left[e^{F_{\nu_{0}, \varepsilon}} e^{F_{2\left(\nu-\nu_{0}\right), \varepsilon}} \psi_{\varepsilon}\right]\right| \lesssim\left|D N\left(\psi_{\varepsilon}\right)\left[e^{F_{2\left(\nu-\nu_{0}\right), \varepsilon}} \psi_{\varepsilon}\right]\right|
$$

but since $\gamma_{1}>2$, we have $\nu-\nu_{0}>0$ for $\nu_{0}<\nu \leq \frac{\gamma_{1}}{2} \nu_{0}$ and this leaves an excess exponential weight $F_{2\left(\nu-\nu_{0}\right), \varepsilon}$. The point of the Lemma is that this excess weight is absorbed by the nonlinearity even though it is nonlocal.

Proof. Set $\psi_{\varepsilon}:=e^{F_{\nu_{0}, \varepsilon}} \varphi$. Then $\lim \sup _{\varepsilon \rightarrow 0}\left\|\psi_{\varepsilon}\right\|_{2}<\infty$ and with (5.10) and Definition 5.3 we have

$$
\left|D N(\varphi)\left[e^{2 F_{\nu, \varepsilon}} \varphi\right]\right|=\left|D N(\varphi)\left[e^{F_{2 \nu-\nu_{0}, \varepsilon}} \psi_{\varepsilon}\right]\right| \lesssim L_{\mu}^{\gamma_{1}}\left(e^{F_{2 \nu-\nu_{0}, \varepsilon}} \psi_{\varepsilon}, \varphi\right)+L_{\mu}^{\gamma_{2}}\left(e^{F_{2 \nu-\nu_{0}, \varepsilon}} \psi_{\varepsilon}, \varphi\right)
$$

Using (A.7), we see

$$
\begin{aligned}
L_{\mu}^{\gamma}\left(e^{F_{2 \nu-\nu_{0}, \varepsilon}} \psi_{\varepsilon}, \varphi\right) & \leq \mu(\mathbb{R}) \sup _{|r| \leq B}\left\|T_{r}\left(e^{F_{2 \nu-\nu_{0}, \varepsilon}} \psi_{\varepsilon}\right)\left|T_{r} \varphi\right|^{\gamma-1}\right\|_{1} \\
& \leq \mu(\mathbb{R})\left(2 e^{4 B\left(1+e^{\nu}\right)}\right)^{\gamma}\left\|\psi_{\varepsilon}\right\|_{2}\left\|e^{F_{\left(2 \nu-\nu_{0}\right) /(\gamma-1), \varepsilon}} \varphi\right\|_{2}^{\gamma-1}
\end{aligned}
$$

for $\gamma=\gamma_{1}, \gamma_{2}$. By assumption, $\lim _{\sup _{\varepsilon \rightarrow 0}}\left\|\psi_{\varepsilon}\right\|_{2}<\infty$ and in order to have

$$
\limsup _{\varepsilon \rightarrow 0}\left\|e^{F_{\left(2 \nu-\nu_{0}\right) /(\gamma-1), \varepsilon}} \varphi\right\|_{2}<\infty
$$

we need $2 \nu-\nu_{0} \leq\left(\gamma_{1}-1\right) \nu_{0}$, which is equivalent to $\nu \leq \frac{\gamma_{1}}{2} \nu_{0}$, so (5.17) follows.

Before we come our key result for boosting the exponential decay rate, we need some more notation. Note that $0 \leq \nu \mapsto 2 d_{\text {av }}(\cosh (\nu)-1)$ is strictly increasing from zero to infinity. Thus for any $\omega<0$ there exist a unique $\bar{\nu}>0$ such that

$$
2 d_{\mathrm{av}}(\cosh (\bar{\nu})-1)=|\omega| \text {. }
$$

In other words, $\bar{\nu}$ is given by the right hand side of (1.11). 
Proposition 5.11 (Boosting the exponential decay rate). Assume that $V$ obeys the assumption $A 1$ and that $\varphi$ is a solution of (1.1) for some $\omega<0$ and $d_{\mathrm{av}}>0$, and $\bar{\nu}$ is given by (5.18). Furthermore, assume that for some $0<\nu<\bar{\nu}$ we have $e^{\nu|\cdot|} \varphi \in l^{2}(\mathbb{Z})$. If $\delta>0$ is such that

$$
\nu+\delta<\bar{\nu} \quad \text { and } \quad \delta \leq \frac{\gamma_{1}-2}{2} \nu
$$

then $e^{(\nu+\delta)|\cdot|} \varphi \in l^{2}(\mathbb{Z})$.

Proof. Let $\nu_{1}:=\nu+\delta<\bar{\nu}$. Then $|\omega|-2 d_{\mathrm{av}}\left(\cosh \left(\nu_{1}\right)-1\right)>0$ and (5.16) shows

$$
\left\|e^{F_{\nu_{1}, \varepsilon}} \varphi\right\|_{2}^{2} \lesssim\left|D N(\varphi)\left[e^{2 F_{\nu_{1}, \varepsilon}} \varphi\right]\right| \text {. }
$$

Since the condition $\delta \leq \frac{\gamma_{1}-2}{2} \nu$ is equivalent to $\nu_{1} \leq \frac{\gamma_{1}}{2} \nu$, (5.19), Lemma 5.9, and the monotone convergence theorem yield

$$
\left\|e^{F_{\nu_{1}, 0}} \varphi\right\|_{2}^{2}=\lim _{\varepsilon \rightarrow 0}\left\|e^{F_{\nu_{1}, \varepsilon}} \varphi\right\|_{2}^{2} \leq \limsup _{\varepsilon \rightarrow 0}\left|D N(\varphi)\left[e^{2 F_{\nu_{1}, \varepsilon}} \varphi\right]\right|<\infty
$$

which proves the claim.

Now we come to the

Proof of Theorem 1.4. From Proposition 5.1 we know that

$$
\nu_{*}:=\sup \left\{\nu>0 \mid\left(x \mapsto e^{\nu|x|} \varphi(x)\right) \in l^{2}(\mathbb{Z})\right\}>0 .
$$

In order to prove the lower bound (1.11), let us assume that, in the contrary, $0<\nu_{*}<\bar{\nu}$, where $\bar{\nu}$ is given by (5.18). Take any $0<\nu_{0}<\nu_{*}$ and choose

$$
\delta=\delta_{\nu_{0}}:=\min \left(\frac{\bar{\nu}-\nu_{0}}{2}, \frac{\gamma_{1}-2}{2} \nu_{0}\right) .
$$

Then Proposition 5.11 shows $e^{\nu|\cdot|} \varphi \in l^{2}(\mathbb{Z})$ for $\nu=\nu_{0}+\delta_{\nu_{0}}$, that is,

$$
\nu_{0}+\delta_{\nu_{0}} \leq \nu_{*} \text { for any } 0<\nu_{0}<\nu_{*}
$$

by the definition of $\nu_{*}$. However, since we assumed $0<\nu_{*}<\bar{\nu}$ and $\gamma_{1}>2$ we have $\frac{\bar{\nu}+\nu_{*}}{2}>\nu_{*}$ and $\frac{\gamma_{1}}{2} \nu_{*}>\nu_{*}$. Thus

$$
\nu_{0}+\delta_{\nu_{0}}=\min \left(\frac{\bar{\nu}+\nu_{0}}{2}, \frac{\gamma_{1}}{2} \nu_{0}\right) \rightarrow \min \left(\frac{\bar{\nu}+\nu_{*}}{2}, \frac{\gamma_{1}}{2} \nu_{*}\right)>\nu_{*} \quad \text { as } \nu_{0} \nearrow \nu_{*}
$$

which is a contradiction. So $\nu_{*} \geq \bar{\nu}$.

\section{Super-EXPonential DECAY FOR ZERo AVERAGE DiffraCtion}

In this section, we show that any solution $\varphi \in l^{2}(\mathbb{Z})$ of $(1.1)$ for zero average diffraction decays super-exponentially, with an explicit lower bound on the decay rate. We are guided by the approach of [16] and follow in part their argument, however, we also need to make substantial modifications. Similar to [16], we focus on the tail distribution $\beta$ of $\varphi$, where

$$
\beta(n):=\left(\sum_{|x| \geq n}|\varphi(x)|^{2}\right)^{1 / 2}
$$

for $n \in \mathbb{N}_{0}$. Our main tool for showing this very fast decay is the following self-consistency bound on the tail distribution $\beta$, which generalizes the one in [16]. This bound will be important for establishing some super-expoential decay in Section 6.1, as well as boosting it to the lower bound in Section 6.2, which together will yield the proof of Theorem 1.7. 
Proposition 6.1 (Self-consistency bound). Assume that $V$ obeys the conditions of assumption $\boldsymbol{A} 1$ and $\omega \neq 0$. If $\varphi$ is a solution of (1.1) for $d_{\mathrm{av}}=0$ then with $\theta:=\gamma_{1}-1>1$ and for any $m, n \in \mathbb{N}_{0}$ and $0<\alpha<\frac{1}{2}$ the bound

$$
\beta(n+m) \lesssim \beta(n)^{\theta}+(m+1)^{-\alpha(m+1)}
$$

holds where the implicit constant depends only on $\alpha, \omega$, and $\|\varphi\|_{2}$.

Proof. If $\varphi$ is a solution of (1.1) with $\omega \neq 0$ and $d_{\mathrm{av}}=0$, then

$$
\langle\varphi, g\rangle=-\omega^{-1} D N(\varphi)[g]
$$

with $D N(\varphi)[g]$ from Remark 3.7. Now define the hard cutoff $\chi_{l}(x):=1$ if $|x| \geq l$ and $\chi_{l}(x)=0$ if $|x| \leq l-1$ and choose $g=\varphi_{\gg}:=\chi_{l} \varphi$ with $l=n+m$. Then (5.10) again shows

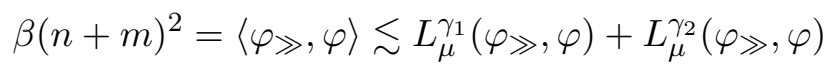

and splitting $\varphi=\varphi_{>}+\varphi_{<}$with $\varphi_{>}:=\chi_{n} \varphi$ and $\varphi_{<}:=\varphi-\varphi_{>}$, which has support in $[-(n-1), n-1]$ shows

$$
\begin{aligned}
\beta(n+m)^{2} & \lesssim L_{\mu}^{\gamma_{1}}\left(\varphi_{\gg}, \varphi\right)+L_{\mu}^{\gamma_{2}}\left(\varphi_{\gg}, \varphi\right) \\
& \lesssim L_{\mu}^{\gamma_{1}}\left(\varphi_{\gg}, \varphi_{>}\right)+L_{\mu}^{\gamma_{2}}\left(\varphi_{\gg}, \varphi_{>}\right)+L_{\mu}^{\gamma_{1}}\left(\varphi_{\gg}, \varphi_{<}\right)+L_{\mu}^{\gamma_{2}}\left(\varphi_{\gg}, \varphi_{<}\right) .
\end{aligned}
$$

Lemma 5.5 for $F=F_{\nu, \varepsilon}=0$ shows

$$
L_{\mu}^{\gamma}\left(\varphi_{\gg}, \varphi_{>}\right) \lesssim\left\|\varphi_{\gg}\right\|_{2}\left\|\varphi_{>}\right\|_{2}^{\gamma-1}=\beta(n+m) \beta(n)^{\gamma-1}
$$

and

$$
L_{\mu}^{\gamma}\left(\varphi_{\gg}, \varphi_{<}\right) \lesssim(m+1)^{-\alpha(m+1)}\left\|\varphi_{\gg}\right\|_{2}\left\|\varphi_{<}\right\|_{2}^{\gamma-1} \leq(m+1)^{-\alpha(m+1)} \beta(n+m) \beta(0)^{\gamma-1}
$$

for $\gamma=\gamma_{1}, \gamma_{2}$. Since $\beta(n)^{\gamma_{2}-1} \leq \beta(0)^{\gamma_{2}-\gamma_{1}} \beta(n)^{\gamma_{1}-1}$, this finishes the proof.

The self-consistency bound from Proposition 6.1 is our main tool to prove Theorem 1.7. Again, we split the argument, first we show some super-exponential decay and then we boost this. The first part is, with considerable changes, similar to the approach in [16], but since the decay rate of Theorem 1.7 for $V(a) \sim|a|^{2} a$ is quite a bit better than in [16], we have to do much better in the second step.

\subsection{Some super-exponential decay.}

Proposition 6.2 (Some super-exponential decay). Let $\beta$ be a decreasing non-negative function, vanishing at infinity, which obeys the self-consistency bound (6.2) of Proposition 6.1 for some $\theta=\gamma_{1}-1>1$. Then there exists $\nu>0$ such that

$$
\beta(n) \lesssim(n+1)^{-\nu(n+1)} \quad \text { for all } n \in \mathbb{N}_{0} .
$$

Corollary 6.3 (= first step in the proof of Theorem 1.7). Assume $d_{\mathrm{av}}=0$ and $V$ obeys assumption $A 1$. Then for any solution $\varphi$ of (1.1) with $\omega \neq 0$, there exists $\nu>0$ such that

$$
|\varphi(x)| \lesssim(|x|+1)^{-\nu(|x|+1)}
$$

for all $x \in \mathbb{Z}$.

Proof. Given Proposition 6.2, this follows immediately from $|\varphi(x)| \leq \beta(|x|)$, where $\beta$ is defined in (6.1). 
In order to prove Proposition 6.2 , for any $\nu \geq 0$, define the weight $H_{\nu}$ by

$$
H_{\nu}(s):=(s+1)^{\nu(s+1)}
$$

for $s \geq 0$ and its regularized version of $H_{\nu, \varepsilon}$ given by

$$
H_{\nu, \varepsilon}(s):=\frac{H_{\nu}(s)}{1+\varepsilon H_{\nu}(s)}=\frac{1}{H_{\nu}(s)^{-1}+\varepsilon} .
$$

for $s, \varepsilon \geq 0$. We need some basic properties of $H_{\nu, \varepsilon}$ given in

Lemma 6.4. (i) For any $\varepsilon \geq 0$, the function $(\nu, s) \in \mathbb{R}^{+} \times \mathbb{R}^{+} \mapsto H_{\nu, \varepsilon}(s)$ is bounded above by $\varepsilon^{-1}$. Moreover, the function $H_{\nu, \varepsilon}(s)$ is increasing in $s, \nu \geq 0$, decreasing in $\varepsilon \geq 0$, and depends continuously on $\nu, \varepsilon, s \geq 0$.

(ii) Let $0<\sigma<1$ and $0<\nu<\frac{\sigma}{8}$. Furthermore ${ }^{4}$, let $m:=\lfloor\sigma(l+1)\rfloor$ for $l \in \mathbb{N}_{0}$. Then

$$
H_{\nu}(l)(m+1)^{-\frac{m+1}{4}} \leq \exp \left(-\left(\frac{\sigma}{8} \ln (l+1)+\frac{1}{4 e}\right)(l+1)\right) .
$$

(iii) Let $\nu \geq 0, \theta>1,0<\sigma<\frac{\theta-1}{\theta}$, then there exist a constant $C=C(\theta, \sigma, \nu)$ which is decreasing in $\theta$, increasing in $\sigma$ and $\nu$, such that with $n:=l-\lfloor\sigma(l+1)\rfloor$

$$
H_{\nu, \varepsilon}(l) \leq C H_{\nu, \varepsilon}(n)^{\theta}
$$

for all $l \in \mathbb{N}_{0}$ and $0 \leq \varepsilon \leq 1$.

(iv) Let $\beta: \mathbb{N}_{0} \rightarrow \mathbb{R}$ be a bounded function and $\tau \in \mathbb{N}_{0}$. Then the map

$$
[0, \infty) \times(0, \infty) \ni(\nu, \varepsilon) \mapsto\|\beta\|_{\nu, \varepsilon, \tau}:=\sup _{l \geq \tau} H_{\nu, \varepsilon}(l) \beta(l)
$$

is continuous.

(v) For $0<\nu, \tau \in \mathbb{N}_{0}$, and an arbitrary bounded function $\beta: \mathbb{N}_{0} \rightarrow \mathbb{R}$

$$
\|\beta\|_{\nu, 0, \tau}=\lim _{\varepsilon \rightarrow 0}\|\beta\|_{\nu, \varepsilon, \tau}=\sup _{0<\varepsilon \leq 1}\|\beta\|_{\nu, \varepsilon, \tau} .
$$

We will give the proof of this Lemma at the end of this section and come to the

Proof of Proposition 6.2. We need to show that for some $\nu>0$ and some $\tau \in \mathbb{N}$

$$
\sup _{l \geq \tau} H_{\nu}(l) \beta(l)<\infty .
$$

Let $\alpha=\frac{1}{4}$ and $\theta=\gamma_{1}-1>1$. The self-consistency bound (6.2) shows

$$
\beta(l) \lesssim \beta(n)^{\theta}+(m+1)^{-(m+1) / 4}
$$

for all $l, m, n \in \mathbb{N}_{0}$ with $l=n+m$.

We fix $\sigma=\frac{\theta-1}{2 \theta}$ then $0<\sigma<1 / 2$ and we consider $0<\nu \leq \sigma / 8$, which we choose more precisely below, and let

$$
m=\lfloor\sigma(l+1)\rfloor .
$$

Multiplying (6.9) by $H_{\nu, \varepsilon}(l)$ and using Lemma (iii) shows

$$
H_{\nu, \varepsilon}(l) \beta(l) \lesssim\left(H_{\nu, \varepsilon}(n) \beta(n)\right)^{\theta}+H_{\nu}(l)(m+1)^{-(m+1) / 4}
$$

uniformly in $0<\varepsilon \leq 1$, hence, for any $\tau \in \mathbb{N}_{0}$, since $n=l-m \geq\lfloor(1-\sigma)(\tau+1)-1\rfloor$ if $l \geq \tau$, also

$$
\|\beta\|_{\nu, \varepsilon, \tau}=\sup _{l \geq \tau} H_{\nu, \varepsilon}(l) \beta(l) \lesssim\|\beta\|_{\nu, \varepsilon, \tilde{\tau}}^{\theta}+\sup _{l \geq \tau} H_{\nu}(l)(m+1)^{-(m+1) / 4}
$$

\footnotetext{
${ }^{4}$ Recall $\lfloor s\rfloor:=\max \{k \in \mathbb{Z} \mid k \leq s\}$
} 
where we introduced $\tilde{\tau}:=\lfloor(1-\sigma)(\tau+1)-1\rfloor$. Note

$$
\begin{aligned}
\|\beta\|_{\nu, \varepsilon, \tilde{\tau}}^{\theta} & \leq\|\beta\|_{\nu, \varepsilon, \tau}^{\theta}+\max _{\tilde{\tau} \leq n \leq \tau-1}\left(H_{\nu, \varepsilon}(n) \beta(n)\right)^{\theta} \leq\|\beta\|_{\nu, \varepsilon, \tau}^{\theta}+H_{\nu}(\tau-1)^{\theta} \beta(\tilde{\tau})^{\theta} \\
& =\|\beta\|_{\nu, \varepsilon, \tau}^{\theta}+\tau^{\theta \nu \tau} \beta(\tilde{\tau})^{\theta}
\end{aligned}
$$

by the monotonicity of $H_{\nu, \varepsilon}$ and $\beta$. So setting $R_{1}(\tau):=\sup _{l \geq \tau} e^{-\left[\frac{\sigma}{8} \ln (l+1)-\frac{1}{4 e}\right](l+1)}$, using Lemma (ii) and $0<\nu \leq \frac{\sigma}{8}$, we arrive at

$$
\|\beta\|_{\nu, \varepsilon, \tau} \leq C\left(\|\beta\|_{\nu, \varepsilon, \tau}^{\theta}+\tau^{\theta \nu \tau} \beta(\tilde{\tau})^{\theta}+R_{1}(\tau)\right)
$$

for some universal constant $C$ independent of $\tau \in \mathbb{N}$. Now let $\tau_{1}$ be so large that $\frac{1}{\tau \ln \tau} \leq \frac{\sigma}{8}$ for all $\tau \geq \tau_{1}$. Choosing $\nu:=\frac{1}{\tau \ln \tau}$ gives $\tau^{\theta \nu \tau}=e^{\theta}$ and with

$$
G(u):=u-C u^{\theta} \quad \text { for } u \geq 0
$$

we see that (6.10) can be rewritten as

$$
G\left(\|\beta\|_{\nu, \varepsilon, \tau}\right) \leq R_{2}(\tau)
$$

for all $\tau \geq \tau_{1}$, where $\nu=\frac{1}{\tau \ln \tau}$ and $R_{2}(\tau):=C\left(e^{\theta} \beta(\tilde{\tau})+R_{1}(\tau)\right)$ with $\tilde{\tau}:=\lfloor(1-\sigma)(\tau+1)-1\rfloor$.

Now the argument continues exactly as in [16], we will give it for the convenience of the reader: Certainly $G$ is continuous on $[0, \infty)$ with $G(0)=0$ and $\lim _{u \rightarrow \infty} G(u)=-\infty$. Also $G$ has a single strictly positive maximum on $[0, \infty)$, that is there exists a single $u_{\max }>0$ such that

$$
G_{\max }:=G\left(u_{\max }\right)=\sup _{u \geq 0} G(u)>0
$$

and the inverse image of the set $\left[0, G_{\max } / 2\right]$ under $G$ is given by

$$
G^{-1}\left(\left[0, G_{\max } / 2\right)=\left[0, u_{1}\right] \cup\left[u_{2}, \infty\right)\right.
$$

for some $0<u_{1}<u_{\max }<u_{2}<\infty$.

Note that $\lim _{\tau \rightarrow \infty} R_{2}(\tau)=0$ since $\beta$ and $R_{1}$ are going to zero at infinity and $\lim _{\tau \rightarrow \infty} \tilde{\tau}=$ $\infty$. Choose $\tau_{2} \geq \tau_{1}$ so large that $R_{2}(\tau) \leq G_{\max } / 2$ for all $\tau \geq \tau_{2}$. Then (6.11) shows that

$$
\|\beta\|_{\nu, \varepsilon, \tau} \in\left[0, u_{1}\right] \cup\left[u_{2}, \infty\right)
$$

for all $\tau \geq \tau_{2}$ and all $0<\varepsilon \leq 1$, as long as $\nu=\frac{1}{\tau \ln \tau}$.

Step 1: Because of Lemma (iv), for fixed $\nu, \tau>0$, the map $0<\varepsilon \mapsto\|\beta\|_{\nu, \varepsilon, \tau}$ is continuous. So since $u_{1}<u_{2}$, the intermediate value theorem for continuous functions and (6.12) show that we have, for all $\tau \geq \tau_{2}$ and $\nu=\frac{1}{\tau \ln \tau}$, the dichotomy

either $0 \leq\|\beta\|_{\nu, \varepsilon, \tau} \leq u_{1}$ for all $0<\varepsilon \leq 1$, or $u_{2} \leq\|\beta\|_{\nu, \varepsilon, \tau}$ for all $0<\varepsilon \leq 1$.

Step 2: Since $H_{\tau, 1} \leq 1$ by Lemma (i), we have $\|\beta\|_{\nu, 1, \tau}=\beta(\tau) \rightarrow 0$ as $\tau \rightarrow \infty$. So we can choose $\tau \geq \tau_{2}$ so large such that $\|\beta\|_{\nu, 1, \tau} \leq u_{1}$. For this $\tau$ we have from Step 1 that

$$
\|\beta\|_{\nu, \varepsilon, \tau} \leq u_{1} \text { for all } 0<\varepsilon \leq 1
$$

where $\nu=\frac{1}{\tau \ln \tau}>0$. Thus also

$$
\|\beta\|_{\nu, 0, \tau}=\lim _{\varepsilon \rightarrow 0}\|\beta\|_{\nu, \varepsilon, \tau} \leq u_{1}<\infty
$$

by Lemma (v). This finishes the proof of Proposition 6.2.

Now we come to the 
Proof of Lemma 6.4. Part (i) is clear from the definition of $H_{\nu, \varepsilon}$. For part (ii) we note that for fixed $0<\sigma<1$ and $m:=\lfloor\sigma(l+1)\rfloor$, one has $m \leq \sigma(l+1)<m+1$, hence

$$
\begin{aligned}
H_{\nu}(l)(m+1)^{-(m+1) / 4} & \leq(l+1)^{\nu(l+1)}(\sigma(l+1))^{-\sigma(l+1) / 4} \\
& =\exp \left(-\left(\left(\frac{\sigma}{4}-\nu\right) \ln (l+1)+\frac{\sigma \ln \sigma}{4}\right)(l+1)\right) \\
& \leq \exp \left(-\left(\frac{\sigma}{8} \ln (l+1)-\frac{1}{4 e}\right)(l+1)\right)
\end{aligned}
$$

since $0<-\sigma \ln \sigma \leq e^{-1}$ for all $0<\sigma<1$ and $\nu \leq \sigma / 8$. This proves (6.5).

Of course, (6.6) holds with constant

$$
C:=\sup _{l \in \mathbb{N}_{0}} \sup _{0 \leq \varepsilon \leq 1} g(n, l, \varepsilon)
$$

where $n=l-\lfloor\sigma(l+1)\rfloor$ and

$$
g(n, l, \varepsilon):=\frac{H_{\nu, \varepsilon}(l)}{H_{\nu, \varepsilon}(n)^{\theta}}=\frac{\left(H_{\nu}(n)^{-1}+\varepsilon\right)^{\theta}}{H_{\nu}(l)^{-1}+\varepsilon}
$$

where we droped, for simplicity of notation, the dependence of $g$ on $\theta$ and $\nu$. Since $H_{\nu, \varepsilon} \geq 1$, $g$ is certainly decreasing in $\theta>1$, and so is $C$. A simple computation shows

$$
\frac{\partial}{\partial \varepsilon} g(n, l, \varepsilon)=\frac{\left(H_{\nu}(n)^{-1}+\varepsilon\right)^{\theta-1}}{\left(H_{\nu}(l)^{-1}+\varepsilon\right)^{2}}\left(\theta H_{\nu}(l)^{-1}-H_{\nu}(n)^{-1}+(\theta-1) \varepsilon\right) .
$$

Since $n \leq l$ and $\theta>1$, the map $0 \leq \varepsilon \mapsto \theta H_{\nu}(l)^{-1}-H_{\nu}(n)^{-1}+(\theta-1) \varepsilon$ is either positive for all $\varepsilon \geq 0$, or it is negative for small and positive for large $\varepsilon$, with a single zero for some $\varepsilon>0$. Thus the map $0 \leq \varepsilon \mapsto g(n, l, \varepsilon)$ is either increasing in $\varepsilon \geq 0$, or it decreasing for small and increasing for large $\varepsilon \geq 0$, with a single minimum at some $\varepsilon>0$ and no maximum in $(0, \infty)$. Thus the supremum of $g(n, l, \varepsilon)$ over $0 \leq \varepsilon \leq 1$ is attained at the boundary,

$$
\sup g(n, l, \varepsilon)=\max (g(n, l, 0), g(n, l, 1))
$$

for all $0 \leq n \leq l$. We have

$$
g(n, l, 1)=\frac{\left(H_{\nu}(n)^{-1}+1\right)^{\theta}}{H_{\nu}(l)^{-1}+1} \leq 2^{\theta}
$$

for all $n, l \in \mathbb{N}_{0}$ and, because $n=l-\lfloor\sigma(l+1)\rfloor \geq l-\sigma(l+1)=(1-\sigma)(l+1)-1$,

$$
\begin{aligned}
g(n, l, 0) & \leq \frac{(l+1)^{\nu(l+1)}}{((1-\sigma)(l+1))^{\theta \nu(1-\sigma)(l+1)}} \\
& =\exp (\nu[(1-\theta(1-\sigma))(l+1) \ln (l+1)-\theta(1-\sigma) \ln (1-\sigma)(l+1)]) \\
& \leq \exp \left(-\nu\left[(\theta(1-\sigma)-1) \ln (l+1)-e^{-1} \theta\right](l+1)\right)
\end{aligned}
$$

A short calculation reveals that for $a, b>0$ the maximum of $B(s)=-(a \ln s-b) s$ over $s>0$ is attained at $B_{\max }=a e^{\frac{b}{a}-1}$ so with $a=\theta(1-\sigma)-1$ and $b=e^{-1} \theta$ this shows

$$
g(n, l, 0) \leq \exp \left(\nu(\theta(1-\sigma)-1) \exp \left(\frac{e^{-1} \theta}{(\theta(1-\sigma)-1)}-1\right)\right)
$$

for all $l \in \mathbb{N}_{0}$ and with $n=l-\lfloor\sigma(l+1)\rfloor$ as long as $\theta(1-\sigma)>1$, which in turn is equivalent to $\sigma<\frac{\theta-1}{\theta}$. This proves (6.6) and alos shows that the constant $C$ is increasing in $\nu$.

To prove part (iv) note that because for the triangle inequality

$$
\left|\|\beta\|_{\nu^{\prime}, \varepsilon^{\prime}, \tau}-\|\beta\|_{\nu, \varepsilon, \tau}\right| \leq \sup _{l \in \mathbb{N}_{0}}\left|H_{\nu^{\prime}, \varepsilon^{\prime}}(l)-H_{\nu, \varepsilon}(l)\right| \sup _{l \in \mathbb{N}_{0}}|\beta(l)|
$$


for all $\nu, \nu^{\prime} \geq 0$ and $\varepsilon, \varepsilon^{\prime}>0$. Note that

$$
\sup _{l \in \mathbb{N}_{0}}\left|H_{\nu^{\prime}, \varepsilon^{\prime}}(l)-H_{\nu, \varepsilon}(l)\right| \leq \sup _{s \in[0,1]}\left|h\left(\nu^{\prime}, \varepsilon^{\prime}, s\right)-h(\nu, \varepsilon, s)\right|
$$

with $h(\nu, \varepsilon, s):=\left(s^{\nu s}+\varepsilon\right)^{-1}$. The function $h$ is continuous on $[0, \infty) \times(0, \infty) \times[0,1]$ and thus uniformly continuous on $\left[0, \kappa^{-1}\right] \times\left[\kappa^{-1}, \kappa\right] \times[0,1]$ for any $\kappa>0$. Thus, for any $r>0$ there exist $\delta>0$ with $\left|h\left(\nu^{\prime}, \varepsilon^{\prime}, s^{\prime}\right)-h(\nu, \varepsilon, s)\right| \leq r$ as long as $0 \leq \nu, \nu^{\prime} \leq \kappa, \kappa^{-1} \leq \varepsilon, \varepsilon^{\prime} \leq \kappa$ and $0 \leq s, s^{\prime} \leq 1$ are such that $\left|\nu^{\prime}-\nu\right|,\left|\varepsilon^{\prime}-\varepsilon\right|,\left|s^{\prime}-s\right| \leq \delta$. Thus for these $\nu, \nu^{\prime}$ and $\varepsilon, \varepsilon^{\prime}$ also

$$
\sup _{0 \leq s \leq 1}\left|h\left(\nu^{\prime}, \varepsilon^{\prime}, s\right)-h(\nu, \varepsilon, s)\right| \leq r
$$

Hence also

$$
\sup _{l \in \mathbb{N}_{0}}\left|H_{\nu^{\prime}, \varepsilon^{\prime}}(l)-H_{\nu, \varepsilon}(l)\right| \leq \sup _{s \in[0,1]}\left|h\left(\nu^{\prime}, \varepsilon^{\prime}, s\right)-h(\nu, \varepsilon, s)\right| \leq r
$$

for all $0 \leq \nu, \nu^{\prime} \leq \kappa, \kappa^{-1} \leq \varepsilon, \varepsilon^{\prime} \leq \kappa$ with $\left|\nu^{\prime}-\nu\right|,\left|\varepsilon^{\prime}-\varepsilon\right| \leq \delta$. Since $\kappa>1$ is arbitrary, this shows the continuity of $\|\beta\|_{\nu, \varepsilon, \tau}$ in $\nu \geq 0$ and $\varepsilon>0$.

To prove the last claim, we simply note that $H_{\nu, \varepsilon}$ is decreasing in $\varepsilon>0$, so the map $0<\varepsilon \mapsto\|\beta\|_{\nu, \varepsilon, \tau}$ is decreasing. By the monotone convergence theorem and since one can interchange suprema, we get

$$
\begin{aligned}
\lim _{\varepsilon \rightarrow 0}\|\beta\|_{\nu, \varepsilon, \tau} & =\sup _{0<\varepsilon \leq 1}\|\beta\|_{\nu, \varepsilon, \tau}=\sup _{0<\varepsilon \leq 1} \sup _{l \geq \tau} H_{\nu, \varepsilon}(l) \beta(l) \\
& =\sup _{l \geq \tau} \sup _{0<\varepsilon \leq 1} H_{\nu, \varepsilon}(l) \beta(l)=\sup _{l \geq \tau} H_{\nu, 0}(l) \beta(l)=\|\beta\|_{\nu, 0, \tau}
\end{aligned}
$$

which proves (6.7) and finishes the proof of Lemma 6.4.

\subsection{Boosting the (super-exponential) decay rate.}

Proposition 6.5 (Boosting the super-exponential decay rate). Let $\beta$ be a non-negative function which obeys the self-consistency bound (6.2) of Proposition 6.1 for some $\theta>1$ and some $0<\alpha<\frac{1}{2}$. Furthermore, assume that for some $\nu>0$ we have

$$
\beta(l) \lesssim(l+1)^{-\nu(l+1)} \quad \text { for all } l \geq 0 .
$$

Then for all $0<\alpha_{1}<\alpha$, setting $\nu_{1}:=\frac{2 \theta \alpha_{1}}{\alpha_{1}+\theta \nu} \nu$, we have

$$
\beta(l) \lesssim(l+1)^{-\nu_{1}(l+1)} \quad \text { for all } l \geq 0 .
$$

Remark 6.6. $\nu_{1}>\nu$ is equaivalent to $\nu<\frac{2 \theta-1}{\theta} \alpha_{1}$. So Proposition 6.5 allows us to boost the decay rate as long as $\nu<\frac{2 \theta-1}{\theta} \alpha$, since $\nu<\frac{2 \theta-1}{\theta} \alpha_{1}$ whenever $\alpha_{1}$ close enough to $\alpha$.

Proof. The self-consistency bound (6.2) and our assumptions on $\beta$ imply

$$
\beta(l) \lesssim(n+1)^{-\theta \nu(n+1)}+(m+1)^{-\alpha(m+1)}
$$

for all $l, m, n \in \mathbb{N}_{0}$ with $l=n+m$.

Set $m=\lfloor\sigma(l+1)\rfloor$ for some $0<\sigma<1$, which we choose later. Then $m \leq \sigma(l+1)<$ $\sigma(l+1)+1$ and for $n=l-m$ we have $(1-\sigma)(l+1)-1 \leq n<(1-\sigma)(l+1)$, that is, $n=\lfloor(1-\sigma)(l+1)-1\rfloor$. Then the self-consistency bound implies

$$
\begin{aligned}
\beta(l) & \lesssim((1-\sigma)(l+1))^{-\theta \nu(1-\sigma)(l+1)}+(\sigma(l+1))^{-\alpha \sigma(l+1)} \\
& =\exp (-(\theta \nu(1-\sigma) \ln (1-\sigma)+\alpha \sigma \ln \sigma)(l+1)-(\theta \nu(1-\sigma)+\alpha \sigma)(l+1) \ln (l+1)) \\
& \leq \exp \left((\theta \nu+\alpha) e^{-1}(l+1)-(\theta \nu(1-\sigma)+\alpha \sigma)(l+1) \ln (l+1)\right)
\end{aligned}
$$




$$
\lesssim \exp \left(-\left(\theta \nu(1-\sigma)+\alpha_{1} \sigma\right)(l+1) \ln (l+1)\right)
$$

for any $0<\alpha_{1}<\alpha$ and all $l \in \mathbb{N}_{0}$, where we also used $\sigma \ln \sigma \geq-e^{-1}$ and $(1-\sigma) \ln (1-\sigma) \geq$ $-e^{-1}$ for all $0<\sigma<1$ in the third line.

We choose $\sigma$ such that $\theta \nu(1-\sigma)=\alpha_{1} \sigma$, equivalently

$$
\sigma=\frac{\theta \nu}{\alpha_{1}+\theta \nu} .
$$

This yields $0<\sigma<1$ and $\theta \nu(1-\sigma)+\alpha_{1} \sigma=2 \alpha_{1} \sigma=\frac{2 \theta \alpha_{1}}{\alpha_{1}+\theta \nu} \nu=\nu_{1}$. So

$$
\beta(l) \lesssim \exp \left(-\nu_{1}(l+1) \ln (l+1)\right)
$$

for all $l \in \mathbb{N}_{0}$, which finished the proof.

Corollary 6.7. Let $\beta: \mathbb{N}_{0} \rightarrow \mathbb{R}$ be a decreasing non-negative function, vanishing at infinity, which obeys the self-consistency bound (6.2) of Proposition 6.1 for some $\theta>1$ and all $0<\alpha<1$. Furthermore, recall $H_{\nu}(l)=(l+1)^{\nu(l+1)}$ for $l \in \mathbb{N}_{0}$ and $\nu \in \mathbb{R}$. Then

$$
\nu_{* *}=\sup \left\{\nu>0 \mid \beta \lesssim H_{-\nu}\right\} \geq 1-\frac{1}{2 \theta}
$$

Proof. From Proposition 6.2 we know that $\nu_{* *}>0$. Let $0<\nu<\nu_{* *}$ and $0<\alpha_{1}<\frac{1}{2}$, then Proposition 6.5 shows

$$
\beta \lesssim H_{-\nu_{1}}
$$

for $\nu_{1}:=\frac{2 \theta \alpha_{1} \nu}{\alpha_{1}+\theta \nu}$. Thus, by the definition of $\nu_{* *}$ we have

$$
\frac{2 \theta \alpha_{1} \nu}{\alpha_{1}+\theta \nu} \leq \nu_{* *}
$$

for all $0<\nu<\nu_{* *}$ and all $0<\alpha_{1}<\frac{1}{2}$. Taking first the limit $\nu \nearrow \nu_{* *}$ and then $\alpha_{1} \nearrow \frac{1}{2}$ in the above inequality shows

$$
\frac{\theta \nu_{* *}}{\frac{1}{2}+\theta \nu_{* *}} \leq \nu_{* *} .
$$

Since $\nu_{* *}>0$ this implies $\nu_{* *} \geq 1-\frac{1}{2 \theta}$.

Now we can give the

Proof of Theorem 1.7. Let $\theta=\gamma_{1}-1$ and $\varphi$ be a solution of (1.2). Then Propsition 6.1 shows that the tail distribution $\beta$ of $\varphi$ obeys the self-conistency bound (6.2). Then the claim follows from $|\varphi(x)| \leq \beta(|x|)$ for all $x \in \mathbb{Z}$ and Corollary 6.7.

\section{Appendix A. Some useful bounds}

We start with

Lemma A.1. (i) Let $1 \leq p<\infty$ and $f_{1}, f_{2} \in l^{p}(\mathbb{Z})$, then

$$
\left|\left\|f_{1}\right\|_{p}^{p}-\left\|f_{2}\right\|_{p}^{p}\right| \leq p \max \left(\left\|f_{1}\right\|_{p}^{p-1},\left\|f_{2}\right\|_{p}^{p-1}\right)\left\|f_{1}-f_{2}\right\|_{p}
$$

(ii) The free time evolution group $T_{r}=e^{i r \Delta}$ is bounded on $l^{p}(\mathbb{Z})$ for all $1 \leq p \leq \infty$ with

$$
\left\|T_{r} f\right\|_{p} \leq e^{4\left|r \| 1-\frac{2}{p}\right|}\|f\|_{p}
$$

(iii) The group $T_{r}=e^{i r \Delta}$ is norm continuous on $l^{p}$ for any $1 \leq p \leq \infty$ with

$$
\left\|f-T_{r} f\right\|_{p} \leq\left(e^{4|r|}-1\right)\|f\|_{p}
$$


(iv) For the kernel ${ }^{5}$ of $T_{r}$, one has the bound

$$
\left|\left\langle x\left|T_{r}\right| y\right\rangle\right| \leq \min \left(1, e^{4|r|} \frac{(4|r|)^{|x-y|}}{|x-y| !}\right) .
$$

(v) (Strong bilinear bound) For any $1 \leq p \leq \infty$

$$
\sup _{|r| \leq B}\left\|T_{r} f_{1} T_{r} f_{2}\right\|_{p} \leq \min \left(1, \frac{8 e^{16 B}(4 B)^{\left\lceil\frac{s}{2}\right\rceil}}{\left\lceil\frac{s}{2}\right\rceil !}\right)\left\|f_{1}\right\|_{2}\left\|f_{2}\right\|_{2}
$$

with $s:=\operatorname{dist}\left(\operatorname{supp} f_{1}, \operatorname{supp} f_{2}\right)$.

(vi) (Twisted strong bilinear bound) For any $1 \leq p \leq \infty$ and $B>0$

$$
\sup _{r \in[-B, B]}\left\|T_{r}\left(e^{F_{\nu, \varepsilon}} h_{1}\right) T_{r}\left(e^{-F_{\nu \varepsilon}} h_{2}\right)\right\|_{p} \leq 4 e^{8 B\left(1+e^{\nu}\right)} \min \left(1, \frac{\left.2\left(4 R e^{\nu}\right)^{\left\lceil\frac{s}{2}\right.}\right\rceil}{\left\lceil\frac{s}{2}\right\rceil !}\right)\left\|h_{1}\right\|_{2}\left\|h_{2}\right\|_{2}
$$

uniformly in $\varepsilon>0$. Here $s:=\operatorname{dist}\left(\operatorname{supp} h_{1}, \operatorname{supp} h_{2}\right) \geq 0$ and $F_{\nu, \varepsilon}(x)=\frac{\nu|x|}{1+\varepsilon|x|}$.

(vii) (Exchange of an exponential weight) For any $\alpha \geq 1,1 \leq p \leq \infty$, and $B>0$

$$
\sup _{|r| \leq B}\left\|T_{r}\left(e^{F_{\nu, \varepsilon}} h_{1}\right)\left|T_{r} h_{2}\right|^{\alpha}\right\|_{p} \leq\left(2 e^{4 B\left(1+e^{\nu}\right)}\right)^{\alpha+1}\left\|h_{1}\right\|_{2}\left\|e^{F_{\nu / \alpha, \varepsilon}} h_{2}\right\|_{2}^{\alpha} .
$$

(viii) For any $\nu>0$ and $A>0$, let $f_{\nu}(x):=A e^{-\nu|x|}$. Then

$$
\begin{gathered}
\left\|f_{\nu}\right\|_{\kappa}^{\kappa}=A^{\kappa} \frac{\cosh \left(\frac{\kappa}{2} \nu\right)}{\sinh \left(\frac{\kappa}{2} \nu\right)} \\
\left\langle f_{\nu},-\Delta f_{\nu}\right\rangle=\left\|D_{+} f_{\nu}\right\|_{2}^{2}=4 A^{2} \frac{\sinh ^{2}(\nu / 2)}{\sinh (\nu)} .
\end{gathered}
$$

Remark A.2. The strong bilinear bound above strengthens the strong bilinear bound from $[15,16]$, which was proven there only for $p=2$. Moreover, we will give a proof which is considerably simpler than the one in $[15,16]$. The twisted strong bilinear bound (A.6) is new and needed in the proof that solutions of (1.1) with $\omega<0$ have some exponential decay for positive average dispersion. It is important that the right hand side of (A.6) is independent of $\varepsilon>0$. The exchange of exponential weights bound (A.7) is crucial for our strategy of boosting the exponential decay rate to the one given by the physical heuristic. The main feature of (A.7) is that for $\alpha>1$ its right hand side has an exponential growth of order $\nu / \alpha$ which is strictly smaller than $\nu$ when $\nu>0$. Thus (A.7) allows us to absorb some excess exponential factor in the boosting argument of Section 5.2.

Proof. For the first claim, let $f_{1}, f_{2} \in l^{p}(\mathbb{Z}), 1 \leq p<\infty$ and note that for $a, b \geq 0$ one has

$$
\left|a^{p}-b^{p}\right| \leq p \max \left(a^{p-1}, b^{p-1}\right)|a-b|
$$

since, if $a \leq b$, then

$$
\left|a^{p}-b^{p}\right|=b^{p}-a^{p}=p \int_{a}^{b} s^{p-1} d s \leq p b^{p-1}(b-a)
$$

and the case $a \geq b$ follows by symmetry. Using $a=\left\|f_{1}\right\|_{p}$ and $b=\left\|f_{2}\right\|_{p}$ in (A.9) shows

$$
\left|\left\|f_{1}\right\|_{p}^{p}-\left\|f_{2}\right\|_{p}^{p}\right| \leq p \max \left(\left\|f_{1}\right\|_{p}^{p-1},\left\|f_{2}\right\|_{p}^{p-1}\right)\left|\left\|f_{1}\right\|_{p}-\left\|f_{2}\right\|_{p}\right|
$$

which gives (A.1).

\footnotetext{
${ }^{5}$ We use the physicists' notation $\left\langle x\left|T_{r}\right| y\right\rangle$ for the kernel, for mathematicians, $\left\langle x\left|T_{r}\right| y\right\rangle=\left\langle\delta_{x}, T_{r} \delta_{y}\right\rangle$, where $\delta_{x}$ is the Kronecker delta at $x \in \mathbb{Z}$.
} 
As a preparation for the proof of the other claims, note that $T_{r}$ has the norm continuous series expansion

$$
T_{r}=\sum_{n=0}^{\infty} \frac{(i r)^{n}}{n !} \Delta^{n}
$$

One easily sees that

$$
\|\Delta f\|_{1} \leq 4\|f\|_{1} \text { and }\|\Delta f\|_{\infty} \leq 4\|f\|_{\infty} .
$$

Thus the norm of $\Delta$ on $l^{\infty}(\mathbb{Z})$ and $l^{1}(\mathbb{Z})$ is bounded by 4 and from the power series for $T_{r}$ one sees

$$
\left\|T_{r} f\right\|_{p} \leq \sum_{n=0}^{\infty} \frac{(4|r|)^{n}}{n !}\|f\|_{p}=e^{4|r|}\|f\|_{p}, p=1 \text { or } \infty .
$$

By self-adjointness of $\Delta$ on $l^{2}(\mathbb{Z})$ one has that $T_{r}$ is unitary on $l^{2}(\mathbb{Z})$, so $\left\|T_{r} f\right\|_{2}=\|f\|_{2}$ and interpolating this with the bound on $l^{1}(\mathbb{Z})$ and $l^{\infty}(\mathbb{Z})$ with the help of the Riesz-Thorin interpolation theorem proves (A.2).

Moreover, applying Riesz-Thorin interpolation theorem on (A.10) yields $\|\Delta f\|_{p} \leq 4\|f\|_{p}$ for all $1 \leq p \leq \infty$. The series expansion for $T_{r}$ then yields

$$
\left\|f-T_{r} f\right\|_{p} \leq \sum_{n=1}^{\infty} \frac{(4|r|)^{n}}{n !}\|f\|_{p}=\left(e^{4|r|}-1\right)\|f\|_{p}
$$

which is (A.3). In particular, $T_{r}$ is norm continuous on $l^{p}(\mathbb{Z})$ at $r=0$, which together with the group property of $T_{r}$ and (A.2) shows its continuity for all $r$.

Because of the norm convergent series expansion for $T_{r}$, its 'kernel' $\left\langle x\left|T_{r}\right| y\right\rangle$, for which one has $T_{r} f(x)=\sum_{y \in \mathbb{Z}}\left\langle x\left|T_{r}\right| y\right\rangle f(y)$, is given by

$$
\left\langle x\left|T_{r}\right| y\right\rangle=\sum_{n=0}^{\infty} \frac{(i r)^{n}}{n !}\left\langle x\left|\Delta^{n}\right| y\right\rangle=\sum_{n=|x-y|}^{\infty} \frac{(i r)^{n}}{n !}\left\langle x\left|\Delta^{n}\right| y\right\rangle
$$

since $\left\langle x\left|\Delta^{n}\right| y\right\rangle=0$ if $n<|x-y|$. Moreover, $\left|\left\langle x\left|\Delta^{n}\right| y\right\rangle\right| \leq\|\Delta\|^{n}=4^{n}$, so we have the bound

$$
\left|\left\langle x\left|T_{r}\right| y\right\rangle\right| \leq \sum_{n=|x-y|}^{\infty} \frac{(4|r|)^{n}}{n !} .
$$

By unicity of $T_{r}$ and the Cauchy-Schwartz inequality one always has $\left|\left\langle x\left|T_{r}\right| y\right\rangle\right| \leq 1$. Moreover, by (A.12), we have

$$
\begin{aligned}
\left|\left\langle x\left|T_{r}\right| y\right\rangle\right| & \leq \sum_{n=|x-y|}^{\infty} \frac{(4|r|)^{n}}{n !}=(4|r|)^{|x-y|} \sum_{n=0}^{\infty} \frac{(4|r|)^{n}}{(|x-y|+n) !} \\
& \leq \frac{(4|r|)^{|x-y|}}{|x-y| !} \sum_{n=0}^{\infty} \frac{(4|r|)^{n}}{n !}=\frac{e^{4|r|}(4|r|)^{|x-y|}}{|x-y| !}
\end{aligned}
$$

since $(|x-y|+n) ! \geq|x-y|$ !n!. So (A.4) follows.

To prove the fifth claim, we first note that on the sequence spaces $l^{p}(\mathbb{Z})$, the bound $\|h\|_{p} \leq\|h\|_{1}$ holds. Hence $\left\|T_{r} f_{1} T_{r} f_{2}\right\|_{p} \leq\left\|T_{r} f_{1} T_{r} f_{2}\right\|_{1}$, so we only have to prove (A.5) for $p=1$. Because of the Cauchy Schwarz inequality,

$$
\left\|T_{r} f_{1} T_{r} f_{2}\right\|_{1} \leq\left\|T_{r} f_{1}\right\|_{2}\left\|T_{r} f_{2}\right\|_{2}=\left\|f_{1}\right\|_{2}\left\|f_{2}\right\|_{2}
$$


Now let $s:=\operatorname{dist}\left(\operatorname{supp} f_{1}, \operatorname{supp} f_{2}\right)>0$. Then with

$$
A_{r}\left(y_{1}, y_{2}\right):=e^{8|r|} \sum_{x} \frac{(4|r|)^{\left|x-y_{1}\right|}}{\left|x-y_{1}\right| !} \frac{(4|r|)^{\left|x-y_{2}\right|}}{\left|x-y_{2}\right| !}
$$

the bound (A.4), the Cauchy-Schwarz inequality and the symmetry of $A_{r}$ in $y_{1}$ and $y_{2}$ gives

$$
\begin{aligned}
\left\|T_{r} f_{1} T_{r} f_{2}\right\|_{1} & \leq \sum_{y_{1}, y_{2}}\left|f\left(y_{1}\right)\right| A_{r}\left(y_{1}, y_{2}\right)\left|f\left(y_{2}\right)\right| \\
& \leq\left(\sum_{\substack{y_{1} \in \mathbb{Z} \\
y_{2} \in \operatorname{supp} f_{2}}}\left|f\left(y_{1}\right)\right|^{2} A_{r}\left(y_{1}, y_{2}\right)\right)^{1 / 2}\left(\sum_{\substack{y_{2} \in \mathbb{Z} \\
y_{1} \in \operatorname{supp} f_{1}}} A_{r}\left(y_{1}, y_{2}\right)\left|f\left(y_{2}\right)\right|^{2}\right)^{1 / 2} \\
& \leq\left(A_{r, 1,2} A_{r, 2,1}\right)^{1 / 2}\left\|f_{1}\right\|_{2}\left\|f_{2}\right\|_{2}
\end{aligned}
$$

where $A_{r, l, m}=\sup _{y_{1} \in \operatorname{supp} f_{l}} \sum_{y_{2} \in \operatorname{supp} f_{m}} A_{r}\left(y_{1}, y_{2}\right)$.

Fix $y_{1} \in \operatorname{supp} f_{1}$, then for all $x \in \mathbb{Z}$ and all $y_{2} \in \operatorname{supp} f_{2}$ we have $\left|x-y_{1}\right| \geq \frac{s}{2}$ or $\left|x-y_{2}\right| \geq \frac{s}{2}$ and since the distance is always an integer, setting $\lceil s\rceil:=\min \{n \in \mathbb{Z} \mid s \leq n\}$ and denoting $G_{r}(y):=\frac{(4|r|)^{|y|}}{|y| !}$, we get

$$
\begin{aligned}
& \sum_{y_{2} \in \operatorname{supp} f_{2}} A_{r}\left(y_{1}, y_{2}\right)=e^{8|r|} \sum_{y_{2} \in \operatorname{supp} f_{2}} \sum_{x \in \mathbb{Z}} G_{r}\left(x-y_{1}\right) G_{r}\left(x-y_{2}\right) \\
\leq & e^{8|r|}\left(\sum_{\substack{y_{2}, x \\
\left|x-y_{1}\right| \geq\left\lceil\frac{s}{2}\right\rceil}} G_{r}\left(x-y_{1}\right) G_{r}\left(x-y_{2}\right)+\sum_{\substack{y_{2}, x \\
\left|x-y_{2}\right| \geq\left\lceil\frac{s}{2}\right\rceil}} G_{r}\left(x-y_{1}\right) G_{r}\left(x-y_{2}\right)\right) \\
= & e^{8|r|}\left(\sum_{|x| \geq\left\lceil\frac{s}{2}\right\rceil} G_{r}\left(y_{1}\right) \sum_{y_{2}} G_{r}\left(y_{2}\right)+\sum_{x} G_{r}(x) \sum_{\left|y_{2}\right| \geq\left\lceil\frac{s}{2}\right\rceil} G_{r}\left(y_{2}\right)\right) \\
= & 2 e^{8|r|} \sum_{|x| \geq\left\lceil\frac{s}{2}\right\rceil} G_{r}(x) \sum_{y} G_{r}(y) .
\end{aligned}
$$

A simple calculation gives

$$
\sum_{y} G_{r}(y)=G_{r}(0)+2 \sum_{y \geq 1} G_{r}(y)=1+2 \sum_{y=1}^{\infty} \frac{(4|r|)^{y}}{y !} \leq 2 e^{4|r|}
$$

and

$$
\sum_{|x| \geq\left\lceil\frac{s}{2}\right\rceil} G_{r}(x)=2 \sum_{x=\left\lceil\frac{s}{2}\right\rceil}^{\infty} \frac{(4|r|)^{x}}{x !}=2(4|r|)^{\left\lceil\frac{s}{2}\right\rceil} \sum_{n=0}^{\infty} \frac{(4|r|)^{n}}{\left(\left\lceil\frac{s}{2}\right\rceil+n\right) !} \leq \frac{2 e^{4|r|}(4|r|)^{\left\lceil\frac{s}{2}\right\rceil}}{\left\lceil\frac{s}{2}\right\rceil !} .
$$

Thus

$$
A_{r, 1,2} \leq \frac{8 e^{16|r|}(4|r|)^{\left\lceil\frac{s}{2}\right\rceil}}{\left\lceil\frac{s}{2}\right\rceil !}
$$

The same argument yields the same bound for $A_{2,1}$ and since

$$
\left.\left\|T_{r} f_{1} T_{r} f_{2}\right\|_{1} \leq \min \left(1,\left(A_{r, 1,2} A_{r, 2,1}\right)\right)^{1 / 2}\right)\left\|f_{1}\right\|_{2}\left\|f_{2}\right\|_{2}
$$

this proves (A.5).

In order to prove (A.6), we can again, without loss of generality, consider the case $p=1$. Fix $\nu \geq 0$ and $\varepsilon>0$ and let $F=F_{\nu, \varepsilon}$. Noting

$$
\left\|T_{r}\left(e^{F} h_{1}\right) T_{r}\left(e^{-F} h_{2}\right)\right\|_{1}=\left\|e^{-F} T_{r}\left(e^{F} h_{1}\right) e^{F} T_{r}\left(e^{-F} h_{2}\right)\right\|_{1}
$$




$$
\leq \sum_{x \in \mathbb{Z}} \sum_{y_{1}, y_{2} \in \mathbb{Z}} e^{\left|F(x)-F\left(y_{1}\right)\right|}\left|\left\langle x\left|T_{r}\right| y_{1}\right\rangle\right|\left|h_{1}\left(y_{1}\right)\right| e^{\left|F(x)-F\left(y_{2}\right)\right|}\left|\left\langle x\left|T_{r}\right| y_{2}\right\rangle\right|\left|h_{2}\left(y_{2}\right)\right|
$$

and, because of the reverse triangle inequality for $F$, we have $|F(x)-F(y)| \leq F(x-y) \leq$ $\nu|x-y|$. Thus setting $G_{r, \nu}(y):=\frac{\left(4|r| e^{\nu}\right)^{|y|}}{|y| !}$ the bound (A.4) yields

$$
\left\|T_{r}\left(e^{F} h_{1}\right) T_{r}\left(e^{-F} h_{2}\right)\right\|_{1} \leq e^{8|r|} \sum_{y_{1}, y_{2} \in \mathbb{Z}}\left|h_{1}\left(y_{1}\right)\right|\left(\sum_{x \in \mathbb{Z}} G_{r, \nu}\left(x-y_{1}\right) G_{r, \nu}\left(x-y_{2}\right)\right)\left|h_{2}\left(y_{2}\right)\right|
$$

so denoting $A_{r, \nu}\left(y_{1}, y_{2}\right):=\sum_{x \in \mathbb{Z}} G_{r, \nu}\left(x-y_{1}\right) G_{r, \nu}\left(x-y_{2}\right)$, we can argue as for the bound (A.5), except now we cannot simply use unitarity of $T_{r}$ to get the bound when the supports of $h_{1}$ and $h_{2}$ are not separated. Instead, as in (A.14), we use and Cauchy-Schwartz and the symmetry of $A_{r, \nu}\left(y_{1}, y_{2}\right)$ in $y_{1}$ and $y_{2}$ to see

$$
\sum_{y_{1}, y_{2} \in \mathbb{Z}}\left|h_{1}\left(y_{1}\right)\right| A_{r, \nu}\left(y_{1}, y_{2}\right)\left|h_{2}\left(y_{2}\right)\right| \leq\left(\sup _{y_{1} \in \mathbb{Z}} \sum_{y_{2} \in \mathbb{Z}} A_{r, \nu}\left(y_{1}, y_{2}\right)\right)\left\|h_{1}\right\|_{2}\left\|h_{2}\right\|_{2}
$$

By translation invariance,

$$
\begin{aligned}
\sum_{y_{2} \in \mathbb{Z}} A_{r, \nu}\left(y_{1}, y_{2}\right) & =\sum_{x} \sum_{y_{2}} G_{r, \nu}\left(x-y_{1}\right) G_{r, \nu}\left(x-y_{2}\right)=\left(\sum_{y} G_{r, \nu}(y)\right)^{2} \\
& =\left(1+2 \sum_{n \in \mathbb{N}} \frac{\left(4|r| e^{\nu}\right)^{n}}{n !}\right)^{2} \leq 4 e^{8|r| e^{\nu}}
\end{aligned}
$$

Thus

$$
\left\|T_{r}\left(e^{F_{\nu, \varepsilon}} h_{1}\right) T_{r}\left(e^{-F_{\nu, \varepsilon}} h_{2}\right)\right\|_{1} \leq 4 e^{8|r|\left(1+e^{\nu}\right)}\left\|h_{1}\right\|_{2}\left\|h_{2}\right\|_{2}
$$

and proceeding similarly as in (A.14)-(A.18), one sees

$$
\left\|T_{r}\left(e^{F_{\nu, \varepsilon}} h_{1}\right) T_{r}\left(e^{-F_{\nu, \varepsilon}} h_{2}\right)\right\|_{1} \leq 8 e^{8|r|\left(1+e^{\nu}\right)} \frac{\left(4|r| e^{\nu}\right)^{\left\lceil\frac{s}{2}\right\rceil}}{\left\lceil\frac{s}{2}\right\rceil !}
$$

when $s:=\operatorname{dist}\left(\operatorname{supp} h_{1}, \operatorname{supp} h_{2}\right) \geq 1$. Together, (A.19) and (A.20) prove (A.6).

To prove (A.7), let $\alpha \geq 1$. Again, it is enough to consider the case $p=1$. Then with $\widetilde{h_{2}}:=e^{F_{\nu / \alpha, \varepsilon}} h_{2}$

$$
\begin{aligned}
\left\|T_{r}\left(e^{F_{\nu, \varepsilon}} h_{1}\right)\left|T_{r}\left(h_{2}\right)\right|^{\alpha}\right\|_{1} & =\left\|e^{-F_{\nu, \varepsilon}} T_{r}\left(e^{F_{\nu, \varepsilon}} h_{1}\right)\left|e^{F_{\nu / \alpha, \varepsilon}} T_{r}\left(e^{-F_{\nu / \alpha, \varepsilon} \widetilde{h_{2}}}\right)\right|^{\alpha}\right\|_{1} \\
& \leq\left\|e^{-F_{\nu, \varepsilon}} T_{r}\left(e^{F_{\nu, \varepsilon}} h_{1}\right) e^{F_{\nu / \alpha, \varepsilon}} T_{r}\left(e^{-F_{\nu / \alpha, \varepsilon} \widetilde{h_{2}}}\right)\right\|_{1}\left\|e^{F_{\nu / \alpha, \varepsilon}} T_{r}\left(e^{-F_{\nu / \alpha, \varepsilon} \widetilde{h_{2}}}\right)\right\|_{\infty}^{\alpha-1} .
\end{aligned}
$$

Now arguing similarly as in the proof of (A.19),

$$
\begin{aligned}
& \left\|e^{-F_{\nu, \varepsilon}} T_{r}\left(e^{F_{\nu, \varepsilon}} h_{1}\right) e^{F_{\nu / \alpha, \varepsilon}} T_{r}\left(e^{-F_{\nu / \alpha, \varepsilon}} \widetilde{h_{2}}\right)\right\|_{1} \\
& \quad \leq \sum_{x} \sum_{y_{1}, y_{2}} e^{\nu\left|x-y_{1}\right|}\left|\left\langle x\left|T_{r}\right| y_{1}\right\rangle\right|\left|h_{1}\left(y_{1}\right)\right| e^{(\nu / \alpha)\left|x-y_{2}\right|}\left|\left\langle x\left|T_{r}\right| y_{2}\right\rangle \| \widetilde{h_{2}}\left(y_{2}\right)\right| \\
& \leq 4 e^{4|r|\left(2+e^{\nu}+e^{\nu / \alpha}\right)}\left\|h_{1}\right\|_{2}\left\|\widetilde{h_{2}}\right\|_{2} \leq 4 e^{8|r|\left(1+e^{\nu}\right)}\left\|h_{1}\right\|_{2}\left\|\widetilde{h_{2}}\right\|_{2}
\end{aligned}
$$

and

$$
\begin{gathered}
\left\|e^{F_{\nu / \alpha, \varepsilon}} T_{r}\left(e^{-F_{\nu / \alpha, \varepsilon} \widetilde{h_{2}}}\right)\right\|_{\infty} \leq\left\|e^{F_{\nu / \alpha, \varepsilon}} T_{r}\left(e^{-F_{\nu / \alpha, \varepsilon} \widetilde{h_{2}}}\right)\right\|_{2}=\left\|e^{2 F_{\nu / \alpha, \varepsilon}}\left|T_{r}\left(e^{-F_{\nu / \alpha, \varepsilon} \widetilde{h_{2}}}\right)\right|^{2}\right\|_{1}^{1 / 2} \\
\leq\left(\sum_{x} \sum_{y_{1}, y_{2}} e^{(\nu / \alpha)\left|x-y_{1}\right|}\left|\left\langle x\left|T_{r}\right| y_{1}\right\rangle\right| \widetilde{h_{2}}\left(y_{1}\right)\left|e^{(\nu / \alpha)\left|x-y_{2}\right|}\right|\left\langle x\left|T_{r}\right| y_{2}\right\rangle\left|\widetilde{h_{2}}\left(y_{2}\right)\right|\right)^{1 / 2} \\
\leq 2 e^{4|r|\left(1+e^{\nu / \alpha}\right)}\left\|h_{1}\right\|_{2}\left\|\widetilde{h_{2}}\right\|_{2} \leq 2 e^{4|r|\left(1+e^{\nu}\right)}\left\|\widetilde{h_{2}}\right\|_{2}
\end{gathered}
$$


for all $\alpha \geq 1$. This proves (A.7).

Lastly, let $f_{\nu}(x)=A e^{-\nu|x|}$ with $\nu>0$ and $A>0$, then

$$
\left\|f_{\nu}\right\|_{\kappa}^{\kappa}=A^{\kappa} \sum_{x \in \mathbb{Z}} e^{-\kappa \nu|x|}=A^{\kappa}\left(1+2 \sum_{x=1}^{\infty} e^{-\kappa \nu x}\right)=A^{\kappa}\left(\frac{1+e^{-\kappa \nu x}}{1-e^{-\kappa \nu x}}\right)=A^{\kappa} \frac{\cosh \left(\frac{\kappa}{2} \nu\right)}{\sinh \left(\frac{\kappa}{2} \nu\right)} .
$$

Moreover,

$$
\begin{aligned}
\left\|D_{+} f_{\nu}\right\|_{2}^{2} & =A^{2} \sum_{x}\left|e^{-\nu|x+1|}-e^{-\nu|x|}\right|^{2}=A^{2}\left(\sum_{x \geq 0}\left(e^{-\nu}-1\right)^{2} e^{-2 \nu x}+\sum_{x \leq-1}\left(e^{\nu}-1\right)^{2} e^{2 \nu x}\right) \\
& =A^{2}\left(\left(e^{-\nu}-1\right)^{2} \frac{1}{1-e^{-2 \nu}}+\left(e^{\nu}-1\right)^{2} \frac{e^{-2 \nu}}{1-e^{-2 \nu}}\right)=4 A^{2} \frac{\sinh ^{2}(\nu / 2)}{\sinh (\nu)}
\end{aligned}
$$

\section{Appendix B. Boundedness, Negativity, And StRict Subadditivity of the} ENERGY

Recall that for $d_{\mathrm{av}} \geq 0$

$$
H(f):=\frac{d_{\mathrm{av}}}{2}\langle f,-\Delta f\rangle-N(f)
$$

and

$$
E_{\lambda}^{d_{\mathrm{av}}}:=\inf \left\{H(f):\|f\|^{2}=\lambda\right\} .
$$

In this section we will give an a-priori bound on the ground-state energy which is an essential ingredient in the construction of strongly convergent minimizing sequences.

Lemma B.1. Assume that assumption $\boldsymbol{A} 1$ holds. Then for every $\lambda \geq 0$

$$
-\lambda^{\gamma_{1} / 2}-\lambda^{\gamma_{2} / 2} \lesssim E_{\lambda}^{d_{\mathrm{av}}} \leq 0,
$$

where the implicit constant in the lower bound depends only on $\mu(\mathbb{R})$ and the support of $\mu$. In particular, the variational problem is well-posed.

Proof. The lower bound follows immediately from $H(f) \geq-N(f)$ and Proposition 2.6. For the upper bound we argue similarly as in the beginning of the proof of Theorem 3.1. Note that

$$
E_{\lambda}^{d_{\mathrm{av}}} \leq H(f)=\frac{d_{\mathrm{av}}}{2}\left\|D_{+} f\right\|_{2}^{2}-N(f) \leq \frac{d_{\mathrm{av}}}{2}\left\|D_{+} f\right\|_{2}^{2}+|N(f)|
$$

To bound the nonlinearity, we use (1.9) to see that with $B$ so that $\operatorname{supp} \mu \subset[-B, B]$,

$$
|N(f)| \lesssim \sup _{|r| \leq B}\left(\left\|T_{r} f\right\|_{\gamma_{1}}^{\gamma_{1}}+\left\|T_{r} f\right\|_{\gamma_{2}}^{\gamma_{2}}\right) \lesssim\|f\|_{\gamma_{1}}^{\gamma_{1}}+\|f\|_{\gamma_{2}}^{\gamma_{2}}
$$

where we also used the bound (A.2) from Lemma A.1. Now define $f_{n}(x)$ as in the proof of Theorem 3.1 by

$$
f_{n}(x):=c_{n} \mathbf{1}_{[-n, n]}(x)
$$

with $c_{n}=\left(\frac{\lambda}{2 n+1}\right)^{1 / 2}$. Then $\left\|f_{n}\right\|_{2}^{2}=\lambda$. Note that

$$
\left\|D_{+} f_{n}\right\|_{2}^{2}=2 c_{n}^{2} \rightarrow 0 \quad \text { as } n \rightarrow \infty
$$

and for any $\gamma>2$

$$
\left\|f_{n}\right\|_{\gamma}^{\gamma}=\left(\frac{\lambda}{2 n+1}\right)^{\gamma / 2}(2 n+1) \rightarrow 0
$$


as $n \rightarrow \infty$. So $E_{\lambda}^{d_{\text {av }}}=\inf _{\|f\|_{2}^{2}=\lambda} H(f) \leq \lim _{n \rightarrow \infty} H\left(f_{n}\right)=0$.

Similar to [7], we get the following strict concavity and strict subadditivity of $E_{\lambda}^{d_{\mathrm{av}}}$.

Proposition B.2 (Strict subadditivity). Under assumptions $\boldsymbol{A} 1$ and $\mathbf{A} 2$ and for any $\lambda>0,0<\delta<\lambda / 2$, and $\lambda_{1}, \lambda_{2} \geq \delta$ with $\lambda_{1}+\lambda_{2} \leq \lambda$, we have

$$
E_{\lambda_{1}}^{d_{\mathrm{av}}}+E_{\lambda_{2}}^{d_{\mathrm{av}}} \geq\left[1-\left(2^{\frac{\gamma_{0}}{2}}-2\right)\left(\frac{\delta}{\lambda}\right)^{\frac{\gamma_{0}}{2}}\right] E_{\lambda}^{d_{\mathrm{av}}}
$$

where $\gamma_{0}>2$ as in $\mathbf{A} 2$.

Remark B.3. In particular, Proposition B.2 shows that for any $\lambda_{1}, \lambda_{2}>0$ one has

$$
E_{\lambda_{1}}^{d_{\mathrm{av}}}+E_{\lambda_{2}}^{d_{\mathrm{av}}}>E_{\lambda_{1}+\lambda_{2}}^{d_{\mathrm{av}}}
$$

as soon as $E_{\lambda_{1}+\lambda_{2}}^{d_{\mathrm{av}}}<0$. That is, the map $\lambda \mapsto E_{\lambda}^{d_{\mathrm{av}}}$ is strictly subadditive where it is strictly negative.

In order to prove this, we need a little preparation.

Lemma B.4. $V$ obeys $A 2$ if and only if for all $t \geq 1$ we have

$$
V(t a) \geq t^{\gamma_{0}} V(a) \text { for all } a>0 .
$$

Proof. Assume that $V$ obeys A2. Then

for all $a>0$ and $t>1$. Thus

$$
\frac{d}{d t} V(t a)=V^{\prime}(t a) a \geq \frac{\gamma_{0}}{t} V(t a)
$$

and integrating this yields (B.2).

$$
\frac{d}{d t}\left(t^{-\gamma_{0}} V(t a)\right) \geq 0
$$

Conversely, since (B.2) is an equality for $t=1$, we can differentiate it at $t=1$ to get A2.

Proof of Proposition B.2. Let $t \geq 1$, then A2 and Lemma B.4 imply $N(t f) \geq t^{\gamma_{0}} N(f)$ for any $f \in l^{2}(\mathbb{Z})$. Thus also

$$
H(t f) \leq t^{2} d_{\mathrm{av}}\left\langle D_{+} f, D_{+} f\right\rangle-t^{\gamma_{0}} N(f) \leq t^{\gamma_{0}} H(f)
$$

since $t \geq 1$ and $\gamma_{0}>2$. Hence

$$
s^{\gamma_{0}} H(f) \leq H(s f)
$$

for all $f \in l^{2}(\mathbb{Z})$ and all $0 \leq s \leq 1$ and

$$
s^{\gamma_{0}} E_{\lambda}^{d_{\text {av }}}=s^{\gamma_{0}} \inf _{\|f\|_{2}^{2}=\lambda} H(f) \leq \inf _{\|f\|_{2}^{2}=s^{2} \lambda} H(f)=E_{s^{2} \lambda}^{d_{\text {av }}} .
$$

For $\lambda_{1}, \lambda_{2}>0$ and $\lambda_{1}+\lambda_{2} \leq \lambda$ choose $0<\mu_{j}<1$ with $\lambda_{j}=\mu_{j} \lambda$ for $j=1,2$. Then

$$
E_{\lambda_{1}}^{d_{\mathrm{av}}}+E_{\lambda_{2}}^{d_{\mathrm{av}}}=E_{\mu_{1} \lambda}^{d_{\mathrm{av}}}+E_{\mu_{2} \lambda}^{d_{\mathrm{av}}} \geq\left(\mu_{1}^{\gamma_{0} / 2}+\mu_{2}^{\gamma_{0} / 2}\right) E_{\lambda}^{d_{\mathrm{av}}}
$$

because of (B.4). Without loss of generality we can assume $\mu_{1} \geq \mu_{2}$, otherwise we simply exchange $\lambda_{1}$ and $\lambda_{2}$. Then, since $0<\mu_{1}+\mu_{2} \leq 1$, we have

$$
\begin{aligned}
\mu_{1}^{\gamma_{0} / 2}+\mu_{2}^{\gamma_{0} / 2} & \leq 1-\left(\left(\mu_{1}+\mu_{2}\right)^{\gamma_{0} / 2}-\mu_{1}^{\gamma_{0} / 2}-\mu_{2}^{\gamma_{0} / 2}\right) \\
& =1-\mu_{2}^{\gamma_{0} / 2}\left(\left(1+\frac{\mu_{1}}{\mu_{2}}\right)^{\gamma_{0} / 2}-\left(\frac{\mu_{1}}{\mu_{2}}\right)^{\gamma_{0} / 2}-1\right)
\end{aligned}
$$




$$
\leq 1-\mu_{2}^{\gamma_{0} / 2}\left(2^{\gamma_{0} / 2}-2\right)
$$

where the last inequality follows from the fact that for $\gamma_{0}>2$ the map $0<s \mapsto(1+s)^{\gamma_{0} / 2}-$ $s^{\gamma_{0} / 2}-1$ is increasing on $[1, \infty)$. Thus, since $E_{\lambda}^{d_{\mathrm{av}}} \leq 0$ by Lemma B.1 and $\mu_{2} \geq \delta / \lambda$, the inequality (B.1) follows.

Lemma B.5. Assume that assumption A4 holds and $\lambda>0$. Then $E_{\lambda}^{d_{\mathrm{av}}}<0$.

Proof. Unlike the continuous case, where Gaussians provide a nice class of initial conditions $f$ for which one can explicitly calculate the time evolution $T_{r} f$, no such class of functions exists in the discrete case. Hence, the proof that $E_{\lambda}^{d_{\mathrm{av}}}$ is strictly negative is quite different from the continuous case.

Recall that $E_{\lambda}^{d_{\mathrm{av}}}$ is defined in (1.5). We consider the case $d_{\mathrm{av}}=0$ first. Let $h_{1, p}(r):=$ $e^{4|r|\left|1-\frac{2}{p}\right|}$. Assumption A4 says that if $d_{\mathrm{av}}=0$, then there exists $\varepsilon>0$ such that $V(a)>0$ for all $0<a \leq \varepsilon$. Let $B>0$ such that $\operatorname{supp} \mu \subset[-B, B]$ and for any $\nu>0$ take $f_{\nu}(x)=A_{\nu} e^{-\nu|x|}$ with

$$
A_{\nu}:=\lambda^{1 / 2}\left(\frac{\sinh (\nu)}{\cosh (\nu)}\right)^{1 / 2} .
$$

Then (A.8) from Lemma A.1 shows $\left\|f_{\nu}\right\|_{2}^{2}=\lambda$, i.e., $f_{\nu}$ is a valid test function. Moreover, $A_{\nu}$ is increasing in $\nu$ with $A_{\nu} \rightarrow 0$ as $\nu \rightarrow 0+$ so $\left\|f_{\nu}\right\|_{\infty}=A_{\nu} \leq \varepsilon / h_{1, \infty}(B)$ for all small enough $\nu>0$, hence, because of (A.2), there exists $\nu_{1}>0$ such that $\left\|T_{r} f_{\nu}\right\|_{\infty} \leq \varepsilon$ for all $|r| \leq B$ and $0<\nu \leq \nu_{1}$. In this case, by assumption $\mathbf{A} 4$,

$$
V\left(\left|T_{r} f_{\nu}(x)\right|\right) \geq 0 \quad \text { for all } x \in \mathbb{Z},|r| \leq B, 0<\nu \leq \nu_{1},
$$

hence $N\left(f_{\nu}\right) \geq 0$. If $E_{\lambda}^{0}=0$, we would have $0=E_{\lambda}^{0} \leq-N\left(f_{\nu}\right) \leq 0$, so

$$
N\left(f_{\nu}\right)=\int_{\mathbb{R}} \sum_{x \in \mathbb{Z}} V\left(\left|T_{r} f_{\nu}(x)\right|\right) \mu(d r)=0 .
$$

Because of (B.6), this implies for all $0<\nu \leq \nu_{1}$,

$$
V\left(\left|T_{r} f_{\nu}(x)\right|\right)=0 \quad \text { for } \mu \text {-almost all } r \text { and all } x \in \mathbb{Z} \text {. }
$$

and since $0 \leq\left|T_{r} f_{\nu}(x)\right| \leq \varepsilon$, the only way this can be is if

$$
T_{r} f_{\nu}=0 \text { for } \mu \text {-almost all } r
$$

and since $T_{r}$ is unitary on $l^{2}(\mathbb{Z})$, this implies $f_{\nu}=0$ for all small enough $\nu$, which is a contradiction. Thus $E_{\lambda}^{0}<0$ if $\lambda>0$.

In the case $d_{\mathrm{av}}>0$, A4 shows that there exist $\varepsilon>0$ and $2 \leq \kappa<6$ such that $V(a) \gtrsim a^{\kappa}$ for all $0 \leq a \leq \varepsilon$. Again let $B>0$ such that supp $\mu \subset[-B, B]$ and choose $f_{\nu}(x):=A_{\nu} e^{-\nu|x|}$ with $A_{\nu}$ given by (B.5) and $\nu_{2}>0$ such that $\left\|f_{\nu}\right\|_{\infty}=A_{\nu} \leq \varepsilon / h_{1, \infty}(2 B)$ for all $0<\nu \leq \nu_{2}$. Then the second part of Lemma A.1 guarantees $\left\|T_{r-r_{0}} f_{\nu}\right\|_{\infty} \leq \varepsilon$ for all $r_{0}, r \in \operatorname{supp} \mu$ and $0<\nu \leq \nu_{2}$.

Set $g:=T_{-r_{0}} f_{\nu}$, then $0 \leq\left|T_{r} g\right| \leq \varepsilon$ for all $r \in \operatorname{supp} \mu$, hence

$$
N(g)=\int_{\mathbb{R}} \sum_{x \in \mathbb{Z}} V\left(\left|T_{r} g(x)\right|\right) \mu(d r) \gtrsim \int_{\mathbb{R}} \sum_{x \in \mathbb{Z}}\left|T_{r} g(x)\right|^{\kappa} \mu(d r) \geq \int_{r_{0}-\delta}^{r_{0}+\delta}\left\|T_{r} g\right\|_{\kappa}^{\kappa} \mu(d r)
$$

for all $r_{0} \in \operatorname{supp} \mu$ and any $\delta>0$. Define $h_{2}(r):=e^{4|r|}-1$. Then the bounds from Lemma A.1 give

$$
\left\|T_{r} g\right\|_{\kappa}^{\kappa} \geq\left\|f_{\nu}\right\|_{\kappa}^{\kappa}-\left|\left\|f_{\nu}\right\|_{\kappa}^{\kappa}-\left\|T_{r-r_{0}} f_{\nu}\right\|_{\kappa}^{\kappa}\right|
$$




$$
\begin{aligned}
& \geq\left\|f_{\nu}\right\|_{\kappa}^{\kappa}-\kappa \max \left(\left\|f_{\nu}\right\|_{\kappa}^{\kappa-1},\left\|T_{r-r_{0}} f_{\nu}\right\|_{\kappa}^{\kappa-1}\right)\left\|f_{\nu}-T_{r-r_{0}} f_{\nu}\right\|_{\kappa} \\
& \geq\left(1-\kappa\left(h_{1, \kappa}\left(r-r_{0}\right)\right)^{\kappa-1} h_{2}\left(r-r_{0}\right)\right)\left\|f_{\nu}\right\|_{\kappa}^{\kappa} .
\end{aligned}
$$

Thus

$$
N(g) \gtrsim\left(1-\kappa\left(h_{1, \kappa}(2 B)\right)^{\kappa-1} h_{2}(\delta)\right) \mu\left(\left(r_{0}-\delta, r_{0}+\delta\right)\right)\left\|f_{\nu}\right\|_{\kappa}^{\kappa}
$$

Since the (complement of the) support of the measure $\mu$ is given by

$$
(\operatorname{supp} \mu)^{c}=\left\{r_{0} \in \mathbb{R} \mid \exists \delta>0 \text { such that } \mu\left(\left(r_{0}-\delta, r_{0}+\delta\right)\right)=0\right\}
$$

one has, for any $r_{0} \in \operatorname{supp} \mu$,

$$
\mu\left(\left(r_{0}-\delta, r_{0}+\delta\right)\right)>0 \text { for all } \delta>0 .
$$

So choosing any $r_{0} \in \operatorname{supp} \mu$ and $\delta>0$ small enough that $1-\kappa\left(h_{1, \kappa}(2 B)\right)^{\kappa-1} h_{2}(\delta)>0$ yields

$$
N(g) \gtrsim\left\|f_{\nu}\right\|_{\kappa}^{\kappa}=\lambda^{\kappa / 2}\left(\frac{\sinh (\nu)}{\cosh (\nu)}\right)^{\kappa / 2} \frac{\cosh \left(\frac{\kappa}{2} \nu\right)}{\sinh \left(\frac{\kappa}{2} \nu\right)}
$$

where the implicit constant depends only on $\delta>0$ and the constant in the lower bound on $V$ from assumption A3, in particular, it does not depend on $0<\nu \leq \nu_{2}$.

Since $\Delta$ and $T_{r}$ commute, by (A.8),

$$
\langle g,-\Delta g\rangle=\left\langle f_{\nu},-\Delta f_{\nu}\right\rangle=4 \lambda \frac{\sinh ^{2}(\nu / 2)}{\cosh (\nu)}
$$

and choosing $g:=T_{-r_{0}} f_{\nu}$ as a test function in the energy $H$ shows

$$
\begin{aligned}
E_{\lambda} & \leq H(g)=\frac{d_{\mathrm{av}}}{2}\langle g,-\Delta g\rangle-N(g) \\
& \leq 2 d_{\mathrm{av}} \lambda \frac{\sinh ^{2}(\nu / 2)}{\cosh (\nu)}-C \lambda^{\kappa / 2}\left(\frac{\sinh (\nu)}{\cosh (\nu)}\right)^{\kappa / 2} \frac{\cosh \left(\frac{\kappa}{2} \nu\right)}{\sinh \left(\frac{\kappa}{2} \nu\right)} \\
& =\sinh (\nu / 2)^{2}\left(\frac{d_{\mathrm{av}} \lambda}{\cosh (\nu)}-C \lambda^{\kappa / 2} \frac{\cosh \left(\frac{\kappa}{2} \nu\right)}{\cosh ^{\kappa / 2}(\nu)} \frac{\sinh ^{\kappa / 2}(\nu)}{\sinh \left(\frac{\kappa}{2} \nu\right) \sinh ^{2}(\nu / 2)}\right)
\end{aligned}
$$

As $\nu \rightarrow 0 \sinh (s \nu)=O(\nu)$ and $\cosh (s \nu)=O(1)$ for any fixed $s \neq 0$. So

$$
E_{\lambda} \leq O\left(\nu^{2}\right)\left(1-O\left(\nu^{\frac{\kappa}{2}-3}\right)\right)<0
$$

for small enough $\nu>0$, since $\kappa<6$. This shows that $E_{\lambda}<0$ for all $\lambda>0$.

Lemma B.6. Assume that assumptions A1 through A3 hold. Then there exists $f \in l^{2}(\mathbb{Z})$ such that

$$
N(f)=\int_{\mathbb{R}} \sum_{x \in \mathbb{Z}} V\left(\left|T_{r} f(x)\right|\right) \mu(d r)>0 .
$$

Proof. Let $l \in \mathbb{N}$ and set $u_{l}(r, \cdot):=T_{r} \mathbf{1}_{[-2 l, 2 l]}$. Since $\mu$ is a finite measure with compact support there exits $0<B<\infty$ with supp $\mu \subset[-B, B]$. We claim that for some constant $c>0$ and all large enough $l \in \mathbb{N}$ the bounds

$$
\begin{array}{ll}
\left|u_{l}(r, x)\right|-1 \gtrsim-e^{-c l} & \text { for all }|x| \leq l,|r| \leq B \\
\left|u_{l}(r, x)\right| \lesssim l e^{-c(|x|-2 l)} & \text { for all }|x| \geq 3 l,|r| \leq B
\end{array}
$$

hold. We will prove them later. Assumptions A2 and A3, together with Lemma B.4 show that there exists $a_{0}>0$ such that $V(a) \gtrsim a^{\gamma_{0}}$ for all $a \geq a_{0}$ and using assumption A1, 
we have $V(a) \gtrsim-a^{\gamma_{1}}$ for $0 \leq a<a_{0}$. Thus, with $\gamma:=\min \left(\gamma_{0}, \gamma_{1}\right)$, we see that the lower bound

$$
V(a) \gtrsim-a^{\gamma} \mathbf{1}_{\left[0, a_{0}\right)}(a)+a^{\gamma} \mathbf{1}_{\left[a_{0}, \infty\right)}(a)
$$

holds and $V$ is bounded from below.

By (B.7) we can choose $l$ and $\alpha$ large enough such that $\alpha\left|u_{l}(r, x)\right| \geq \frac{\alpha}{2} \geq a_{0}$ for all $|x| \leq l,|r| \leq B$. Then (B.9) yields

$$
I:=\sum_{|x| \leq l} V\left(\alpha\left|u_{l}(r, x)\right|\right) \gtrsim l \alpha^{\gamma} .
$$

Since $V$ is bounded from below, we also have

$$
I I:=\sum_{l<|x| \leq 3 l} V\left(\alpha\left|u_{l}(r, x)\right|\right) \gtrsim-l
$$

and (B.9) together with (B.8) gives

$$
I I I:=\sum_{|x|>3 l} V\left(\alpha\left|u_{l}(r, x)\right|\right) \gtrsim-(\alpha l)^{\gamma} \sum_{|x|>3 l} e^{-c \gamma(|x|-2 l)} \gtrsim-(\alpha l)^{\gamma} e^{-c \gamma(l+1)}
$$

for all $|r| \leq B$. Thus, since $\mu$ is a finite measure with support in $[-B, B]$, this gives the lower bound

$$
N\left(\alpha \mathbf{1}_{[-2 l, 2 l]}\right) \gtrsim I+I I+I I I \gtrsim l \alpha^{\gamma}-l-(\alpha l)^{\gamma} e^{-c \gamma(l+1)}
$$

for all large enough $\alpha$ and $l$. Setting $\alpha=l$ shows $\lim _{l \rightarrow \infty} N\left(l \mathbf{1}_{[-2 l, 2 l]}\right)=\infty$, in particular, $N(f)>0$ for some $f \in l^{2}(\mathbb{Z})$.

It remains to prove (B.7) and (B.8). From Lemma A.1, more precisely, (A.4), we have the bound $\left|\left\langle x\left|T_{r}\right| y\right\rangle\right| \leq \min \left(1, e^{4|r|} \frac{(4|r|)^{|x-y|}}{|x-y| !}\right)$ for any $r \in \mathbb{R}$. Thus, for all $|r| \leq B$

$$
\left|u_{l}(r, x)\right| \leq \sum_{|y| \leq 2 l}\left|\left\langle x\left|T_{r}\right| y\right\rangle\right| \lesssim \sum_{|y| \leq 2 l} \frac{(4 B)^{|x-y|}}{(|x-y|) !},
$$

The map $n \mapsto \frac{(4 B)^{n}}{n !}$ is decreasing for all $n \geq 4 B-1$. For $|x| \geq 3 l$ and all $|y| \leq 2 l$ we will have $n=|x-y| \geq|x|-2 l \geq 4 B-1$ for all large enough $l$, hence we can replace $|x-y|$ above by $|x|-2 l$ and use $n ! \geq e^{n \ln n-n}$ to arrive at to see

$$
\left|u_{l}(r, x)\right| \lesssim l \frac{(4 B)^{|x|-2 l \mid}}{(|x|-2 l) !} \leq l e^{(1+\ln (4 B)-\ln (|x|-2 l))(|x|-2 l)} \lesssim l e^{-c(|x|-2 l)}
$$

for some constant $c>0$ and all $|x| \geq 3 l$ with $l$ large enough. This proves (B.8).

For any initial condition $f_{0}$, the time evolution $u(r, \cdot)=T_{r} f_{0}$ is given by the convergent series

$$
u(r, x)=\left\langle\delta_{x}, T_{r} f_{0}\right\rangle=f_{0}+\sum_{n=1}^{\infty} \frac{(i r)^{n}}{n !}\left\langle\delta_{x}, \Delta^{n} f_{0}\right\rangle
$$

If $f_{0}=\mathbf{1}_{[-2 l, 2 l]}$, then $\Delta f_{0}=-\delta_{2 l+1}+\delta_{2 l}-\delta_{-(2 l+1)}+\delta_{-2 l}$, where $\delta_{y}$ is the Kronecker delta at $y \in \mathbb{Z}$. Moreover, since $\Delta$ increases the support by at most one, that is, $\min (\operatorname{supp} \Delta f) \geq$ $\min (\operatorname{supp} f)-1$ and $\max (\operatorname{supp} \Delta f) \leq \max (\operatorname{supp} f)+1$, and $\|\Delta f\|_{\infty} \leq 4\|f\|_{\infty}$, we see that for any $1 \leq n \leq 2 l$ there exists $g_{n, l}: \mathbb{Z} \rightarrow \mathbb{R}$ with $\left\|g_{n, l}\right\|_{\infty} \leq 1, \operatorname{supp} g_{n, l} \subset[2 l-n+1,2 l+n]$ and

$$
\left\langle\delta_{x}, \Delta^{n} \mathbf{1}_{[-2 l, 2 l]}\right\rangle=\left(\Delta^{n} \mathbf{1}_{[-2 l, 2 l]}\right)(x)=4^{n}\left(g_{n, l}(x)+g_{n, l}(-x)\right)
$$


for all $x \in \mathbb{Z}$. In particular, $\left\langle\delta_{x}, \Delta^{n} \mathbf{1}_{[-2 l, 2 l]}\right\rangle=0$ for all $|x| \leq l$ and all $1 \leq n \leq l$. So the series for $u_{l}(r, x)$ gives

$$
u_{l}(r, x)=\mathbf{1}_{[-2 l, 2 l]}(x)+\sum_{n \geq l+1} \frac{(i r)^{n}}{n !}\left\langle\delta_{x}, \Delta^{n} \mathbf{1}_{[-2 l, 2 l]}\right\rangle \quad \text { for all }|x| \leq l,
$$

hence by the same calculation as for (A.13)

$$
\left|u_{l}(r, x)\right| \geq 1-\sum_{n \geq l+1} \frac{(4|r|)^{n}}{n !} \geq 1-\frac{e^{4|r|}|4 r|^{l+1}}{(l+1) !} \quad \text { for all }|x| \leq l .
$$

Bounding $(l+1) ! \geq e^{(l+1) \ln (l+1)-(l+1)}$ shows that (B.7) is true for some $c>0$ and all large enough $l \in \mathbb{N}$.

\section{Appendix C. The Discrete IMS localization formula}

Here we give a simple bound which is useful for localizing the discrete kinetic energy.

Lemma C.1. Let $f \in l^{2}(\mathbb{Z})$ and $\xi: \mathbb{Z} \rightarrow \mathbb{R}$ be a bounded function. Then,

$$
\operatorname{Re}\left(\left\langle\xi^{2} f,-\Delta f\right\rangle\right)=\langle\xi f,-\Delta(\xi f)\rangle-\sum_{x \in \mathbb{Z}}\left|D_{+} \xi(x)\right|^{2} \operatorname{Re}(\overline{f(x)} f(x+1)) .
$$

In particular, the lower bound

$$
\operatorname{Re}\left(\left\langle\xi^{2} f,-\Delta f\right\rangle\right) \geq\langle\xi f,-\Delta(\xi f)\rangle-\frac{1}{2}\left\langle f,\left(\left|D_{+} \xi\right|^{2}+\left|D_{-} \xi\right|^{2}\right) f\right\rangle
$$

holds and if $\xi_{j}: \mathbb{Z} \rightarrow \mathbb{R}, j=1, \ldots, n$ are finitely many bounded functions with $\sum_{j=1}^{n} \xi_{j}^{2}=1$, then

$$
\langle f,-\Delta f\rangle \geq \sum_{j=1}^{n}\left\langle\xi_{j} f,-\Delta\left(\xi_{j} f\right)\right\rangle-\frac{1}{2} \sum_{j=1}^{n}\left\langle f,\left(\left|D_{+} \xi_{j}\right|^{2}+\left|D_{-} \xi_{j}\right|^{2}\right) f\right\rangle .
$$

Here $D_{+} \xi(x):=\xi(x+1)-\xi(x)$ and $D_{-} \xi(x):=\xi(x)-\xi(x-1)$ the forward and backward differences.

Proof. A simple commutator calculation shows

$$
[\Delta, \xi]=\left(D_{+} \xi\right) S_{+}-\left(D_{-} \xi\right) S_{-}
$$

where $\left(S_{+} f\right)(x):=f(x+1)$ is the left shift and $\left(S_{-} f\right)(x):=f(x-1)$ is the right shift. Another calculation shows

$$
[[\Delta, \xi], \xi]=\left|D_{+} \xi\right|^{2} S_{+}+\left|D_{-} \xi\right|^{2} S_{-}
$$

and expanding the commutator gives

$$
\xi^{2} \Delta-2 \xi \Delta \xi+\Delta \xi^{2}=[[\Delta, \xi], \xi] .
$$

Thus

$$
\begin{aligned}
2 \operatorname{Re}\left(\left\langle\xi^{2} f,\right.\right. & -\Delta f\rangle)=\left\langle f,-\left(\xi^{2} \Delta+\Delta \xi^{2}\right) f\right\rangle=2\langle\xi f,-\Delta(\xi f)\rangle-\langle f,[[\Delta, \xi], \xi] f\rangle \\
& =2\langle\xi f,-\Delta(\xi f)\rangle-\sum_{x \in \mathbb{Z}}\left(\left|D_{+} \xi(x)\right|^{2} \overline{f(x)} f(x+1)+\left|D_{-} \xi(x)\right|^{2} f(x-1) \overline{f(x)}\right) \\
& =2\langle\xi f,-\Delta(\xi f)\rangle-\sum_{x \in \mathbb{Z}}\left|D_{+} \xi(x)\right|^{2} 2 \operatorname{Re}(\overline{f(x)} f(x+1)),
\end{aligned}
$$


since $D_{-} \xi(x+1)=D_{+} \xi(x)$, which proves (C.1). The bound (C.2) follows from (C.1) since

$$
\begin{aligned}
\sum_{x \in \mathbb{Z}}\left|D_{+} \xi(x)\right|^{2} \operatorname{Re}(\overline{f(x)} f(x+1)) & \leq \frac{1}{2} \sum_{x \in \mathbb{Z}}\left|D_{+} \xi(x)\right|^{2}\left(|f(x)|^{2}+|f(x+1)|^{2}\right) \\
& =\frac{1}{2} \sum_{x \in \mathbb{Z}}\left(\left|D_{+} \xi(x)\right|^{2}+\left|D_{-} \xi(x)\right|^{2}\right)|f(x)|^{2}
\end{aligned}
$$

Moreover, if $\sum_{j} \xi_{j}^{2}=1$, then

$$
\langle f,-\Delta f\rangle=\operatorname{Re}(\langle f,-\Delta f\rangle)=\sum_{j=1}^{n} \operatorname{Re}\left(\left\langle\xi_{j}^{2} f,-\Delta f\right\rangle\right)
$$

so (C.3) follows from (C.2).

\section{Appendix D. The CONnECTION With NONLINEAR OPTICS}

Our main motivation for studying (1.1) and the related minimization problems (1.5) comes from the fact that the solutions are related to breather-type solutions of the diffraction managed discrete nonlinear Schrödinger equation

$$
i \partial_{t} u=-d(t) \Delta u-P(u),
$$

where $\Delta$ is the nearest neighbour discrete Laplacian, $t$ the distance along the waveguide, $x \in \mathbb{Z}$ the location of the waveguide, $d(t)$ the local diffraction along the waveguide, and $P(u)$ is an on site nonlinear interaction. This equation describes, for example, an array of coupled nonlinear waveguides $[4,5,11,21,28]$, but it also models a wide range of effects ranging from molecular crystals $[6,26]$ to biophysical systems $[9,10]$. By symmetry, one assumes that $P$ is odd and $P(0)=0$ can always be enforced by adding a constant term. Most often one makes a Taylor series expansion, keeping just the lowest order nontrivial term leads to $P(u) \simeq|u|^{2} u$, the Kerr nonlinearity, but we will not make this approximation. The study of bound states of the discrete nonlinear Schrödinger equation (D.1) has attraction a lot of attention, see, for example, [17] and the references therein.

The idea to periodically alter the diffraction along the waveguide by creating a zigzag geometry of the waveguides, similar to what has been done in dispersion management cables, see, for example, $[13,27,30]$ and the references therein, was probably first conceived in [12] in order to create low power stable discrete pulses. In this case, the total diffraction $d(t)$ along the waveguide is given by

$$
d(t)=\varepsilon^{-1} d_{0}(t / \varepsilon)+d_{\mathrm{av}} .
$$

Here $d_{\mathrm{av}}$ is the average component of the diffraction and $d_{0}$ its periodic mean zero part with period $L$.

A technical complication is the fact that (D.1) is a non-autonomous equation. We seek to rewrite (D.1) into a more convenient form in order to find breather type solutions. In the region of strong diffraction management $\varepsilon$ is a small positive parameter. In this parameter region an average equation which describes the evolution of the slow part of solutions of (D.1) was derived in Fourier space in [1, 2, 3], using the same general method as in the continuum case, see, e.g., [30]. The numerical studies of $[1,2,3]$ showed that this average equation possesses stable solutions which evolve nearly periodically when used as initial data in the diffraction managed non-linear discrete Schrödinger equation. To derive this equation in our notation, let $T_{r}:=e^{-i r \Delta}$ be the free discrete Schrödinger evolution, set

$$
D(s):=\int_{0}^{s} d_{0}(\zeta) d \zeta
$$


and make the ansatz

$$
u(t, x)=T_{D\left(\frac{t}{\varepsilon}\right)} v
$$

for some function $v$. Then, since $\partial_{t} T_{D\left(\frac{t}{\varepsilon}\right)}=\frac{1}{\varepsilon} d_{0}\left(\frac{t}{\varepsilon}\right) \Delta T_{D\left(\frac{t}{\varepsilon}\right)}$, we get from (D.1) and (D.2) that $v$ solves

$$
i \partial_{t} v(t, x)=-d_{\mathrm{av}} \Delta v(t, x)-T_{D\left(\frac{t}{\varepsilon}\right)}^{-1}\left[P\left(T_{D\left(\frac{t}{\varepsilon}\right)} v(t, \cdot)\right)\right](x)
$$

for $t \geq 0$ and $x \in \mathbb{Z}$, which is equivalent to (D.1) and still a non-autonomous equation. Since $d_{0}$ has average zero and period $L, D$ is periodic with the same period $L$ and thus for small $\varepsilon>0$ the function $t \mapsto D\left(\frac{t}{\varepsilon}\right)$ is highly oscillatory with period $\varepsilon L$. Similar to Kapitza's treatment of the stabilization of the unstable pendulum by high frequency oscillations of the pivot, see [18], the evolution of $v$ should evolve on two different scales, a slow one plus a high frequency one with a small amplitude. The evolution of the slow part $v_{\text {slow }}$ is described by an averaged equation, where one averages over the fast oscillating terms,

$$
\begin{aligned}
i \partial_{t} v_{\text {slow }}(t, x) & =-d_{\text {av }} \Delta v_{\text {slow }}(t, x)-\frac{1}{\varepsilon L} \int_{0}^{\varepsilon L} T_{D\left(\frac{s}{\varepsilon}\right)}^{-1}\left[P\left(T_{D\left(\frac{s}{\varepsilon}\right)} v_{\text {slow }}(t, \cdot)\right)\right](x) d s \\
& =-d_{\text {av }} \Delta v_{\text {slow }}(t, x)-\frac{1}{L} \int_{0}^{L} T_{D(s)}^{-1}\left[P\left(T_{D(s)} v_{\text {slow }}(t, \cdot)\right)\right](x) d s .
\end{aligned}
$$

Making the substitution $r=D(s)$ and introducing the probability measure $\mu$ on $\mathbb{R}$ defined by

$$
\int_{\mathbb{R}} F(r) \mu(d r):=\frac{1}{L} \int_{0}^{L} F(D(s)) d s
$$

for any nonnegative (Borel) measurable functions $F$, one has

$$
i \partial_{t} v_{\text {slow }}(t, x)=-d_{\text {av }} \Delta v_{\text {slow }}(t, x)-\int_{\mathbb{R}} T_{r}^{-1}\left[P\left(T_{r} v_{\text {slow }}(t, \cdot)\right)\right](x) \mu(d r)
$$

which is the time dependent version of (1.1). To derive (1.1) from it, one simply makes the ansatz $v_{\text {slow }}(t, x)=e^{i \omega t} \varphi(x)$, to see that this solves (D.4) if and only if $\varphi$ solves (1.1).

Physically it makes sense to assume that the diffraction profile $d_{0}$ is bounded, or even piecewise constant along the waveguide, but one might envision much more complicated scenarios. The simplest case of dispersion management, $L=2, d_{0}=1$ on $[0,1)$ and $d_{0}=-1$ on $[1,2)$, i.e., $d_{0}=\mathbf{1}_{[0,1)}-\mathbf{1}_{[1,2)}$, which is the case most studied in the literature, correspond to a very simple zigzag geometry of the waveguides, $[1,2,3]$. In this case, the measure $\mu$ is very simple, having density $\mathbf{1}_{[0,1]}$, the uniform distribution on $[0,1]$, with respect to Lebesgue measure. This assumption was made in [20, 23, 25], where equation (1.1) was studied for the Kerr type nonlinearity $P(a)=|a|^{2} a$ and also some pure power type modifications thereof in [20].

For our results, which also hold for a much larger class of nonlinearities $P$, we need only to assume the much weaker condition that the probability measure $\mu$ has bounded support, i.e., there exists $B>0$ such that

$$
\mu\left([-B, B]^{c}\right)=\mu((-\infty,-B))+\mu((B, \infty))=0 .
$$

The support condition (D.5) is guaranteed if $d_{0}$ is locally integrable, in which case one take

$$
B:=\sup _{r \in[0, L]}|D(r)| \leq \int_{0}^{L}\left|d_{0}(\xi)\right| d \xi<\infty .
$$

Clearly, this is a very weak assumption on the diffraction profile $d_{0}$ and it has to be assumed in order to even make sense out of equation (D.1). Thus our results cover the most general 
physically allowed local diffraction profiles $d_{0}$, the singular case $d_{0}=0$ leading to the usual discrete NLS which is even local, and cover a large class of nonlinearities $P$.

Acknowledgements: Mi-Ran Choi and Young-Ran Lee thank the Department of Mathematics at KIT and Dirk Hundertmark thanks the Department of Mathematics at Sogang University for their warm hospitality. Dirk Hundertmark gratefully acknowledges financial support by the Deutsche Forschungsgemeinschaft (DFG) through CRC 1173. He also thanks the Alfried Krupp von Bohlen und Halbach Foundation for financial support. Young-Ran Lee thanks the National Research Foundation of Korea(NRF) for financial support funded by the Korea government(MOE) under grant No. 2014R1A1A2058848. We would also like to thank an eclectic array of coffee shops in Seoul and Karlsruhe for providing us with much needed undisturbed time and lots of coffee.

\section{REFERENCES}

[1] M. Ablowitz and Z. H. Musslimani, Discrete Diffraction Managed Spatial Solitons. Phys. Rev. Lett. 87 (2001), 254102 [4 pages].

[2] M. Ablowitz and Z. H. Musslimani, Discrete vector spatial solitons in a nonlinear waveguide array. Phys. Rev. E 65 (2002), 056618 [13 pages].

[3] M. Ablowitz and Z. H. Musslimani, Discrete spatial solitons in a diffraction managed nonlinear waveguide array: a unified approach. Physica D 184 (2003), 276-303.

[4] A. B. Aceves, C. De Angelis, A. M. Rubenchik, and S. K. Turitsyn, Multidimensional solitons in fiber arrays. Opt. Lett. 19 (1994) 32931

[5] A. B. Aceves, C. De Angelis, G.G.G. Luther, and A. M. Rubenchik, Modulational instability of continuous waves and one-dimensional temporal solitons in fiber arrays. Opt. Lett. 19 (1994) 11868

[6] A. S. Barker Jr and A. J. Sievers, Optical studies of the vibrational properties of disordered solids. Rev. Mod. Phys. 47 (1975) S1S179.

[7] M.-R. Choi, D. Hundertmark and Y.-R. Lee, Existence of dispersion management solitons for general nonlinearities, Preprint, 2015. (Available at http://arxiv.org/abs/1508.05888)

[8] H. L. Cycon, R. G. Froese, W. Kirsch and B. Simon, Schrödinger Operators: With Applications to Quantum Mechanics and Global Geometry. Texts and Monographs in Physics, Theoretical and Mathematical Physics, Springer Study Edition. Springer, 2008.

[9] A. S. Davydov, Theory of contraction of proteins under their excitation. J. Theor. Biol. 38 (1973), 559-569.

[10] J. C. Eilbeck, P. S. Lomdahl, and A. C. Scott, The discrete self-trapping equation. Physica D 16 (1985) 318338.

[11] H. Eisenberg, Y. Silverberg, R. Morandotti, A. Boyd and J. Aitchison, Discrete spatial optical solitons in waveguide arrays. Phys. Rev. Lett. 81 (1998), 3383-3386.

[12] H. Eisenberg, Y. Silverberg, R. Morandotti and J. Aitchison, Diffraction management. Phys. Rev. Lett. 85 (2000), 1863-1866.

[13] I. Gabitov and S. K. Turitsyn, Breathing solitons in optical fiber links. JETP Lett. 63 (1996), 861.

[14] D. Hundertmark and Y.-R. Lee, Decay estimates and smoothness for solutions of the dispersion managed non-linear Schrödinger equation, Comm. Math. Phys. 286 (2009), 851-873.

[15] D. Hundertmark and Y.-R. Lee, On non-local variational problems with lack of compactness related to non-linear optics, J. Nonlinear Sci. 22 (2012), 1-38.

[16] D. Hundertmark and Y.-R. Lee, Super-Exponential Decay of Diffraction Managed Solitons, Comm. Math. Phys. 309 (2012), no. 1, 1-21.

[17] M. Jenkinson and M. I. Weinstein, Onsite and offsite bound states of the discrete nonlinear Schrödinger equation and the Peierls-Nabarro barrier. Nonlinearity 29 (2016), 27-86.

[18] L. D. Landau and E. M. Lifshitz, Course of theoretical physics. Vol. 1. Mechanics. Third edition. Pergamon Press, Oxford-New York-Toronto, Ont., 1976. 
[19] R. MacKay, G. Schneider, and D. Pelinovsky, Justification of the lattice equation for a nonlinear elliptic problem with a periodic potential. Commun. Math. Phys. 284 (2008) 803831.

[20] J. Moeser, Diffraction managed solitons: asymptotic validity and excitation thresholds, Nonlinearity 18 (2005), 2275-2297.

[21] R. Morandotti, U. Peschel, J. Aitchison, H. Eisenberg and Y. Silberberg, Dynamics of discrete solitons in optical waveguide array, Phys. Rev. Lett. 83 (1999), 2726-2729.

[22] D. Pelinovsky and G. Schneider, Bounds on the tight-binding approximation for the Gross Pitaevskii equation with a periodic potential. J. Differ. Equ. 248 (2010) 837849.

[23] P. Panayotaros, Breather solutions in the diffraction managed NLS equation. Physica D 206 (2005), 213-231.

[24] W. P. Su, J. R. Schieffer and A. J. Heeger, Solitons in polyacetylene, Phys. Rev. Lett. 42 (1979), 1698-1701.

[25] M. Stanislavova, Diffraction Managed Solitons with Zero Mean Diffraction Journal of Dynamics and Differential Equations 19 (2007), no. 2., 295-307.

[26] S. Takeno, K. Kisoda, and A. J. Sievers, Intrinsic localized vibrational modes in anharmonic crystals. Prog. Theor. Phys. Suppl. 94 (1988) 242269.

[27] S. K. Turitsyn, E. G. Shapiro, S. B. Medvedev, M. P. Fedoruk and V. K. Mezentsev, Physics and mathematics of dispersion-managed optical solitons, Comptes Rendus Physique, Académie des sciences/Éditions scientifiques et médicales 4 (2003), 145-161.

[28] M. I. Weinstein and A. Yeary, Excitation and dynamics of pulses in coupled fiber arrays. Phys. Lett. A 222 (1996) 157-162.

[29] M. I. Weinstein, Excitation thresholds for nonlinear localized modes on lattices, Nonlinearity 12 (1999), 673-691.

[30] V. Zharnitsky, E. Grenier, C. K. R. T. Jones and S. K. Turitsyn, Stabilizing effects of dispersion management, Physica D. 152-153 (2001), 794-817.

Department of Mathematics, Sogang University, 35 Baekbeom-ro (sinsu-dong), Mapo-gu, SeOul, 121-742, South Korea.

E-mail address: rani9030@sogang.ac.kr

Department of Mathematics, Institute for Analysis, Karlsruhe Institute of Technology, 76128 Karlsruhe, Germany.

E-mail address: dirk.hundertmark@kit.edu

Department of Mathematics, Sogang University, 35 Baekbeom-ro (Sinsu-dong), Mapo-Gu, SeOul, 121-742, South Korea.

E-mail address: younglee@sogang.ac.kr 